\title{
Nationwide Evaluation of Urban Energy System Resilience in China Using a Comprehensive Index Method
}

\author{
Ziyi Wang $\left.{ }^{1}{ }^{(}\right)$, Zengqiao Chen ${ }^{2}$, Cuiping Ma ${ }^{1}$, Ronald Wennersten ${ }^{1}$ and Qie Sun ${ }^{3, *}$ \\ 1 Institute of Thermal Science and Technology, Shandong University, Jinan 250061, China; \\ ziyiw@kth.se (Z.W.); macp@sdu.edu.cn (C.M.); rw@kth.se (R.W.) \\ 2 School of Energy and Power Engineering, Shandong University, Jinan 250061, China; czq@sdu.edu.cn \\ 3 Institute for Advanced Technology, Shandong University, Jinan 250061, China \\ * Correspondence: qie@sdu.edu.cn; Tel.: +86-(0)531-8839-2009-308
}

\section{check for} updates

Citation: Wang, Z.; Chen, Z.; Ma, C.; Wennersten, R.; Sun, Q. Nationwide Evaluation of Urban Energy System Resilience in China Using a Comprehensive Index Method. Sustainability 2022, 14, 2077. https:// doi.org/10.3390/su14042077

Academic Editor: Francesco Tajani

Received: 20 December 2021

Accepted: 7 February 2022

Published: 11 February 2022

Publisher's Note: MDPI stays neutral with regard to jurisdictional claims in published maps and institutional affiliations.

Copyright: (C) 2022 by the authors. Licensee MDPI, Basel, Switzerland. This article is an open access article distributed under the terms and conditions of the Creative Commons Attribution (CC BY) license (https:// creativecommons.org/licenses/by/ $4.0 /)$.

\begin{abstract}
The carbon peak and carbon neutrality goals for China signify a critical time of energy transition in which energy resilience is a vital issue. Therefore, a comprehensive evaluation of urban energy system resilience (UESR) is important for establishing a theoretical foundation. To this end, in this paper, 309 Chinese cities were evaluated using a comprehensive UESR assessment framework composed of 113 indices that measured vulnerability and capabilities of resistance and restoration. The results showed that China's UESR is distributed unevenly and that cities in the eastern region generally have higher resilience than those in other regions. The minimum and maximum UESR results corresponded to Tibet and Shandong, respectively, at the provincial level and Rikaze and Weifang, respectively, at the city level. Regression analysis showed a positive correlation among UESR, carbon dioxide emissions, and GDP.
\end{abstract}

Keywords: urban energy system resilience; comprehensive index method; resilience evaluation

\section{Introduction}

On 22 September 2020, President Xi Jinping announced that China would adopt more forceful policies and measures to reach the peak of carbon dioxide emissions by 2030 and to achieve carbon neutrality by 2060; these goals are referred to as the 3060 targets [1]. Energy structure transformation is key to achieving the 3060 targets. The main approaches include reducing the proportion and total amount of fossil fuel consumption, developing renewable energy, reforming the power system, and developing clean and green industries. These approaches assist in building resilient energy systems, as energy system resilience refers to the ability to maintain the essential functions and services of the energy system, ensure stable energy supply and demand with controllable fluctuations, and quickly adapt to new conditions when disruption occurs. Therefore, the 3060 targets, which involve all aspects of energy production, transmission, distribution, consumption, and storage, provide an important opportunity to enhance energy system resilience.

Cities are the macroscopic consumption unit of national energy systems and are responsible for $70 \%$ of global greenhouse gas emissions; thus, they should play an important role in this energy transition [2]. When cities meet various urban energy demands related to citizens' daily lives and provide other infrastructures with enabling functions, a plethora of threats with natural, technical, or human causes might jeopardize the security of their energy systems, leading people to realize that urban energy system resilience (UESR) is becoming increasingly important in the process of urban development [3-5].

Billions of dollars in resilience investment are being mobilized globally, creating demand for a rigorous and decision-oriented resilience measurement [6]. However, the evaluation of UESR has not received much attention or research despite its importance. On the one hand, current research on the evaluation of urban resilience has mainly addressed disturbances due to climate change and natural disasters on cities $[7,8]$, while 
UESR has been rarely studied. As a means of evaluation, the comprehensive index method has been applied to evaluate resilience at the community [6-9], region [10], city [11-13], and country $[14,15]$ levels. For example, resilient city research for China has proposed a set of indicators such as networks and transportation $[9,10]$. However, the energy sector is usually not considered the major focus of urban resilience [9-13]. On the other hand, though energy system resilience has been defined by many researchers [14-20], and the quantification thereof is an important branch of energy system resilience research, there is still no consensus on a suitable and comparable evaluation methodology, and the mainstream quantitative methods have limitations of broad applicability and comparability for various cities. Apart from comprehensive index methods, [21] divided the evaluation methods into two categories: quantitative and qualitative. The quantitative methods are mainly time-dependent matric methods and consider resilience to be capacities of resistance, absorption, and restoration [22-24]. The metrics assess the system performance, which is ad hoc, i.e., system- or event-specific and backed by historical data [25-28]. The complexity and computability of the models and the requirement for historical data limit the broad applicability and comparability of these methods, especially across hundreds of cities. Besides, very few such qualitative methods have been applied to study at the city level. Though a dynamic energy balance-based model has been proposed to measure UESR, this methodology also requires input data and cannot sufficiently providing resilience enhancement strategies at the regional and national levels [29]. Qualitative methods have been less studied; these mainly include checklists and questionnaires [30], the matrix scoring system [31], and the analytic hierarchy process [32]. Case studies to verify feasibility are few as well. In summary, a broadly applicable and comparable quantitative method for evaluating energy system resilience of various cities has not hitherto existed.

To fill this knowledge gap, in this paper, a comprehensive index method is proposed to semi-quantitatively evaluate baseline UESR, which involves the capacities of resistance and restoration combined with vulnerability assessment. To do so, the system boundary of the urban energy system was clarified and UESR was defined; based on the definition, the capacities of resistance and restoration were qualitatively evaluated by three dimensions, namely the multifarious capabilities of the energy system within a city (CE), the interdependencies between other basic city subsystems and the energy system (CI), and the comprehensive vulnerabilities of cities and energy (CV); and these three dimensions were quantitively evaluated by 113 indices, which were selected through a relatively thorough literature review under a set of selection principles. The applicability and comparability of the comprehensive index method are demonstrated through case studies of 309 cities in China.

\section{Materials and Methods}

The resilience discussion herein is proposed to be constrained to high-impact rare events (HR events), also called black swan events [4,33]. The system boundary is constrained on the city level, which represents an adequate unit for policy implementation and is convenient for the overall management of practical events in terms of China's existing realities.

\subsection{Characterization of Urban Energy System (UES)}

The system boundary for an UES can be clarified, as in the working paper of the cross-center UKERC Energy 2050 project [17]. The energy resources, energy carriers, energy technologies, energy infrastructures (physical and virtual), and surrounding supporting facilities in a city are collectively referred to as the UES. Energy resources include fuels, such as coal, charcoal, gasoline, diesel, natural gas, biogas, uranium, and hydrogen, and natural energy sources, such as hydropower, geothermal power, solar power, and wind power. Energy carriers work in terms of electricity, heat, and cold in addition to fuels. Energy technologies are related to centralized power plants, distributed energy systems, and (micro)grids. Supporting facilities incorporate monitoring and protection devices, electric 
energy storage supporting equipment, etc. Generally, the UES can also be traced through the energy flow through production, transmission, distribution, conversion, consumption, and storage within a city's physical boundaries, while part of production, i.e., exploration, exploitation, transportation, and processing, usually occurs outside the UES.

\subsection{Definition of UESR}

In accordance with the essence of the definitions, UESR can be defined as the ability of a UES to resist HR events' impacts, so as to maintain essential functions and services and ensure energy supply and demand within controllable fluctuations, and to quickly restore full energy production. With higher UESR, a UES has a greater capacity to handle foreseeable and/or unforeseeable impacts. From the time dimension, UESR requires the UES to reduce the probability of risk occurrence through measures of risk mitigation in the pre-event stage; diminish the direct and indirect impacts and shorten the duration when an HR event occurs; and withstand various sequential impacts, accommodate and recover from degradation, adapt to new conditions, and learn lessons for future mitigation strategies in the post event stage. In short, for UESs, resilience signifies the capacities of resistance and restoration.

When an HR event occurs, higher resistance helps the UES suffer less performance decline, and higher restoration helps the UES undergo quicker adaptation to new conditions, as shown in Figure 1. The height of the blue-shaded triangle is negatively related to resistance capacity, representing the decrease in system performance. The base of the blueshaded triangle is negatively related to restoration capacity, representing the restoration of the system performance. As the reverse of the blue-shaded area depicts the simplified resilience level, resilience can be determined as follows:

$$
\text { Resilience }=\text { Resistance } \times \text { Restoration }
$$

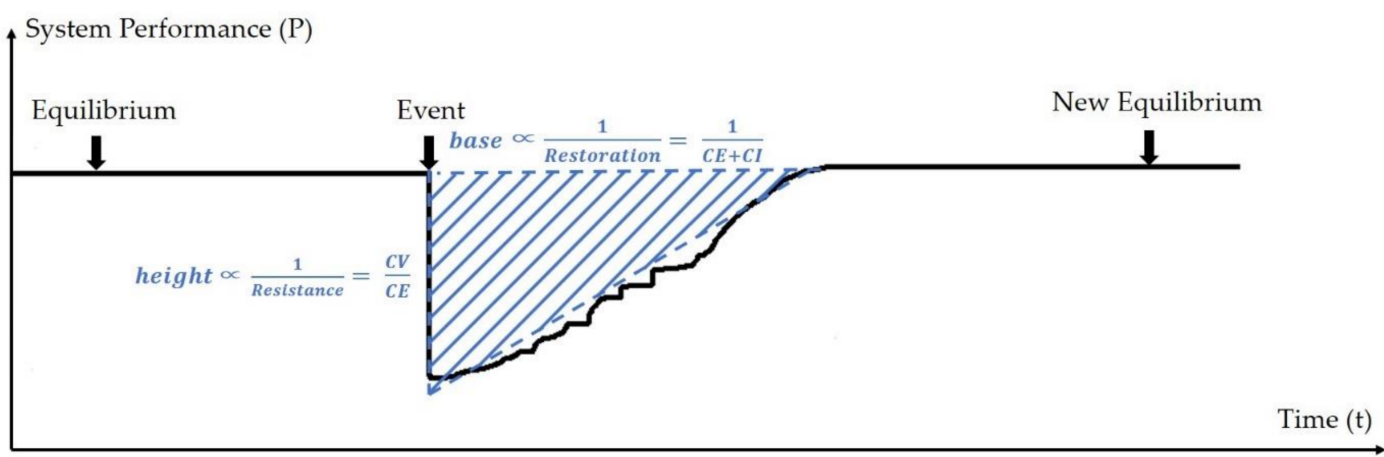

Figure 1. Time-based system performance in an HR event.

To evaluate the capacities of resistance and restoration, three dimensions are proposed: $\mathrm{CE}, \mathrm{CI}$, and CV. CE refers to the comprehensive quality of UESs, including robustness, diversity, flexibility, and availability: (1) robustness refers to the condition of hardware and its ability to resist external impacts to reduce the physical influence of disasters and prevent widespread grid outages and energy supply failures. Hardware refers to grid lines, transformers, energy practitioners, and power generation capacity in this framework. Energy reserves of various fuels play an important role in energy feedstock cutoff. Technological and financial feasibilities should also be considered, e.g., improving energy supply stability and enriching the fuel stock. (2) Diversity consists of energy generation and consumption as well as enterprise productive capacity. To evaluate energy diversity, the Shannon-Weaver index is applied, since it is widely preferred for variety and balance [34]. The Shannon-Weaver index is defined as [35,36]:

$$
D=-\sum_{i} p_{i} \ln \left(p_{i}\right)
$$


where $p_{i}$ represents the share of energy source $i$ in the mix of energy generation/consumption for an energy system. The higher the value of $D$ is, the more diverse a system is evaluated to be. (3) Flexibility is based primarily on the view of the UES as a complex and flexible integrated system that includes organizational, technical, and administrative factors. The system should have the ability to take precautions, study disaster prediction, and obtain the latest information before an event so that rational planning and allocation can be performed in advance in terms of equipment, technology, organization, personnel, resources, and capital. This quality enables the system to flexibly adapt to new internal and external conditions and find a new stable state when an HR event is about to end or after a long period of time following the event. Thus, many aspects at the system-management level are inspected. Evaluation of practice includes demonstration projects, energy savings, and equipment decommission. (4) Availability refers to the ability to adjust the system based on resource availability and financial feasibility. Resource exploitation and processing are considered for coal, petroleum, and other fuels. Financial feasibility is evaluated in terms of the fixed and current assets of energy industries.

$\mathrm{CI}$ involves basic city subsystems that closely interact with the energy system. The interdependencies between critical infrastructures should be taken into consideration since a powerful countermeasure of energy sector that does not explore potential synergies between other pertinent sectors may exacerbate the vulnerability or reduce the overall UESR [37-39]. Thus, CI refers to the capability of a city to cope with hazardous events, including interdependencies between UESs and other societal sectors, such as water, transportation, ecology, emergency services, medical services, and information and telecommunications [40,41]. Water systems are critical in an emergency, and they interact with energy systems via water flow, sewage discharge, cooling water, and circulating water. The transportation system is powered mainly by gasoline, diesel, natural gas and electricity; moreover, the accessibility of the transportation system plays a key role in emergency situations. Ecological systems can provide effective buffering, such as vegetation management and green open space [42]. Emergency services, medical services, and information and telecommunications are high priorities for energy supply and are essential for urban system restoration [43,44].

$\mathrm{CV}$ refers to the number of objects with regard to the basic urban conditions in the city and the energy infrastructures in the energy system, that could possibly be affected by hazard [45-47]. City vulnerability takes demographic, economic, and architectural factors into consideration. Energy vulnerability is associated mainly with pipeline and gas stations of various fuels. District heat and electricity consumption have direct impacts on urban residents' daily lives when HR events occur.

According to the above, the greater the $\mathrm{CE}$ or $\mathrm{CI}$, the faster the system performance is restored; the greater the $\mathrm{CE}$ or the smaller the $\mathrm{CV}$, the less the system performance decreases. The evaluation of resilience, i.e., the UES's capacities of resistance and restoration, is converted into the evaluation of $\mathrm{CE}, \mathrm{CI}$, and $\mathrm{CV}$ as shown in Figure 1 [48].

\subsection{Index Selection}

Comprehensive index methods have become a standard approach to simplifying governmental and organizational policy making, decision making, performance appraisal, and progress tracking at all levels [48]. This study proposes a comprehensive index method, providing each dimension with a series of indices for evaluation. In the early stage of developing the comprehensive index framework, a large number of proposed indices by other researchers and database were collected based on a literature review and data research. The index selection procedure is depicted in Figure 2. To organize a consistent UESR framework, indices must first suit the scope of UES. To this end, hundreds of primary indices were obtained. These primary indices were then classified according to the meaning and category into three dimensions: $\mathrm{CE}, \mathrm{CI}$, and $\mathrm{CV}$. Each index was described in accordance with the referred literature as closely as possible. Following that, a set of selection principles was examined to evaluate the index's systematism, unicity, feasibility, objectivity, and representation. To describe the overall dimension, the index set should 
systematically reflect every subsystem and be neither too detailed nor too general [49]. Unicity means that repeated indices should be removed. Feasibility refers to the availability of data from reliable sources with no obvious errors and the operability of quantitative methods and statistical approaches. To be objective, indices should conform to objective facts and not be interfered with subjective values. Representation means that limited indices should describe a dimension as comprehensively as possible. Indices that met the five selection principles were retained, and those that did not meet any principle were deleted. Detailed primary index selection records are shown in Tables A1-A3 (Appendix A). The deletion of each index was related to its original meaning as it underwent the index selection process. There were two main reasons for deleting indices. Unicity is part of the reason, as most scholars generally attach great importance to output of renewable energy, application of distributed energy system, energy sources, energy diversity, etc. Feasibility was the main reason, because some indices were difficult to quantify, some were not suitable for too many measurement objects because the quantization process was too tedious or the quantization workload was large, and some did not apply to China's actual situation. Therefore, 113 indices were finally retained for the UESR assessment index framework, as shown in Figure 3.

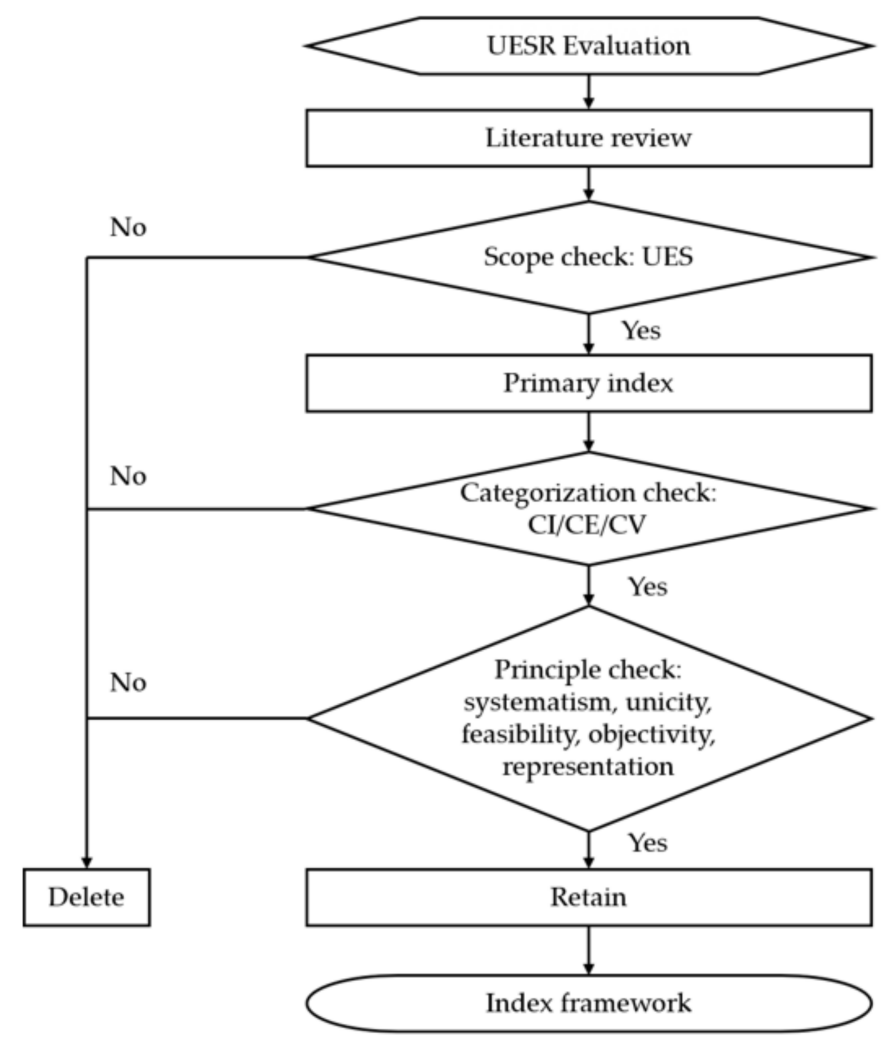

Figure 2. Index selection procedure for UESR evaluation.

The selected 113 indices are quantitatively measured and equally weighted, and they can be assigned differently to satisfy various assessment purposes through a dialogue process between decision makers and stakeholders.

\subsection{Normalization of the Indices and Calculation of UESR}

Indicators were divided into positive and negative indicators according to their supporting or inhibiting effects on resilience [50]. The higher the negative indicators, the lower the corresponding criteria and resilience, such as the share of imported electricity, daily water consumption per capita, and railway access index. All other indicators are positive. Min-max normalization is used to process the original data as follows. 


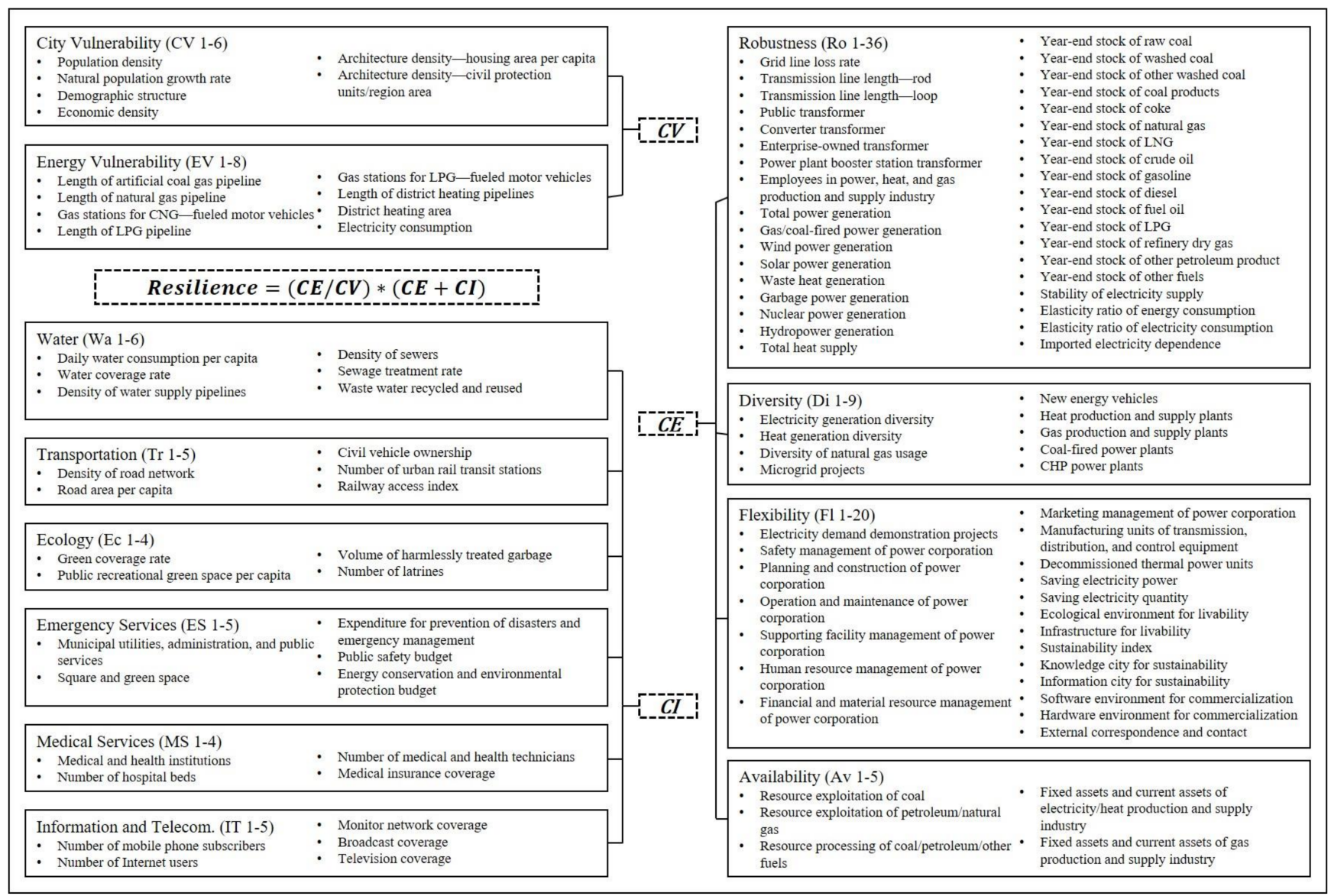

Figure 3. Assessment index for resilience of urban energy systems.

For positive indicators:

$$
y_{i j}=\frac{x_{i j}-\min \left(x_{i j}\right)}{\max \left(x_{i j}\right)-\min \left(x_{i j}\right)}
$$

For negative indicators:

$$
y_{i j}=\frac{\max \left(x_{i j}\right)-x_{i j}}{\max \left(x_{i j}\right)-\min \left(x_{i j}\right)}
$$

where $x_{i j}, y_{i j}$ represent the original and normalized data, respectively; $\max \left(x_{i j}\right)$ is the maximum value of this indicator; and $\min \left(x_{i j}\right)$ is the minimum value of this indicator;

$$
\begin{gathered}
C I=\sum_{i=1}^{n} I_{i} \times \omega_{i} \\
C E=\sum_{i=1}^{n} E_{i} \times \omega_{i}
\end{gathered}
$$

where $I_{i}$ and $E_{i}$ represent the normalized value of index $i$ for $C I$ and $C E$, respectively, and $\omega_{i}$ represents the weight of index $i$. According to the universal risk evaluation model, CV is determined as follows [47]:

$$
V^{2}=\sum_{i=1}^{n} V_{i} \times \omega_{i}
$$


where $V_{i}$ represents the normalized value of index $i$ for city vulnerability or energy vulnerability. Then, resilience is determined as:

$$
\text { Resilience }=\frac{\left(\sum_{i=1}^{n} E_{i} \times \omega_{i}\right) \times\left(\sum_{i=1}^{n} I_{i} \times \omega_{i}+\sum_{i=1}^{n} E_{i} \times \omega_{i}\right)}{\left(\sum_{i=1}^{n} V_{i} \times \omega_{i}\right)^{\frac{1}{2}}}
$$

Based on data survey, statistics, and analysis, the UESR of a city can be obtained by substituting these 113 parameters into Equation (8).

\section{Results}

The energy resilience of 309 Chinese cities is shown in Figure 4. The entire country was divided into four regions according to the National Bureau of Statistics of China [51], namely, the western region (107 cities), the central region (81 cities), the eastern region (87 cities), and the northeastern region (34 cities). Several cities were more resilient than the surrounding areas. There were four types for different reasons. First, provincial capital cities generally had better political resources, management levels, and economic development advantages compared with their surrounding cities and thus had stronger comprehensive city strength and better performance in CI and CE. This applied to Changchun of Jilin, Harbin of Heilongjiang, Taiyuan of Shanxi, Kunming of Yunnan, and Fuzhou of Fujian. Second, Zhangjiakou of Hebei is close to the capital, Beijing, and serves as an important satellite city. It is located in the coal transport corridor, has abundant wind energy resources, has developed a number of microgrid projects, and has few energy-consuming industries, all of which made it a relatively energy-resilient city. Third, Zhuhai of Guangdong has relatively small population density, industrial density, and economic size in Guangdong province, resulting in low CV. As CE and CI were not significantly different, Zhuhai's resilience value was higher. Fourth, Shenzhen of Guangdong was more resilient within the province because of its better performance in energy diversity, microgrid projects, and development of nuclear power.

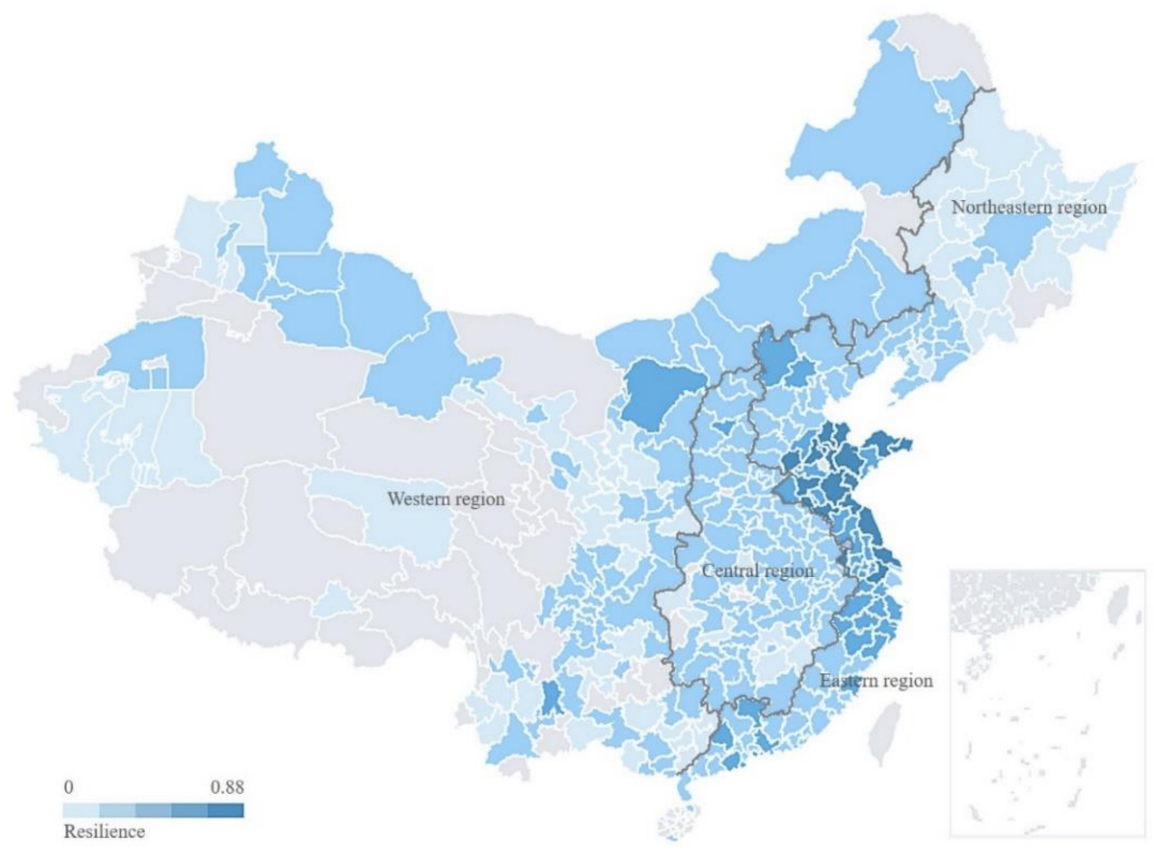

Figure 4. Resilience of urban energy systems for 309 Chinese cities. (Note: The gray areas were not included in the assessment because of lack of data.). 


\subsection{Regional Level}

In general, a majority of the 309 cities, especially those in the northeastern and western regions, had relatively low energy resilience. In contrast, UESR in the eastern region was generally higher. The average resilience $(R)$ result of the eastern region was more than twice that of the northeastern and western regions. The resilience variance $\left(S^{2}\right)$ of the eastern region was nearly an order of magnitude higher than that of the other three regions. The most evenly distributed cities were located in the central region. The differences in CV among the four regions were not significant in terms of average, maximum, minimum, or variance, with the eastern region only slightly higher than the other three regions. From the perspective of $\mathrm{CE}$, there were no obvious distribution characteristics. The eastern region had the highest average. The central region had the lowest variance. The situations of the western and northeastern regions were similar. The highest CI average occurred in the eastern region as well. The statistics of the evaluation results are shown in Table 1 . The detailed data and evaluation results can be seen in Tables S1-S4 of the Supplementary Materials.

Table 1. Statistics of the evaluation results.

\begin{tabular}{cccccc}
\hline Region & Resilience & $\mathbf{S}^{\mathbf{2}}$ & $\mathbf{C V}$ & $\mathbf{C E}$ & CI \\
\hline Nationwide & 0.32 & 0.022 & 0.36 & 0.20 & 0.36 \\
Western & 0.24 & 0.0053 & 0.35 & 0.16 & 0.34 \\
Central & 0.28 & 0.0028 & 0.35 & 0.18 & 0.36 \\
Eastern & 0.50 & 0.022 & 0.38 & 0.28 & 0.40 \\
Northeastern & 0.22 & 0.0035 & 0.37 & 0.16 & 0.33 \\
\hline
\end{tabular}

\subsection{Provincial Level}

Among the evaluated 27 provinces/autonomous regions:

- The highest average resilience occurred in Shandong (0.69), and the lowest, in Tibet (0.039). The distribution of resilience development was most balanced in Qinghai, with the lowest variance (0.000050) and the smallest range (0.020), and least balanced in Yunnan, with the second-highest variance (0.0046) and the largest range (0.26).

- The highest average CV occurred in Shandong (0.40), and the lowest, in Guizhou (0.32). The distribution of CV was most balanced in Tibet, with the lowest variance (0.000098) and the smallest range (0.028), and least balanced in Guangdong, with the highest variance (0.0046) and the largest range $(0.24)$.

- The highest average CE occurred in Shandong (0.36), and the lowest, in Tibet (0.049). The distribution of CE was most balanced in Qinghai, with the lowest variance (0.000057) and the smallest range (0.018), and least balanced in Ningxia, with the highest variance (0.0019) and the second-largest range (0.12).

- The highest average CI occurred in Jiangsu (0.41), and the lowest, in Tibet (0.26). The distribution of CI was most balanced in Hainan, with the lowest variance (0.000045) and the smallest range (0.016), and least balanced in Guangdong, with the highest variance $(0.0038)$ and the largest range $(0.25)$.

\subsection{City Level}

- Among the 309 cities, 107 (35\%) had higher energy resilience than the national average, while 202 (65\%) had lower energy resilience than the national average.

- The four municipalities, Tianjin, Shanghai, Chongqing, and Beijing, ranked 88th, 84th, 71 st, and 48th in resilience, respectively. All municipalities were above the average level, not only for resilience but for CV, CE and CI. Beijing ranked first in CI and CV.

- The minimum, median, and maximum resilience results corresponded to Rikaze, Yingkou, and Weifang, respectively. Detailed comparisons of these three cities are shown in Figures 5 and 6. The numbered acronyms on the left in Figure 6 correspond to the indices in Figure 3. The levels of the three cities' CV varied little. Rikaze had an obvious advantage in energy vulnerability, but its city vulnerability was due mainly to a large number of civil protection units in the city, such as historic sites, 
temples, and repositories of ancient books, pictographs, and other cultural relics. Its city competitiveness (index Fl 13-20), including the city's external connectivity, software and hardware environment, knowledge and information development level, and infrastructure construction, was in a disadvantageous position as well. These data were obtained from the Yearbook of China's Cities sponsored by the Sustainable City Committee of the China Research Society of Urban Development. According to the editor, the evaluation indices mainly reflected the competitiveness of cities in transforming from quantitative growth to qualitative sustainable development. To improve the resilience of Rikaze, this sustainable competitiveness should be comprehensively considered. Additionally, the reliability of the power supply can be improved, and the line loss rate of power enterprises can be reduced. Electricity conservation could be further advocated and executed, and new energy vehicles and enhanced transportation accessibility could be promoted. In terms of energy diversity, the use of natural gas and heat supply also lagged. However, this is related to the local climate and residents' habits and customs, which are difficult to change in the short term and require long-term adjustment and planning.

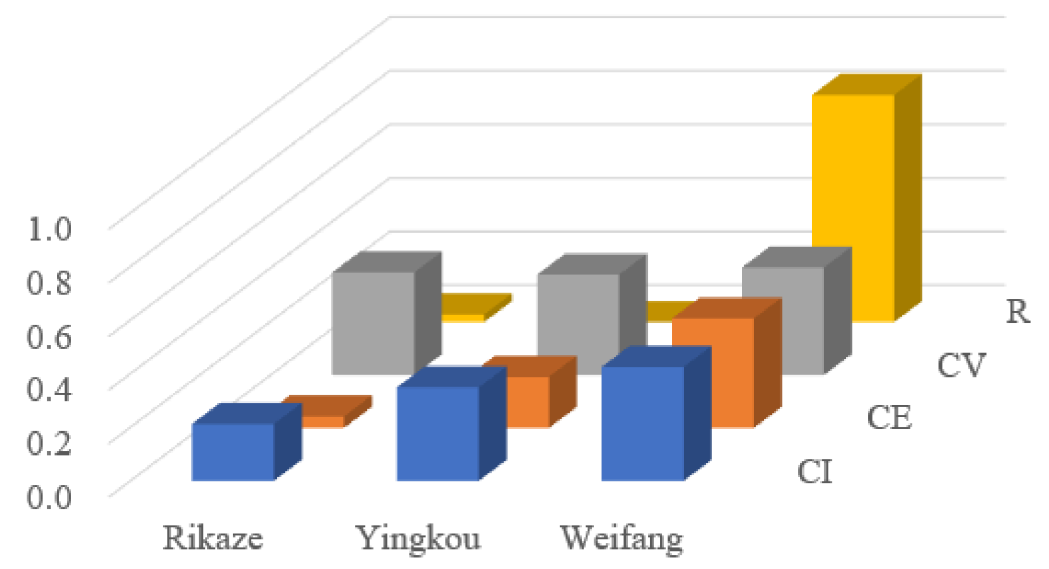

Figure 5. Comparison of the three cities' $\mathrm{R} / \mathrm{CV} / \mathrm{CE} / \mathrm{CI}$ results.

- $\quad$ For Yingkou, the main means of improving resilience would include promoting and practicing electricity conservation; improving the management of State Grid Liaoning Power Co., Ltd., among the major power grid companies in the country; and improving the diversity of power generation. With the current Huaneng Yingkou Thermal Power plant as the dominant plant, the city could develop microgrid projects, distributed energy systems, etc., to develop capacity other than thermal power generation.

- As the comparison of financial feasibility was based on provincial data, Weifang's advantages in both the fixed assets and current assets of the energy industry benefit from Shandong's advantages among provinces, as do the decommissioning of thermal power units and the achievement of energy savings. In addition, according to the China Electric Power Industry Annual Development Report, State Grid Shandong Power Co., Ltd., has relatively better comprehensive management on the supply side in its industry, so cities in Shandong also scored high on this series of indices. This implies that financial and managerial resilience can be improved at the provincial level.

\subsection{Regression Analysis}

Since the resilience of UESs is a critical issue in the current energy transition toward the 3060 targets, it is interesting to understand the relation among a city's energy system resilience, carbon dioxide emissions (megaton) and GDP $\left(10^{10} \mathrm{RMB}\right)$.

By the weighted least squares method (weight $=1 / \operatorname{resid}^{2}$ ), the following binary nonlinear regression equation is obtained, and the model fits the evaluation results well. 

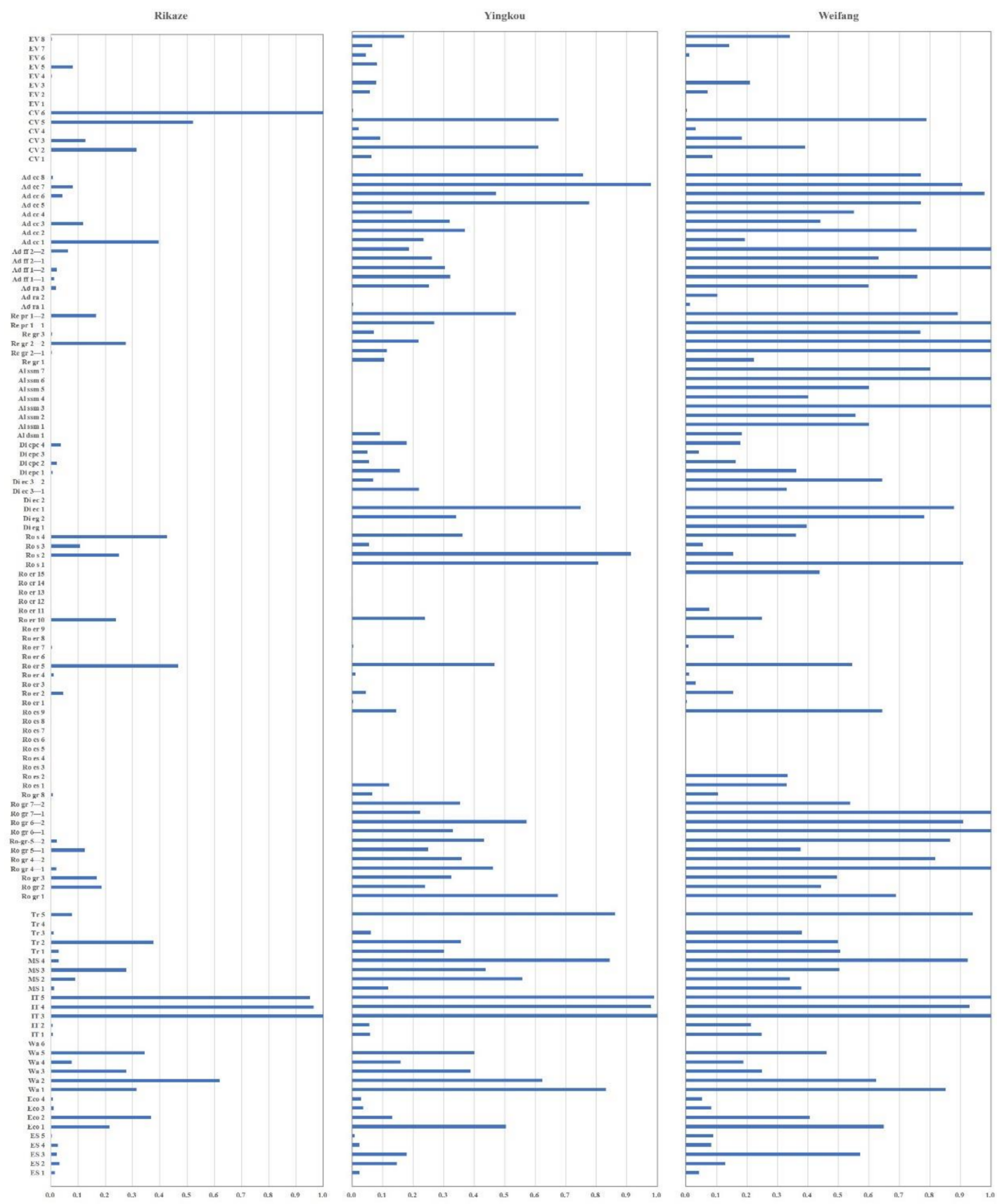

Figure 6. Comparison of cities with minimum/median/maximum resilience results.

$$
\begin{gathered}
\text { RESILIENCE }_{\mathrm{i}}=-0.049111+0.177735 \text { CO2 }_{\mathrm{i}}{ }^{0.204}+0.045861 \operatorname{lnGDP} \mathrm{i}_{\mathrm{i}}+\mathrm{e}_{\mathrm{i}} \\
\mathrm{t}=\left(705.8698^{* * *}\right)\left(749.1603^{* * *}\right)\left(484.5519^{* * *}\right) \\
\text { R_squared }=0.9999, \mathrm{n}=309
\end{gathered}
$$

where ${ }^{* * *}$ means at $1 \%$ significant level. The empirical results showed a positive correlation between resilience and carbon dioxide emissions, suggesting that there should be a balance among loss of resilience, reduction in carbon dioxide emissions, and increase in GDP. For an example, in Yingkou, a reduction in carbon dioxide emissions of one million tons would sacrifice resilience by 0.0073 and drop the city 12 places in the ranking, and an increase in GDP of 22,949.87 million RMB would enhance resilience to maintain the original position. Therefore, in the process of achieving the 3060 targets, to ensure the safety and sustainability of a city and allow its resilience to fluctuate within reasonable limits, how to appropriately 
allocate the carbon dioxide emission reduction quota to each city is critical. Based on the evaluation framework of this study, the options for both reducing emissions and enhancing resilience vary from city to city. Generally, feasible alternatives include advancing the financial feasibility of the energy sector, promoting, and practicing energy conservation, and improving the management of power enterprises.

\section{Conclusions}

With the ambitious 3060 targets, China is looking forward to an unprecedented energy transition. As a core part of energy transition and sustainability, resilience must be given serious attention, especially when extreme events have occurred more frequently in recent years.

To this end, this paper implemented a nationwide comprehensive assessment of the resilience of UESs in China. The results showed that the current capabilities of Chinese UESs to handle exogenous extreme events are very uneven, and that cities in the eastern region generally have higher resilience than those in other regions. The minimum, median, and maximum UESR results corresponded to Rikaze, Yingkou, and Weifang, respectively. Regression analysis of 309 cities' resilience evaluation results showed a positive correlation among UESR, carbon dioxide emissions, and GDP. When the details of this evaluation are combined and the differences lucubrated at the urban/provincial levels, each city should develop a tailored plan to reduce carbon emissions, ensure reasonable changes in UESR, and flexibly utilize economic instruments.

The aim of this study was to establish a benchmark to understand the complicated correlations and challenges of energy transition. The findings of this study may assist municipal and provincial decision makers with unique insights for enhancing overall UESR. Moreover, continual assessments of the UESR of these cities in future years could offer policy makers much more valuable information on energy transition and urban development.

The proposed indicators mainly suit China's current reality, and different, specific indices should be adopted when the assessments are applied to cities in other countries. The results do not contain value or other judgments.

Supplementary Materials: The following are available online at https:/ /www.mdpi.com/article/10 .3390 / su14042077/s1, Table S1: Resilience evaluation results of 309 Chinese cities, Table S2: CI data and results of 309 Chinese cities, Table S3: CE data and results of 309 Chinese cities, Table S4: CV data and results of 309 Chinese cities.

Author Contributions: Conceptualization, Z.W. and R.W.; methodology, Z.W. and Q.S.; software, Z.W.; validation, Z.W., C.M. and Q.S.; investigation, Z.W. and Z.C.; resources, Q.S.; data curation, Z.W. and Z.C.; writing—original draft preparation, Z.W.; writing—review and editing, Z.W.; supervision, R.W. All authors have read and agreed to the published version of the manuscript.

Funding: This research received no external funding.

Institutional Review Board Statement: Not applicable.

Informed Consent Statement: Not applicable.

Data Availability Statement: Data sources included scholarly publications, trade organization publications, research reports produced by governmental departments and educational organizations, and, when possible, direct contact with experts in related fields. In detail, the CI data sources included governmental yearbooks and bulletins at the city/provincial/country levels, the academic research results of transportation accessibility in [40], and the China Urban Construction Statistical Yearbook. The CE data sources included governmental yearbooks and bulletins at the city/provincial/country levels; the business inquiry platform www.tianyancha.com (accessed on 22 May 2021); the official website of the Ministry of Industry and Information Technology of the People's Republic of China, https:/ / www.miit.gov.cn/ (accessed on 1 February 2022); the official website of the National Development and Reform Commission of the People's Republic of China, https:/ / www.ndrc.gov.cn/ (accessed on 1 February 2022); and the China Urban Construction Statistical Yearbook, China Electric Power Yearbook, China Electric Power Statistical Yearbook, State Grid Yearbook, China Electric 
Power Industry Annual Development Report, China Automobile Industry Yearbook, China Industrial Statistical Yearbook, Yearbook of China's Cities, and China Basic Unit Statistical Yearbook. The CV data sources included the China Urban Construction Statistical Yearbook and the China Economic and Social Big Data Research Platform, https://data.cnki.net/NewHome/index (accessed on 1 February 2022).

Acknowledgments: This work was supported by the Shandong University Seed Fund Program for International Research Cooperation.

Conflicts of Interest: The authors declare no conflict of interest.

\section{Appendix A}

Table A1. Aggregated index selection for CE (note: $\checkmark$ indicates compliance with the selection principle and $X$ indicates noncompliance; selection principles: systematism (S), unicity (U), feasibility $(\mathrm{F})$, objectivity $(\mathrm{O})$, and representation $(\mathrm{R})$ ).

\begin{tabular}{|c|c|c|c|c|c|c|c|c|}
\hline No. & Primary Index & Ref. & S & $\mathbf{U}$ & $\mathbf{F}$ & $\mathbf{O}$ & $\mathbf{R}$ & Result \\
\hline 1 & Energy feedstock & [52] & $\checkmark$ & $\checkmark$ & $x$ & $\checkmark$ & $\checkmark$ & Deleted \\
\hline 2 & Energy not supplied & [53] & $\checkmark$ & $\checkmark$ & $x$ & $x$ & $\checkmark$ & Deleted \\
\hline 3 & Energy storage & [54] & $\checkmark$ & $\checkmark$ & $\checkmark$ & $\checkmark$ & $\checkmark$ & Retained \\
\hline 4 & Hydrophobic coating on equipment & [55] & $x$ & $\checkmark$ & $x$ & $\checkmark$ & $x$ & Deleted \\
\hline 5 & Key replacement equipment stockpile & [55] & $x$ & $\checkmark$ & $x$ & $\checkmark$ & $x$ & Deleted \\
\hline 6 & Redundant power lines & [55] & $\checkmark$ & $\checkmark$ & $x$ & $\checkmark$ & $x$ & Deleted \\
\hline 7 & $\begin{array}{l}\text { Reinforced concrete versus wooden } \\
\text { distribution poles }\end{array}$ & [55] & $x$ & $\checkmark$ & $x$ & $x$ & $\checkmark$ & Deleted \\
\hline 8 & Siting infrastructure & [55] & $x$ & $\checkmark$ & $x$ & $\checkmark$ & $x$ & Deleted \\
\hline 9 & $\begin{array}{l}\text { Underground, overhead, undersea } \\
\text { distribution/cable lines }\end{array}$ & {$[56,57]$} & $\checkmark$ & $\checkmark$ & $x$ & $\checkmark$ & $\checkmark$ & Deleted \\
\hline 10 & $\begin{array}{l}\text { Unique encrypted passwords for utility } \\
\text { "smart" distribution }\end{array}$ & [55] & $x$ & $\checkmark$ & $x$ & $x$ & $x$ & Deleted \\
\hline 11 & Workers employed & {$[52,55,58]$} & $\checkmark$ & $\checkmark$ & $\checkmark$ & $\checkmark$ & $\checkmark$ & Retained \\
\hline 12 & $\begin{array}{l}\text { Communication/control } \\
\text { systems/control centers }\end{array}$ & [59] & $x$ & $\checkmark$ & $x$ & $\checkmark$ & $\checkmark$ & Deleted \\
\hline 13 & Electrical protection and metering & [59] & $x$ & $\checkmark$ & $x$ & $\checkmark$ & $x$ & Deleted \\
\hline 14 & Equipment positioning & [55] & $x$ & $\checkmark$ & $x$ & $x$ & $x$ & Deleted \\
\hline 15 & Flow paths, line flow limits & {$[60]$} & $x$ & $\checkmark$ & $x$ & $\checkmark$ & $x$ & Deleted \\
\hline 16 & Gen/load bus distribution & [60] & $x$ & $\checkmark$ & $x$ & $\checkmark$ & $x$ & Deleted \\
\hline 17 & Reserve/spare capacity & {$[57,61,62]$} & $\checkmark$ & $\checkmark$ & $\checkmark$ & $\checkmark$ & $\checkmark$ & Retained \\
\hline 18 & $\begin{array}{l}\text { Substations (switchyards)_overhead } \\
\text { lines and underground cables } \\
\text { are interconnected }\end{array}$ & [59] & $x$ & $\checkmark$ & $x$ & $\checkmark$ & $x$ & Deleted \\
\hline 19 & Ancillary service & [54] & $x$ & $\checkmark$ & $x$ & $x$ & $\checkmark$ & Deleted \\
\hline 20 & $\begin{array}{c}\text { Function-altered hazard rate of } \\
\text { component after certain maintenance }\end{array}$ & [63] & $x$ & $\checkmark$ & $x$ & $\checkmark$ & $\checkmark$ & Deleted \\
\hline 21 & $\begin{array}{l}\text { Net ability-measures the aptitude of } \\
\text { the grid in transmitting power from } \\
\text { generation to load buses efficiently }\end{array}$ & [60] & $\checkmark$ & $\checkmark$ & $x$ & $\checkmark$ & $\checkmark$ & Deleted \\
\hline 22 & $\begin{array}{l}\text { Path redundancy-assesses the } \\
\text { available redundancy in terms of paths } \\
\text { in transmitting power from generation } \\
\text { to a load bus based on entropy }\end{array}$ & [60] & $\checkmark$ & $\checkmark$ & $x$ & $x$ & $\checkmark$ & Deleted \\
\hline 23 & Viability of investments & [52] & $x$ & $\checkmark$ & $x$ & $x$ & $\checkmark$ & Deleted \\
\hline 24 & $\begin{array}{l}\text { Coefficient of variation of the frequency } \\
\text { index of sags }\end{array}$ & [64] & $x$ & $\checkmark$ & $x$ & $\checkmark$ & $\checkmark$ & Deleted \\
\hline 25 & $\begin{array}{l}\text { Bulk electric system reliability } \\
\text { performance indices }\end{array}$ & [65] & $\checkmark$ & $\checkmark$ & $\checkmark$ & $\checkmark$ & $\checkmark$ & Retained \\
\hline 26 & $\begin{array}{l}\text { Derated power-rated power } \\
\text { multiplied by the reliability of the plant }\end{array}$ & [66] & $x$ & $\checkmark$ & $x$ & $\checkmark$ & $\checkmark$ & Deleted \\
\hline
\end{tabular}


Table A1. Cont.

\begin{tabular}{|c|c|c|c|c|c|c|c|c|}
\hline No. & Primary Index & Ref. & S & U & $\mathbf{F}$ & $\mathbf{O}$ & $\mathbf{R}$ & Result \\
\hline 27 & Energy efficiency/intensity & {$[62,67-70]$} & $\checkmark$ & $\checkmark$ & $\checkmark$ & $\checkmark$ & $\checkmark$ & Retained \\
\hline 28 & Failure rate & [63] & $x$ & $\checkmark$ & $\checkmark$ & $\checkmark$ & $x$ & Deleted \\
\hline 29 & $\begin{array}{l}\text { Resilience index - parameter that } \\
\text { quantifies the potential probability of } \\
\text { malfunction of the system }\end{array}$ & [71] & $\checkmark$ & $\checkmark$ & $\checkmark$ & $\checkmark$ & $\checkmark$ & Retained \\
\hline 30 & $\begin{array}{l}\text { Resilience index_derived from } \\
\text { robustness, resourcefulness, and } \\
\text { recovery; ranges from } 0 \text { (low resilience) } \\
\text { to } 100 \text { (high resilience) }\end{array}$ & {$[30,72,73]$} & $\checkmark$ & $\checkmark$ & $\checkmark$ & $\checkmark$ & $\checkmark$ & Retained \\
\hline 31 & $\begin{array}{l}\text { Survivability - evaluates the aptitude } \\
\text { of the network to assure the possibility } \\
\text { of matching generation and demand in } \\
\text { case of failures or attacks }\end{array}$ & [60] & $\checkmark$ & $\checkmark$ & $x$ & $x$ & $\checkmark$ & Deleted \\
\hline 32 & $\begin{array}{l}\text { System average interruption } \\
\text { duration/frequency index }\end{array}$ & [74] & $x$ & $\checkmark$ & $x$ & $\checkmark$ & $\checkmark$ & Deleted \\
\hline 33 & $\begin{array}{l}\text { Load loss damage index-damage } \\
\text { caused by fire to the electrical system }\end{array}$ & [75] & $x$ & $\checkmark$ & $x$ & $\checkmark$ & $x$ & Deleted \\
\hline 34 & Transmission lines available & [76] & $\checkmark$ & $\checkmark$ & $\checkmark$ & $\checkmark$ & $\checkmark$ & Retained \\
\hline 35 & $\begin{array}{l}\text { Functional zones-generation, } \\
\text { transmission, and distribution }\end{array}$ & [52] & $\checkmark$ & $\checkmark$ & $\checkmark$ & $\checkmark$ & $\checkmark$ & Retained \\
\hline 36 & Operator training & [55] & $x$ & $\checkmark$ & $x$ & $x$ & $\checkmark$ & Deleted \\
\hline 37 & Mutual assistant agreements & [55] & $\checkmark$ & $\checkmark$ & $x$ & $x$ & $\checkmark$ & Deleted \\
\hline 38 & $\begin{array}{l}\text { Transformers-connecting parts of the } \\
\text { network operating at different voltages }\end{array}$ & [59] & $\checkmark$ & $\checkmark$ & $\checkmark$ & $\checkmark$ & $\checkmark$ & Retained \\
\hline 39 & Tree-trimming metrics & {$[55,57]$} & $\checkmark$ & $\checkmark$ & $x$ & $x$ & $\checkmark$ & Deleted \\
\hline 40 & $\begin{array}{l}\text { Adequacy-the ability of the system to } \\
\text { supply customer requirements under } \\
\text { normal operating conditions }\end{array}$ & [52] & $\checkmark$ & $\checkmark$ & $\checkmark$ & $\checkmark$ & $x$ & Deleted \\
\hline 41 & Congestion control & [77] & $x$ & $\checkmark$ & $x$ & $\checkmark$ & $\checkmark$ & Deleted \\
\hline 42 & $\begin{array}{l}\text { Customer average interruption } \\
\text { duration index-sustained outage } \\
\text { metric; measures average duration of } \\
\text { sustained outage per customer }\end{array}$ & [74] & $x$ & $\checkmark$ & $x$ & $\checkmark$ & $\checkmark$ & Deleted \\
\hline 43 & $\begin{array}{l}\text { Economy-achieving the best profits } \\
\text { by adjusting the power system } \\
\text { operation mode to minimize line losses, } \\
\text { making full use of equipment, ensuring } \\
\text { the security of the power system, and } \\
\text { meeting utility users' demand }\end{array}$ & [68] & $\checkmark$ & $\checkmark$ & $x$ & $x$ & $\checkmark$ & Deleted \\
\hline 44 & $\begin{array}{l}\text { Fairness - consists of the fulfillment } \\
\text { rate of contracts and standard } \\
\text { deviation indexes }\end{array}$ & [68] & $x$ & $\checkmark$ & $x$ & $x$ & $x$ & Deleted \\
\hline 45 & Interrupted energy assessment rate & [65] & $x$ & $\checkmark$ & $x$ & $\checkmark$ & $\checkmark$ & Deleted \\
\hline 46 & $\begin{array}{l}\text { Security-the dynamic response of the } \\
\text { system to unexpected interruptions; } \\
\text { relates to the system's ability to } \\
\text { endure them }\end{array}$ & [52] & $\checkmark$ & $\checkmark$ & $x$ & $x$ & $\checkmark$ & Deleted \\
\hline 47 & Transmission losses & [56] & $\checkmark$ & $\checkmark$ & $\checkmark$ & $\checkmark$ & $\checkmark$ & Retained \\
\hline 48 & $\begin{array}{l}\text { Cost of interruption-social, } \\
\text { commercial, industrial, etc. }\end{array}$ & {$[56]$} & $\checkmark$ & $\checkmark$ & $x$ & $x$ & $\checkmark$ & Deleted \\
\hline 49 & $\begin{array}{c}\text { Impact factor on the population-share } \\
\text { of the population affected by the } \\
\text { power loss }\end{array}$ & [78] & $\checkmark$ & $x$ & $x$ & $\checkmark$ & $\checkmark$ & Deleted \\
\hline 50 & Long-distance transmission costs & [56] & $\checkmark$ & $x$ & $\checkmark$ & $\checkmark$ & $\checkmark$ & Deleted \\
\hline 51 & Noise & [56] & $x$ & $\checkmark$ & $x$ & $x$ & $\checkmark$ & Deleted \\
\hline 52 & $\begin{array}{l}\text { Performance-based regulation } \\
\text { reward/penalty structure }\end{array}$ & {$[65]$} & $x$ & $\checkmark$ & $x$ & $x$ & $\checkmark$ & Deleted \\
\hline 53 & Price of electricity & [56] & $\checkmark$ & $\checkmark$ & $\checkmark$ & $\checkmark$ & $\checkmark$ & Retained \\
\hline
\end{tabular}


Table A1. Cont.

\begin{tabular}{|c|c|c|c|c|c|c|c|c|}
\hline No. & Primary Index & Ref. & $\mathrm{S}$ & $\mathrm{U}$ & $\mathbf{F}$ & O & $\mathbf{R}$ & Result \\
\hline 54 & $\begin{array}{l}\text { Value of lost load-value of unserved } \\
\text { energy; customers' value of the } \\
\text { opportunity cost of outages or benefits } \\
\text { forgone through interruptions in } \\
\text { electricity supply }\end{array}$ & [61] & $\checkmark$ & $\checkmark$ & $x$ & $x$ & $\checkmark$ & Deleted \\
\hline 55 & $\begin{array}{l}\text { Fuel nodes with the most links are the } \\
\text { most interconnected and serve as hubs } \\
\text { Flow between nodes takes place on }\end{array}$ & [79] & $\checkmark$ & $\checkmark$ & $x$ & $\checkmark$ & $x$ & Deleted \\
\hline 56 & $\begin{array}{l}\text { links (roads, electric power } \\
\text { transmission lines, water mains, etc.) }\end{array}$ & [79-81] & $x$ & $\checkmark$ & $x$ & $x$ & $\checkmark$ & Deleted \\
\hline 57 & $\begin{array}{l}\text { Elements of the energy network that } \\
\text { can receive fuels from storage facilities, } \\
\text { pipeline interconnections, or } \\
\text { production areas }\end{array}$ & {$[79,81]$} & $\checkmark$ & $\checkmark$ & $\checkmark$ & $\checkmark$ & $\checkmark$ & Retained \\
\hline 58 & $\begin{array}{l}\text { Primary energy supply-includes the } \\
\text { systems and processes used to supply a } \\
\text { primary energy resource to its point of } \\
\text { conversion into the final energy } \\
\text { product of interest }\end{array}$ & [52] & $\checkmark$ & $\checkmark$ & $\checkmark$ & $\checkmark$ & $\checkmark$ & Retained \\
\hline 59 & $\begin{array}{l}\text { Storage facilities/nodes, } \\
\text { intermediate storage }\end{array}$ & {$[80,81]$} & $\checkmark$ & $\checkmark$ & $\checkmark$ & $\checkmark$ & $\checkmark$ & Retained \\
\hline 60 & $\begin{array}{l}\text { Emergency procedures/emergency } \\
\text { shutdown system }\end{array}$ & [82] & $\checkmark$ & $\checkmark$ & $x$ & $x$ & $\checkmark$ & Deleted \\
\hline 61 & $\begin{array}{l}\text { Response to equipment } \\
\text { outages-degree to which the system is } \\
\text { able to continue to reliably operate in } \\
\text { the event of equipment downtime }\end{array}$ & [52] & $x$ & $\checkmark$ & $x$ & $x$ & $\checkmark$ & Deleted \\
\hline 62 & $\begin{array}{l}\text { Adaptive capacity - degree to which } \\
\text { the system is capable of } \\
\text { self-organization for recovery of system } \\
\text { performance levels }\end{array}$ & [83] & $\checkmark$ & $\checkmark$ & $x$ & $x$ & $\checkmark$ & Deleted \\
\hline 63 & $\begin{array}{l}\text { Ability of the system to provide } \\
\text { sufficient throughput to supply } \\
\text { final demand }\end{array}$ & [52] & $\checkmark$ & $\checkmark$ & $\checkmark$ & $\checkmark$ & $\checkmark$ & Retained \\
\hline 64 & $\begin{array}{l}\text { Information security-the degree to } \\
\text { which information assets in the system } \\
\text { are secure against threats }\end{array}$ & [52] & $\checkmark$ & $\checkmark$ & $\checkmark$ & $\checkmark$ & $\checkmark$ & Retained \\
\hline 65 & $\begin{array}{l}\text { Physical security-the degree towhich } \\
\text { physical assets in the systemare secure } \\
\text { against threats }\end{array}$ & [52] & $\checkmark$ & $\checkmark$ & $x$ & $x$ & $\checkmark$ & Deleted \\
\hline 66 & $\begin{array}{l}\text { Absorptive capacity-degree to which } \\
\text { a system can automatically absorb the } \\
\text { impacts of perturbations and minimize } \\
\text { consequences with little effort }\end{array}$ & [83] & $\checkmark$ & $\checkmark$ & $x$ & $x$ & $\checkmark$ & Deleted \\
\hline 67 & $\begin{array}{l}\text { Connectivity loss - the average } \\
\text { reduction in the ability of sinks to } \\
\text { receive flow from sources }\end{array}$ & [78] & $\checkmark$ & $x$ & $\checkmark$ & $\checkmark$ & $\checkmark$ & Deleted \\
\hline 68 & $\begin{array}{l}\text { Energy processing and } \\
\text { conversion-relates to production of } \\
\text { the final energy product }\end{array}$ & [52] & $\checkmark$ & $\checkmark$ & $\checkmark$ & $\checkmark$ & $\checkmark$ & Retained \\
\hline 69 & $\begin{array}{l}\text { Flexibility - the degree to which the } \\
\text { system can adapt to changing } \\
\text { conditions }\end{array}$ & [52] & $\checkmark$ & $\checkmark$ & $x$ & $x$ & $\checkmark$ & Deleted \\
\hline 70 & $\begin{array}{c}\text { History-the degree to which the } \\
\text { system has been prone to disruption in } \\
\text { the past }\end{array}$ & [52] & $\checkmark$ & $\checkmark$ & $\checkmark$ & $\checkmark$ & $\checkmark$ & Retained \\
\hline
\end{tabular}


Table A1. Cont.

\begin{tabular}{|c|c|c|c|c|c|c|c|c|}
\hline No. & Primary Index & Ref. & $\mathrm{S}$ & U & $\mathbf{F}$ & $\mathbf{O}$ & $\mathbf{R}$ & Result \\
\hline 71 & $\begin{array}{c}\text { Intermittency - the degree to which the } \\
\text { system lacks constant levels } \\
\text { of productivity }\end{array}$ & [52] & $x$ & $\checkmark$ & $x$ & $x$ & $x$ & Deleted \\
\hline 72 & $\begin{array}{l}\text { Network resiliency-measured by its } \\
\text { ability to keep supplying and distributing } \\
\text { fuels in spite of damage to pipelines, } \\
\text { import terminals, storage, and } \\
\text { other sources }\end{array}$ & [79] & $\checkmark$ & $\checkmark$ & $x$ & $x$ & $\checkmark$ & Deleted \\
\hline 73 & $\begin{array}{l}\text { Response to demand fluctuations-the } \\
\text { extent to which the system is able to adapt } \\
\text { to changes in the quantity of energy } \\
\text { demanded or location of demand }\end{array}$ & [52] & $\checkmark$ & $\checkmark$ & $\checkmark$ & $\checkmark$ & $\checkmark$ & Retained \\
\hline 74 & $\begin{array}{l}\text { Systemic impact-impact that a } \\
\text { disruption has on system productivity; } \\
\text { measured by evaluating the difference } \\
\text { between a targeted system performance } \\
\text { level and the actual system performance }\end{array}$ & {$[80,83]$} & $\checkmark$ & $\checkmark$ & $x$ & $x$ & $x$ & Deleted \\
\hline 75 & $\begin{array}{l}\text { Impacts on interdependent systems-the } \\
\text { degree to which a disruption in the } \\
\text { system might feasibly cause damage to } \\
\text { interdependent systems }\end{array}$ & [52] & $\checkmark$ & $\checkmark$ & $x$ & $x$ & $\checkmark$ & Deleted \\
\hline 76 & $\begin{array}{l}\text { Optimal resilience costs-resilience costs } \\
\text { for a system when the optimal recovery } \\
\text { strategy (minimizing the combined } \\
\text { system impact and total recovery effort } \\
\text { costs) is employed }\end{array}$ & [83] & $x$ & $\checkmark$ & $x$ & $x$ & $\checkmark$ & Deleted \\
\hline 77 & $\begin{array}{l}\text { Recovery-dependent resilience } \\
\text { costs—resilience costs of a system under a } \\
\text { particular recovery strategy }\end{array}$ & [83] & $x$ & $\checkmark$ & $x$ & $x$ & $\checkmark$ & Deleted \\
\hline 78 & Diversity of import fuels & [67] & $x$ & $\checkmark$ & $x$ & $\checkmark$ & $\checkmark$ & Deleted \\
\hline 79 & Natural gas strategic reserve & [84] & $\checkmark$ & $\checkmark$ & $\checkmark$ & $\checkmark$ & $\checkmark$ & Retained \\
\hline 80 & $\begin{array}{l}\text { Import levels-the degree to which } \\
\text { primary energy supply relies on resources } \\
\text { originating outside of the system }\end{array}$ & $\begin{array}{l}{[17,52,62} \\
81,85-92]\end{array}$ & $\checkmark$ & $\checkmark$ & $\checkmark$ & $\checkmark$ & $\checkmark$ & Retained \\
\hline 81 & $\begin{array}{l}\text { Industrial aspects—vulnerability indicator } \\
\text { Vulnerability - proportional to the }\end{array}$ & [85] & $\checkmark$ & $\checkmark$ & $x$ & $x$ & $\checkmark$ & Deleted \\
\hline 82 & $\begin{array}{l}\text { reliance on imported gas from countries in } \\
\text { geopolitical conflict }\end{array}$ & [85] & $x$ & $\checkmark$ & $x$ & $\checkmark$ & $\checkmark$ & Deleted \\
\hline 83 & $\begin{array}{l}\text { Ability to expand facilities-the degree to } \\
\text { which the system can be easily and } \\
\text { cost-effectively expanded }\end{array}$ & [52] & $\checkmark$ & $\checkmark$ & $x$ & $x$ & $\checkmark$ & Deleted \\
\hline 84 & Pipeline capacity used & [79] & $\checkmark$ & $\checkmark$ & $x$ & $\checkmark$ & $\checkmark$ & Deleted \\
\hline 85 & $\begin{array}{l}\text { Resiliency_ability to supply gas to } \\
\text { customers willing to pay the clearing } \\
\text { price, even in the face of } \\
\text { supply constraints }\end{array}$ & [84] & $x$ & $\checkmark$ & $x$ & $x$ & $\checkmark$ & Deleted \\
\hline 86 & $\begin{array}{l}\text { Restorative capacity - ability of a system } \\
\text { to be repaired easily; these repairs are } \\
\text { considered to be dynamic }\end{array}$ & [83] & $\checkmark$ & $\checkmark$ & $x$ & $x$ & $\checkmark$ & Deleted \\
\hline 87 & $\begin{array}{l}\text { Total recovery effort- efficiency with } \\
\text { which a system recovers from a } \\
\text { disruption, measured by analyzing the } \\
\text { amount of resources expended during the } \\
\text { recovery process }\end{array}$ & [83] & $\checkmark$ & $\checkmark$ & $x$ & $x$ & $\checkmark$ & Deleted \\
\hline 88 & $\begin{array}{l}\text { Sector coordination-the degree to which } \\
\text { coordination between stakeholders within } \\
\text { the sector results in an effective exchange } \\
\text { of information, alerting stakeholders of } \\
\text { emerging threats and mitigation strategies }\end{array}$ & [52] & $\checkmark$ & $\checkmark$ & $\checkmark$ & $\checkmark$ & $\checkmark$ & Retained \\
\hline
\end{tabular}


Table A1. Cont.

\begin{tabular}{|c|c|c|c|c|c|c|c|c|}
\hline No. & Primary Index & Ref. & $\mathbf{S}$ & $\mathbf{U}$ & $\mathbf{F}$ & $\mathbf{O}$ & $\mathbf{R}$ & Result \\
\hline 89 & Price/price volatility & {$[52,84]$} & $\checkmark$ & $\checkmark$ & $\checkmark$ & $\checkmark$ & $\checkmark$ & Retained \\
\hline 90 & $\begin{array}{l}\text { Intelligent institutional leadership with } \\
\text { heightened sensitivity and/or preparedness } \\
\text { for rapid and pervasive changes }\end{array}$ & [93] & $x$ & $\checkmark$ & $x$ & $x$ & $\checkmark$ & Deleted \\
\hline 91 & Diversity of electricity generation & $\begin{array}{l}{[16,17,31,34,62,} \\
86-91,94-108]\end{array}$ & $\checkmark$ & $\checkmark$ & $\checkmark$ & $\checkmark$ & $\checkmark$ & Retained \\
\hline 92 & Diversity of imports of embodied electricity & [34] & $x$ & $\checkmark$ & $x$ & $\checkmark$ & $\checkmark$ & Deleted \\
\hline 93 & Diversity of electricity consumption & [34] & $\checkmark$ & $\checkmark$ & $\checkmark$ & $\checkmark$ & $\checkmark$ & Retained \\
\hline 94 & $\begin{array}{c}\text { Renewable energy electricity, mainly wind } \\
\text { and solar power }\end{array}$ & [109-111] & $\checkmark$ & $\checkmark$ & $\checkmark$ & $\checkmark$ & $\checkmark$ & Retained \\
\hline 95 & $\begin{array}{l}\text { Share of buildings with low thermal } \\
\text { insulation in the total building stock }\end{array}$ & [112] & $\checkmark$ & $\checkmark$ & $x$ & $x$ & $\checkmark$ & Deleted \\
\hline 96 & Share of renewables in total heating energy & [112] & $\checkmark$ & $\checkmark$ & $x$ & $\checkmark$ & $\checkmark$ & Deleted \\
\hline 97 & $\begin{array}{c}\text { Share of fossil fuels in total } \\
\text { energy consumption }\end{array}$ & [112] & $\checkmark$ & $x$ & $\checkmark$ & $\checkmark$ & $\checkmark$ & Deleted \\
\hline 98 & $\begin{array}{l}\text { Share of electricity produced by renewables } \\
\text { in total electricity consumption }\end{array}$ & {$[8,112]$} & $\checkmark$ & $x$ & $\checkmark$ & $\checkmark$ & $\checkmark$ & Deleted \\
\hline 99 & Nonrenewable fuel used in generation & [62] & $\checkmark$ & $x$ & $\checkmark$ & $\checkmark$ & $\checkmark$ & Deleted \\
\hline 100 & Generation efficiency & {$[62]$} & $\checkmark$ & $x$ & $\checkmark$ & $\checkmark$ & $\checkmark$ & Deleted \\
\hline 101 & $\begin{array}{l}\text { Distribution efficiency-transmission and } \\
\text { distribution losses and the amount of } \\
\text { electricity consumed by energy industry }\end{array}$ & [62] & $\checkmark$ & $x$ & $x$ & $\checkmark$ & $\checkmark$ & Deleted \\
\hline 102 & Carbon intensity of generation & $\begin{array}{l}{[17,49,62,87,91} \\
98,113]\end{array}$ & $\checkmark$ & $x$ & $x$ & $\checkmark$ & $x$ & Deleted \\
\hline 103 & Redundant power for use & [62] & $\checkmark$ & $\checkmark$ & $\checkmark$ & $\checkmark$ & $\checkmark$ & Retained \\
\hline 104 & $\begin{array}{l}\text { Existence and monitoring of officially } \\
\text { approved electrification plan }\end{array}$ & [114] & $x$ & $\checkmark$ & $x$ & $x$ & $x$ & Deleted \\
\hline 105 & Framework for grid electrification & [114] & $x$ & $\checkmark$ & $x$ & $x$ & $\checkmark$ & Deleted \\
\hline 106 & Framework for minigrids & [114] & $x$ & $\checkmark$ & $x$ & $x$ & $\checkmark$ & Deleted \\
\hline 107 & Framework for standalone systems & [114] & $\checkmark$ & $\checkmark$ & $x$ & $x$ & $\checkmark$ & Deleted \\
\hline 108 & Consumer affordability of electricity & {$[110,114]$} & $\checkmark$ & $\checkmark$ & $x$ & $x$ & $\checkmark$ & Deleted \\
\hline 109 & Utility transparency and monitoring & [114] & $x$ & $\checkmark$ & $x$ & $x$ & $x$ & Deleted \\
\hline 110 & Utility creditworthiness & [114] & $x$ & $\checkmark$ & $x$ & $x$ & $x$ & Deleted \\
\hline 111 & $\begin{array}{l}\text { Information provided to consumers about } \\
\text { electricity usage }\end{array}$ & [114] & $\checkmark$ & $\checkmark$ & $x$ & $x$ & $\checkmark$ & Deleted \\
\hline 112 & Financing mechanisms for energy efficiency & [114] & $\checkmark$ & $\checkmark$ & $x$ & $x$ & $\checkmark$ & Deleted \\
\hline 113 & Energy efficiency entities & [114] & $x$ & $\checkmark$ & $x$ & $x$ & $\checkmark$ & Deleted \\
\hline 114 & Incentives from electricity rate structures & [114] & $x$ & $\checkmark$ & $x$ & $x$ & $\checkmark$ & Deleted \\
\hline 115 & $\begin{array}{l}\text { Incentives and mandates: large } \\
\text { consumers/public sector/utilities }\end{array}$ & [114] & $x$ & $\checkmark$ & $x$ & $x$ & $\checkmark$ & Deleted \\
\hline 116 & $\begin{array}{l}\text { Minimum energy efficiency } \\
\text { performance standards }\end{array}$ & [114] & $\checkmark$ & $\checkmark$ & $x$ & $x$ & $\checkmark$ & Deleted \\
\hline 117 & Energy labeling systems & [114] & $\checkmark$ & $\checkmark$ & $x$ & $x$ & $\checkmark$ & Deleted \\
\hline 118 & Building energy codes & [114] & $\checkmark$ & $\checkmark$ & $x$ & $x$ & $\checkmark$ & Deleted \\
\hline 119 & Carbon pricing and monitoring & {$[95,114-117]$} & $\checkmark$ & $\checkmark$ & $x$ & $x$ & $\checkmark$ & Deleted \\
\hline 120 & Legal framework for renewable energy & [114] & $\checkmark$ & $\checkmark$ & $x$ & $x$ & $\checkmark$ & Deleted \\
\hline 121 & Planning for renewable energy expansion & [114] & $\checkmark$ & $x$ & $x$ & $x$ & $\checkmark$ & Deleted \\
\hline 122 & $\begin{array}{c}\text { Incentives and regulatory support for } \\
\text { renewable energy }\end{array}$ & [114] & $\checkmark$ & $\checkmark$ & $x$ & $x$ & $\checkmark$ & Deleted \\
\hline 123 & $\begin{array}{l}\text { Attributes of financial and regulatory } \\
\text { incentives for renewable energy }\end{array}$ & [114] & $\checkmark$ & $\checkmark$ & $x$ & $x$ & $\checkmark$ & Deleted \\
\hline 124 & Network connection and pricing & [114] & $\checkmark$ & $x$ & $x$ & $x$ & $\checkmark$ & Deleted \\
\hline 125 & Counterparty risk of renewable energy & [114] & $x$ & $\checkmark$ & $x$ & $x$ & $x$ & Deleted \\
\hline 126 & $\begin{array}{l}\text { Maximized availability of operational } \\
\text { power supply }\end{array}$ & [118] & $x$ & $\checkmark$ & $x$ & $x$ & $\checkmark$ & Deleted \\
\hline 127 & $\begin{array}{c}\text { Replacement inventories of equipment } \\
\text { and supplies }\end{array}$ & {$[110,118]$} & $\checkmark$ & $\checkmark$ & $x$ & $x$ & $\checkmark$ & Deleted \\
\hline
\end{tabular}


Table A1. Cont.

\begin{tabular}{|c|c|c|c|c|c|c|c|c|}
\hline No. & Primary Index & Ref. & $\mathbf{S}$ & $\mathbf{U}$ & $\mathbf{F}$ & $\mathbf{O}$ & $\mathbf{R}$ & Result \\
\hline 128 & $\begin{array}{c}\text { Maximized provision target power supply } \\
\text { level of restoration }\end{array}$ & [118] & $\checkmark$ & $\checkmark$ & $x$ & $x$ & $\checkmark$ & Deleted \\
\hline 129 & Largest single source of supply & [17] & $\checkmark$ & $\checkmark$ & $\checkmark$ & $\checkmark$ & $x$ & Deleted \\
\hline 130 & Energy portfolios—price volatility & [17] & $\checkmark$ & $x$ & $\checkmark$ & $\checkmark$ & $\checkmark$ & Deleted \\
\hline 131 & $\begin{array}{l}\text { Statistical probability of supply interruption } \\
\text { in network industries (gas and electricity) }\end{array}$ & [17] & $x$ & $\checkmark$ & $x$ & $\checkmark$ & $\checkmark$ & Deleted \\
\hline 132 & $\begin{array}{c}\text { Expected number of annual hours in which } \\
\text { energy is unserved }\end{array}$ & [17] & $\checkmark$ & $\checkmark$ & $x$ & $x$ & $\checkmark$ & Deleted \\
\hline 133 & Value/level of unserved energy & [17] & $\checkmark$ & $\checkmark$ & $\checkmark$ & $\checkmark$ & $\checkmark$ & Retained \\
\hline 134 & $\begin{array}{c}\text { Energy storage capacity and/or stocks by } \\
\text { fuel and market }\end{array}$ & [17] & $\checkmark$ & $x$ & $\checkmark$ & $\checkmark$ & $\checkmark$ & Deleted \\
\hline 135 & Redundancy in network architecture & [17] & $x$ & $\checkmark$ & $x$ & $x$ & $\checkmark$ & Deleted \\
\hline 136 & $\begin{array}{l}\text { Expected probability of interruption for } \\
\text { long-term planning and design }\end{array}$ & [119] & $x$ & $\checkmark$ & $\checkmark$ & $x$ & $\checkmark$ & Deleted \\
\hline 137 & Expected energy not served per interruption & [119] & $x$ & $\checkmark$ & $\checkmark$ & $x$ & $\checkmark$ & Deleted \\
\hline 138 & $\begin{array}{l}\text { Expected outage duration per interruption } \\
\text { for short-term operational planning }\end{array}$ & [119] & $x$ & $\checkmark$ & $\checkmark$ & $x$ & $\checkmark$ & Deleted \\
\hline 139 & Expected energy loss & [24] & $x$ & $x$ & $\checkmark$ & $x$ & $\checkmark$ & Deleted \\
\hline 140 & Collapse ratio & {$[24]$} & $x$ & $\checkmark$ & $\checkmark$ & $\checkmark$ & $x$ & Deleted \\
\hline 141 & Recovery ratio & {$[24,110]$} & $x$ & $\checkmark$ & $\checkmark$ & $\checkmark$ & $x$ & Deleted \\
\hline 142 & Energy cost stability & [120] & $\checkmark$ & $x$ & $\checkmark$ & $\checkmark$ & $\checkmark$ & Deleted \\
\hline 143 & Stability of energy generation & [120] & $\checkmark$ & $\checkmark$ & $x$ & $x$ & $\checkmark$ & Deleted \\
\hline 144 & Peak load response & [120] & $x$ & $\checkmark$ & $\checkmark$ & $\checkmark$ & $\checkmark$ & Deleted \\
\hline 145 & Market concentration on supply & [120] & $x$ & $\checkmark$ & $x$ & $x$ & $\checkmark$ & Deleted \\
\hline 146 & $\mathrm{CO}_{2} \mathrm{eq}$ emissions & [120] & $x$ & $x$ & $\checkmark$ & $\checkmark$ & $\checkmark$ & Deleted \\
\hline 147 & Fuel use & [120] & $\checkmark$ & $x$ & $\checkmark$ & $\checkmark$ & $\checkmark$ & Deleted \\
\hline 148 & Employment & [120] & $\checkmark$ & $x$ & $\checkmark$ & $\checkmark$ & $\checkmark$ & Deleted \\
\hline 149 & $\begin{array}{l}\text { Levelized costs (incl. capital, } \\
\text { operational/maintenance, fuel costs) }\end{array}$ & [120] & $\checkmark$ & $\checkmark$ & $x$ & $\checkmark$ & $\checkmark$ & Deleted \\
\hline 150 & Technological maturity & [120] & $\checkmark$ & $\checkmark$ & $x$ & $x$ & $\checkmark$ & Deleted \\
\hline 151 & Technological innovation ability & [120] & $\checkmark$ & $\checkmark$ & $x$ & $x$ & $\checkmark$ & Deleted \\
\hline 152 & Energy demand and consumption & {$[8,121]$} & $\checkmark$ & $x$ & $\checkmark$ & $\checkmark$ & $\checkmark$ & Deleted \\
\hline 153 & Flexibility of grid & {$[8,121]$} & $x$ & $\checkmark$ & $x$ & $x$ & $\checkmark$ & Deleted \\
\hline 154 & $\begin{array}{l}\text { Urban energy supply systems for increasing } \\
\text { shares of renewable energy }\end{array}$ & {$[121,122]$} & $\checkmark$ & $x$ & $\checkmark$ & $\checkmark$ & $\checkmark$ & Deleted \\
\hline 155 & Reduced end-use energy demand & {$[111,121,122]$} & $\checkmark$ & $\checkmark$ & $\checkmark$ & $\checkmark$ & $\checkmark$ & Retained \\
\hline 156 & Energy monitoring & {$[8,121]$} & $x$ & $\checkmark$ & $x$ & $x$ & $x$ & Deleted \\
\hline 157 & Reduced reliance on energy & {$[16,62,123-125]$} & $\checkmark$ & $x$ & $\checkmark$ & $\checkmark$ & $\checkmark$ & Deleted \\
\hline 158 & Energy source diversity & $\begin{array}{l}{[16,62,111,123} \\
125-127]\end{array}$ & $\checkmark$ & $x$ & $\checkmark$ & $\checkmark$ & $\checkmark$ & Deleted \\
\hline 159 & Energy storage capabilities & [124-126] & $\checkmark$ & $x$ & $\checkmark$ & $\checkmark$ & $\checkmark$ & Deleted \\
\hline 160 & Redundancy of critical capabilities & {$[62,126,128,129]$} & $\checkmark$ & $\checkmark$ & $x$ & $x$ & $\checkmark$ & Deleted \\
\hline 161 & $\begin{array}{c}\text { Preventative maintenance on energy } \\
\text { systems }\end{array}$ & {$[110,126,129]$} & $x$ & $\checkmark$ & $x$ & $x$ & $\checkmark$ & Deleted \\
\hline 162 & $\begin{array}{l}\text { Sensors, controls, and communication links } \\
\text { to support awareness and response }\end{array}$ & {$[125,126,129]$} & $\checkmark$ & $\checkmark$ & $x$ & $x$ & $x$ & Deleted \\
\hline 163 & Protective measures against external attack & {$[123,126,128]$} & $\checkmark$ & $\checkmark$ & $x$ & $x$ & $\checkmark$ & Deleted \\
\hline 164 & $\begin{array}{c}\text { Design margin to accommodate range of } \\
\text { conditions }\end{array}$ & {$[124,126,129-131]$} & $x$ & $\checkmark$ & $x$ & $x$ & $\checkmark$ & Deleted \\
\hline 165 & $\begin{array}{l}\text { Limited performance degradation under } \\
\text { changing conditions }\end{array}$ & $\begin{array}{c}{[16,124,126,129} \\
130]\end{array}$ & $\checkmark$ & $\checkmark$ & $x$ & $x$ & $\checkmark$ & Deleted \\
\hline 166 & $\begin{array}{l}\text { Operational system protection, e.g., } \\
\text { pressure relief, circuit breakers }\end{array}$ & {$[126,129]$} & $\checkmark$ & $\checkmark$ & $x$ & $x$ & $\checkmark$ & Deleted \\
\hline 167 & Installed/ready redundant components & $\begin{array}{l}{[16,31,49,90,126} \\
128,129,132-135]\end{array}$ & $\checkmark$ & $\checkmark$ & $x$ & $\checkmark$ & $\checkmark$ & Deleted \\
\hline 168 & $\begin{array}{l}\text { Ability to isolate damaged } \\
\text { systems/components (automatic/manual) }\end{array}$ & {$[62,126,129]$} & $\checkmark$ & $\checkmark$ & $x$ & $x$ & $\checkmark$ & Deleted \\
\hline
\end{tabular}


Table A1. Cont.

\begin{tabular}{|c|c|c|c|c|c|c|c|c|}
\hline No. & Primary Index & Ref. & S & U & $\mathbf{F}$ & $\mathbf{O}$ & $\mathbf{R}$ & Result \\
\hline 169 & $\begin{array}{l}\text { Capability for independent } \\
\text { local/subnetwork operation }\end{array}$ & {$[126,128]$} & $\checkmark$ & $\checkmark$ & $x$ & $x$ & $\checkmark$ & Deleted \\
\hline 170 & $\begin{array}{l}\text { System flexibility for reconfiguration } \\
\text { and/or temporary system installation }\end{array}$ & $\begin{array}{c}{[16,125,126,128,} \\
130]\end{array}$ & $\checkmark$ & $\checkmark$ & $x$ & $x$ & $\checkmark$ & Deleted \\
\hline 171 & $\begin{array}{c}\text { Capability to monitor and control portions } \\
\text { of system }\end{array}$ & {$[124,126,129]$} & $\checkmark$ & $\checkmark$ & $x$ & $x$ & $\checkmark$ & Deleted \\
\hline 172 & Fuel flexibility & $\begin{array}{c}{[16,31,62,99,128,} \\
130,136,137]\end{array}$ & $\checkmark$ & $x$ & $\checkmark$ & $x$ & $\checkmark$ & Deleted \\
\hline 173 & $\begin{array}{l}\text { Capability to reroute energy from } \\
\text { available sources }\end{array}$ & {$[16,126,128-130]$} & $\checkmark$ & $\checkmark$ & $x$ & $x$ & $x$ & Deleted \\
\hline 174 & $\begin{array}{l}\text { Investigate and repair malfunctioning } \\
\text { controls or sensors }\end{array}$ & [129] & $x$ & $\checkmark$ & $x$ & $x$ & $\checkmark$ & Deleted \\
\hline 175 & $\begin{array}{l}\text { Energy network flexibility to reestablish } \\
\text { service by priority }\end{array}$ & {$[16,126,129]$} & $\checkmark$ & $\checkmark$ & $x$ & $x$ & $\checkmark$ & Deleted \\
\hline 176 & $\begin{array}{l}\text { Backup communication lighting, power } \\
\text { systems for repair/recovery operations }\end{array}$ & {$[126,129]$} & $\checkmark$ & $\checkmark$ & $x$ & $\checkmark$ & $\checkmark$ & Deleted \\
\hline 177 & $\begin{array}{l}\text { Flexible network architecture to facilitate } \\
\text { modernization and new energy sources }\end{array}$ & {$[16,126,128,130]$} & $\checkmark$ & $\checkmark$ & $x$ & $x$ & $\checkmark$ & Deleted \\
\hline 178 & $\begin{array}{l}\text { visualization capabilities to support system } \\
\text { performance trending }\end{array}$ & {$[62,126,128,129]$} & $\checkmark$ & $\checkmark$ & $x$ & $x$ & $\checkmark$ & Deleted \\
\hline 179 & $\begin{array}{l}\text { Ability to use new/alternative } \\
\text { energy sources }\end{array}$ & {$[16,125,130]$} & $\checkmark$ & $\checkmark$ & $x$ & $x$ & $\checkmark$ & Deleted \\
\hline 180 & $\begin{array}{c}\text { Updating system } \\
\text { configuration/functionality based on } \\
\text { lessons learned }\end{array}$ & {$[16,126,128-130]$} & $\checkmark$ & $\checkmark$ & $x$ & $x$ & $\checkmark$ & Deleted \\
\hline 181 & $\begin{array}{l}\text { Phasing out obsolete or damaged assets and } \\
\text { introducing new assets }\end{array}$ & $\begin{array}{c}{[123,126,128-} \\
130,133,138,139]\end{array}$ & $\checkmark$ & $\checkmark$ & $x$ & $x$ & $\checkmark$ & Deleted \\
\hline 182 & $\begin{array}{l}\text { Integrating new interface standards and } \\
\text { operating system upgrades }\end{array}$ & {$[126,128,129]$} & $\checkmark$ & $x$ & $x$ & $x$ & $\checkmark$ & Deleted \\
\hline 183 & $\begin{array}{l}\text { Updating response equipment/supplies } \\
\text { based on lessons learned }\end{array}$ & [128] & $\checkmark$ & $\checkmark$ & $x$ & $x$ & $\checkmark$ & Deleted \\
\hline 184 & $\begin{array}{l}\text { Capabilities and services prioritized based } \\
\text { on criticality or performance requirements }\end{array}$ & [124] & $\checkmark$ & $\checkmark$ & $x$ & $x$ & $\checkmark$ & Deleted \\
\hline 185 & $\begin{array}{l}\text { Internal and external system } \\
\text { dependencies identified }\end{array}$ & {$[124,125,140]$} & $x$ & $\checkmark$ & $x$ & $x$ & $x$ & Deleted \\
\hline 186 & $\begin{array}{l}\text { Design, control, operational, and } \\
\text { maintenance data archived and protected }\end{array}$ & {$[124,129]$} & $\checkmark$ & $\checkmark$ & $x$ & $\checkmark$ & $\checkmark$ & Deleted \\
\hline 187 & Vendor information available & [124] & $x$ & $\checkmark$ & $x$ & $\checkmark$ & $x$ & Deleted \\
\hline 188 & $\begin{array}{l}\text { Control systems operational and protected } \\
\text { with antivirus and other safeguards }\end{array}$ & {$[124,126,129]$} & $\checkmark$ & $\checkmark$ & $x$ & $x$ & $\checkmark$ & Deleted \\
\hline 189 & $\begin{array}{l}\text { Operating environment forecasts captured } \\
\text { in planning scenarios }\end{array}$ & {$[123,124,126,129]$} & $\checkmark$ & $\checkmark$ & $x$ & $x$ & $x$ & Deleted \\
\hline 190 & $\begin{array}{c}\text { Response/recovery plans established and } \\
\text { distributed }\end{array}$ & {$[124,126,129]$} & $\checkmark$ & $\checkmark$ & $x$ & $x$ & $x$ & Deleted \\
\hline 191 & $\begin{array}{l}\text { Environmental condition forecast and event } \\
\text { warnings broadcast }\end{array}$ & {$[62,125,129]$} & $\checkmark$ & $\checkmark$ & $x$ & $x$ & $\checkmark$ & Deleted \\
\hline 192 & $\begin{array}{l}\text { System status, trends, and margins available } \\
\text { to operators, managers, and customers }\end{array}$ & $\begin{array}{l}{[62,110,125,126,} \\
128,129]\end{array}$ & $\checkmark$ & $\checkmark$ & $x$ & $x$ & $\checkmark$ & Deleted \\
\hline 193 & $\begin{array}{l}\text { Critical system data monitored; } \\
\text { anomalies alarmed }\end{array}$ & {$[62,126,128,129]$} & $\checkmark$ & $x$ & $x$ & $x$ & $\checkmark$ & Deleted \\
\hline 194 & $\begin{array}{l}\text { Operational/troubleshooting/response } \\
\text { procedures available }\end{array}$ & {$[126,129]$} & $\checkmark$ & $\checkmark$ & $x$ & $x$ & $\checkmark$ & Deleted \\
\hline 195 & $\begin{array}{l}\text { Status/trend limits trigger safeguards and } \\
\text { isolate components to stop cascade effect }\end{array}$ & {$[62,125,126]$} & $\checkmark$ & $\checkmark$ & $x$ & $x$ & $\checkmark$ & Deleted \\
\hline 196 & $\begin{array}{l}\text { Status/response/mitigation information } \\
\text { transmitted effectively and efficiently to } \\
\text { stakeholders/decision makers }\end{array}$ & [124] & $\checkmark$ & $\checkmark$ & $x$ & $x$ & $\checkmark$ & Deleted \\
\hline
\end{tabular}


Table A1. Cont.

\begin{tabular}{|c|c|c|c|c|c|c|c|c|}
\hline No. & Primary Index & Ref. & $\mathrm{S}$ & $\mathrm{U}$ & F & $\mathrm{O}$ & $\mathbf{R}$ & Result \\
\hline 197 & $\begin{array}{l}\text { Information and communications } \\
\text { coordinated throughout supply chain }\end{array}$ & [126] & $\checkmark$ & $x$ & $x$ & $x$ & $\checkmark$ & Deleted \\
\hline 198 & $\begin{array}{l}\text { Information available to authorities and } \\
\text { crews regarding customer/community } \\
\text { needs/status }\end{array}$ & {$[128,129]$} & $\checkmark$ & $\checkmark$ & $x$ & $x$ & $\checkmark$ & Deleted \\
\hline 199 & $\begin{array}{l}\text { Recovery progress tracked, synthesized, } \\
\text { and available to decision makers } \\
\text { and stakeholder }\end{array}$ & {$[128,129]$} & $\checkmark$ & $\checkmark$ & $x$ & $x$ & $\checkmark$ & Deleted \\
\hline 200 & $\begin{array}{l}\text { Design, repair parts, and substitution } \\
\text { information available to recovery teams }\end{array}$ & [126] & $\checkmark$ & $\checkmark$ & $x$ & $x$ & $\checkmark$ & Deleted \\
\hline 201 & $\begin{array}{l}\text { Location, availability, and ownership of } \\
\text { energy, hardware, and services for } \\
\text { restoration teams }\end{array}$ & [126] & $\checkmark$ & $\checkmark$ & $x$ & $x$ & $\checkmark$ & Deleted \\
\hline 202 & $\begin{array}{l}\text { Resource needs, sources, and authorities } \\
\text { available to decision makers }\end{array}$ & [128] & $\checkmark$ & $x$ & $x$ & $x$ & $\checkmark$ & Deleted \\
\hline 203 & $\begin{array}{l}\text { Information regarding centralized facilities } \\
\text { and distribution of essential supplies and } \\
\text { services available to community }\end{array}$ & [128] & $\checkmark$ & $x$ & $x$ & $x$ & $\checkmark$ & Deleted \\
\hline 204 & $\begin{array}{l}\text { Coordinating information and } \\
\text { communications among } \\
\text { recovery organizations }\end{array}$ & [128] & $\checkmark$ & $\checkmark$ & $x$ & $x$ & $\checkmark$ & Deleted \\
\hline 205 & $\begin{array}{l}\text { Initiating event, incident point of entry, } \\
\text { and associated vulnerabilities and } \\
\text { impacts identified }\end{array}$ & {$[123,125,126,128,129]$} & $\checkmark$ & $\checkmark$ & $x$ & $x$ & $\checkmark$ & Deleted \\
\hline 206 & $\begin{array}{l}\text { Event data and operating environment } \\
\text { forecasts utilized to anticipate future } \\
\text { conditions/events }\end{array}$ & {$[125,126,128,129]$} & $\checkmark$ & $\checkmark$ & $x$ & $x$ & $\checkmark$ & Deleted \\
\hline 207 & $\begin{array}{l}\text { Updated information about energy } \\
\text { resources, alternatives, and emergent } \\
\text { technologies available to managers } \\
\text { and stakeholders }\end{array}$ & {$[16,125,128,129]$} & $\checkmark$ & $x$ & $x$ & $x$ & $\checkmark$ & Deleted \\
\hline 208 & $\begin{array}{l}\text { Design/operation/maintenance } \\
\text { information updated consistently with } \\
\text { system modifications }\end{array}$ & {$[16,126,129]$} & $\checkmark$ & $\checkmark$ & $x$ & $x$ & $\checkmark$ & Deleted \\
\hline 209 & $\begin{array}{l}\text { Consumer/stakeholder awareness of } \\
\text { energy alternatives, cost/benefits, and } \\
\text { implementation requirements }\end{array}$ & {$[16,124,125]$} & $\checkmark$ & $\checkmark$ & $x$ & $x$ & $\checkmark$ & Deleted \\
\hline 210 & $\begin{array}{l}\text { Community impacts, priorities, } \\
\text { interdependencies updated to capture } \\
\text { lessons learned }\end{array}$ & {$[124,128,129]$} & $\checkmark$ & $\checkmark$ & $x$ & $x$ & $x$ & Deleted \\
\hline 211 & $\begin{array}{l}\text { Response plans updated with } \\
\text { lessons learned }\end{array}$ & {$[125,126,128,129]$} & $\checkmark$ & $\checkmark$ & $x$ & $x$ & $\checkmark$ & Deleted \\
\hline 212 & $\begin{array}{l}\text { Understood performance trade-offs of } \\
\text { organizational goals }\end{array}$ & {$[123,125]$} & $x$ & $\checkmark$ & $x$ & $x$ & $x$ & Deleted \\
\hline 213 & $\begin{array}{l}\text { Broad-based operational and } \\
\text { maintenance training }\end{array}$ & {$[126,129]$} & $x$ & $\checkmark$ & $x$ & $x$ & $\checkmark$ & Deleted \\
\hline 214 & $\begin{array}{l}\text { Periodic operator, management, and } \\
\text { community drills }\end{array}$ & {$[126,128,129]$} & $x$ & $\checkmark$ & $x$ & $x$ & $\checkmark$ & Deleted \\
\hline 215 & $\begin{array}{l}\text { Developed individual expertise in energy } \\
\text { impacts, techniques, and alternatives } \\
\text { (energy-informed culture) }\end{array}$ & [124] & $\checkmark$ & $\checkmark$ & $x$ & $\checkmark$ & $\checkmark$ & Deleted \\
\hline 216 & $\begin{array}{l}\text { Awareness of and focusing of effort on } \\
\text { identified critical assets and services }\end{array}$ & {$[124,126,128]$} & $x$ & $\checkmark$ & $x$ & $x$ & $x$ & Deleted \\
\hline 217 & $\begin{array}{l}\text { Decision-making protocol or aid to } \\
\text { determine proper course of action }\end{array}$ & {$[125,126,128]$} & $x$ & $\checkmark$ & $x$ & $x$ & $\checkmark$ & Deleted \\
\hline 218 & $\begin{array}{l}\text { Operators and managers utilizing critical } \\
\text { thinking and maintain proactive posture to } \\
\text { recognized and arrest events }\end{array}$ & {$[125,126]$} & $\checkmark$ & $\checkmark$ & $x$ & $x$ & $\checkmark$ & Deleted \\
\hline
\end{tabular}


Table A1. Cont.

\begin{tabular}{|c|c|c|c|c|c|c|c|c|}
\hline No. & Primary Index & Ref. & $\mathrm{S}$ & U & $\mathbf{F}$ & $\mathrm{O}$ & $\mathbf{R}$ & Result \\
\hline 219 & $\begin{array}{l}\text { Community response to mitigate impact, } \\
\text { e.g., demand curtailment }\end{array}$ & {$[124,126,128]$} & $\checkmark$ & $\checkmark$ & $x$ & $\checkmark$ & $\checkmark$ & Deleted \\
\hline 220 & $\begin{array}{l}\text { Utilizing data and decision-making aids to } \\
\text { quickly select recovery options }\end{array}$ & [128] & $\checkmark$ & $\checkmark$ & $x$ & $x$ & $\checkmark$ & Deleted \\
\hline 221 & $\begin{array}{l}\text { Recovery crew managing incremental } \\
\text { recovery with available equipment }\end{array}$ & [126] & $\checkmark$ & $\checkmark$ & $x$ & $x$ & $\checkmark$ & Deleted \\
\hline 222 & $\begin{array}{l}\text { Community members utilizing available } \\
\text { resources and improvised to meet } \\
\text { local needs }\end{array}$ & {$[16,124,125,128]$} & $x$ & $\checkmark$ & $x$ & $x$ & $\checkmark$ & Deleted \\
\hline 223 & $\begin{array}{l}\text { Community members managing } \\
\text { constrained energy resources responsibly } \\
\text { and consistent with public guidance }\end{array}$ & {$[16,124,128]$} & $x$ & $\checkmark$ & $x$ & $x$ & $\checkmark$ & Deleted \\
\hline 224 & $\begin{array}{l}\text { Documentation and review of management } \\
\text { response and decision-making processes }\end{array}$ & {$[125,126,128]$} & $x$ & $\checkmark$ & $x$ & $x$ & $x$ & Deleted \\
\hline 225 & $\begin{array}{l}\text { Periodic revisitation of organizational risk } \\
\text { tolerance and mission priorities, adjusting } \\
\text { as necessary }\end{array}$ & {$[124,125]$} & $x$ & $\checkmark$ & $x$ & $x$ & $\checkmark$ & Deleted \\
\hline 226 & $\begin{array}{l}\text { Integration of lessons learned and best } \\
\text { practices from internal and external sources }\end{array}$ & {$[125,126,128,129]$} & $\checkmark$ & $\checkmark$ & $x$ & $x$ & $\checkmark$ & Deleted \\
\hline 227 & $\begin{array}{c}\text { Customers and stakeholders taking action } \\
\text { to implement more resilient } \\
\text { energy solutions }\end{array}$ & {$[16,124-126,129]$} & $\checkmark$ & $x$ & $x$ & $x$ & $\checkmark$ & Deleted \\
\hline 228 & $\begin{array}{c}\text { Identification of stakeholders (internal } \\
\text { and external) }\end{array}$ & {$[126,128]$} & $x$ & $\checkmark$ & $x$ & $x$ & $\checkmark$ & Deleted \\
\hline 229 & $\begin{array}{l}\text { Use of scenario-based war gaming to } \\
\text { develop understanding of system } \\
\text { dependencies and interactions }\end{array}$ & {$[125,126,128,131]$} & $\checkmark$ & $\checkmark$ & $x$ & $x$ & $\checkmark$ & Deleted \\
\hline 230 & $\begin{array}{l}\text { Robust risk analysis and decision support } \\
\text { capabilities to facilitate response }\end{array}$ & {$[123-126,128,129]$} & $\checkmark$ & $x$ & $x$ & $x$ & $\checkmark$ & Deleted \\
\hline 231 & $\begin{array}{l}\text { Decreased overall reliance on energy or } \\
\text { specific sources of energy }\end{array}$ & {$[123,124]$} & $\checkmark$ & $\checkmark$ & $x$ & $\checkmark$ & $\checkmark$ & Deleted \\
\hline 232 & $\begin{array}{c}\text { Priorities and policies established for } \\
\text { event response }\end{array}$ & {$[123-126,128,129]$} & $x$ & $\checkmark$ & $x$ & $x$ & $\checkmark$ & Deleted \\
\hline 233 & $\begin{array}{l}\text { Priorities and operating limits mitigating } \\
\text { disruption to energy needs for key } \\
\text { community functions }\end{array}$ & {$[123,126,128]$} & $x$ & $\checkmark$ & $x$ & $x$ & $\checkmark$ & Deleted \\
\hline 234 & $\begin{array}{l}\text { Predefined protective actions limiting } \\
\text { external influences in physical, } \\
\text { information domains }\end{array}$ & [124-126] & $x$ & $\checkmark$ & $x$ & $x$ & $\checkmark$ & Deleted \\
\hline 235 & $\begin{array}{l}\text { Agile operational management enabling } \\
\text { rapid and effective response under } \\
\text { changing conditions }\end{array}$ & {$[125,126]$} & $\checkmark$ & $\checkmark$ & $x$ & $x$ & $\checkmark$ & Deleted \\
\hline 236 & $\begin{array}{l}\text { Individuals and organizations } \\
\text { implementing response plans }\end{array}$ & {$[124-126,128]$} & $x$ & $x$ & $x$ & $x$ & $\checkmark$ & Deleted \\
\hline 237 & $\begin{array}{l}\text { Individuals and organizations taking } \\
\text { action in response to observations and/or } \\
\text { direction from authorities }\end{array}$ & {$[124,128]$} & $x$ & $\checkmark$ & $x$ & $x$ & $\checkmark$ & Deleted \\
\hline 238 & $\begin{array}{l}\text { Recovery organizations and communities } \\
\text { following contingency recovery plans }\end{array}$ & {$[124,125,128]$} & $\checkmark$ & $\checkmark$ & $x$ & $x$ & $\checkmark$ & Deleted \\
\hline 239 & $\begin{array}{l}\text { Community stakeholders participating in } \\
\text { establishment of energy priorities and } \\
\text { coordination of restoration actions }\end{array}$ & {$[124,126,128]$} & $\checkmark$ & $\checkmark$ & $x$ & $x$ & $\checkmark$ & Deleted \\
\hline 240 & $\begin{array}{l}\text { Shelters and other centralized services } \\
\text { increasing efficiency and control of scarce } \\
\text { energy resources to meet critical needs }\end{array}$ & [126] & $x$ & $x$ & $x$ & $x$ & $x$ & Deleted \\
\hline 241 & $\begin{array}{l}\text { Public/private entities coordinating to } \\
\text { deliver aid to affected parties }\end{array}$ & [128] & $x$ & $\checkmark$ & $x$ & $x$ & $\checkmark$ & Deleted \\
\hline
\end{tabular}


Table A1. Cont.

\begin{tabular}{|c|c|c|c|c|c|c|c|c|}
\hline No. & Primary Index & Ref. & $\mathrm{S}$ & $\mathrm{U}$ & $\mathbf{F}$ & $\mathrm{O}$ & $\mathbf{R}$ & Result \\
\hline 242 & $\begin{array}{l}\text { Proactive neighborhood assistance, } \\
\text { volunteerism, and compliance with energy } \\
\text { response manager direction }\end{array}$ & [128] & $x$ & $\checkmark$ & $x$ & $x$ & $\checkmark$ & Deleted \\
\hline 243 & $\begin{array}{l}\text { Reallocation of human resources to better } \\
\text { address adverse events }\end{array}$ & [128] & $\checkmark$ & $\checkmark$ & $x$ & $x$ & $\checkmark$ & Deleted \\
\hline 244 & $\begin{array}{l}\text { Local governments and stakeholders } \\
\text { staying informed about threats, changing } \\
\text { environment, and protective methods } \\
\text { and technologies }\end{array}$ & {$[123-126,128,129]$} & $\checkmark$ & $\checkmark$ & $x$ & $x$ & $\checkmark$ & Deleted \\
\hline 245 & $\begin{array}{l}\text { Local governments and stakeholders } \\
\text { collaborating to develop, prioritize, and } \\
\text { implement energy portfolio improvement }\end{array}$ & $\begin{array}{c}{[16,123-} \\
126,128,129]\end{array}$ & $\checkmark$ & $\checkmark$ & $x$ & $x$ & $\checkmark$ & Deleted \\
\hline 246 & $\begin{array}{c}\text { Incentives for customers and stakeholders } \\
\text { to implement more resilient } \\
\text { energy solutions }\end{array}$ & $\begin{array}{l}{[16,62,123-} \\
126,128,129]\end{array}$ & $\checkmark$ & $\checkmark$ & $x$ & $x$ & $\checkmark$ & Deleted \\
\hline 247 & $\begin{array}{l}\text { Energy-informed culture leading to } \\
\text { collective decisions and investments which } \\
\text { continually improve energy effectiveness }\end{array}$ & {$[16,62,126,128]$} & $x$ & $\checkmark$ & $x$ & $x$ & $\checkmark$ & Deleted \\
\hline 248 & $\begin{array}{c}\text { Accurate estimation of weather location } \\
\text { and severity }\end{array}$ & {$[57]$} & $\checkmark$ & $x$ & $x$ & $x$ & $\checkmark$ & Deleted \\
\hline 249 & $\begin{array}{l}\text { Energy consciousness of the public } \\
\text { and consumption } \\
\text { behavior/demand-side management }\end{array}$ & $\begin{array}{c}{[8,31,57,69,70,94,99} \\
101,104,113,133,139 \\
141-154]\end{array}$ & $\checkmark$ & $\checkmark$ & $\checkmark$ & $\checkmark$ & $\checkmark$ & Retained \\
\hline 250 & Fast topology reconfiguration & {$[57]$} & $\checkmark$ & $\checkmark$ & $x$ & $x$ & $\checkmark$ & Deleted \\
\hline 251 & $\begin{array}{l}\text { Automated protection and control actions: } \\
\text { load and generation rejection, system } \\
\text { separation, etc. }\end{array}$ & [57] & $\checkmark$ & $\checkmark$ & $x$ & $x$ & $\checkmark$ & Deleted \\
\hline 252 & $\begin{array}{l}\text { Monitoring-development of situation } \\
\text { awareness, advanced visualization and } \\
\text { information systems }\end{array}$ & [57] & $\checkmark$ & $x$ & $x$ & $x$ & $\checkmark$ & Deleted \\
\hline 253 & Ensured communications functionality & [57] & $\checkmark$ & $x$ & $x$ & $x$ & $\checkmark$ & Deleted \\
\hline 254 & Microgrids & {$[57,155,156]$} & $\checkmark$ & $\checkmark$ & $\checkmark$ & $\checkmark$ & $\checkmark$ & Retained \\
\hline 255 & Advanced control and protection schemes & {$[57,110]$} & $\checkmark$ & $x$ & $x$ & $x$ & $\checkmark$ & Deleted \\
\hline 256 & Disaster assessment and priority setting & {$[57]$} & $\checkmark$ & $x$ & $x$ & $x$ & $\checkmark$ & Deleted \\
\hline 257 & $\begin{array}{l}\text { Risk assessment and management for } \\
\text { evaluating and preparing for the risk } \\
\text { introduced by such events }\end{array}$ & {$[57,122]$} & $\checkmark$ & $x$ & $x$ & $x$ & $\checkmark$ & Deleted \\
\hline 258 & Black-start capabilities installed & [57] & $\checkmark$ & $\checkmark$ & $x$ & $x$ & $\checkmark$ & Deleted \\
\hline 259 & Repair crew member mobilization & [57] & $\checkmark$ & $\checkmark$ & $x$ & $x$ & $\checkmark$ & Deleted \\
\hline 260 & $\begin{array}{l}\text { Installation of DER or other onsite } \\
\text { generation units }\end{array}$ & [57] & $\checkmark$ & $\checkmark$ & $x$ & $\checkmark$ & $\checkmark$ & Deleted \\
\hline 261 & $\begin{array}{c}\text { Coordination with adjacent networks, and } \\
\text { repair crews }\end{array}$ & [57] & $\checkmark$ & $\checkmark$ & $x$ & $x$ & $\checkmark$ & Deleted \\
\hline 262 & $\begin{array}{l}\text { Upgrading poles and structures with } \\
\text { stronger, more robust materials }\end{array}$ & [57] & $x$ & $x$ & $x$ & $x$ & $\checkmark$ & Deleted \\
\hline 263 & $\begin{array}{l}\text { Elevating substations and relocating } \\
\text { facilities to areas less prone to flooding }\end{array}$ & [57] & $x$ & $\checkmark$ & $x$ & $x$ & $\checkmark$ & Deleted \\
\hline 264 & $\begin{array}{l}\text { Redundant transmission routes via } \\
\text { additional transmission facilities }\end{array}$ & [57] & $\checkmark$ & $\checkmark$ & $x$ & $x$ & $\checkmark$ & Deleted \\
\hline 265 & $\begin{array}{c}\text { Available energy } \\
\text { sources/generation methods }\end{array}$ & [110] & $\checkmark$ & $x$ & $\checkmark$ & $\checkmark$ & $\checkmark$ & Deleted \\
\hline 266 & $\begin{array}{c}\text { Number of service connections able to } \\
\text { handle entire load }\end{array}$ & [110] & $x$ & $\checkmark$ & $x$ & $x$ & $\checkmark$ & Deleted \\
\hline 267 & Damage assessment methods & [110] & $\checkmark$ & $\checkmark$ & $x$ & $x$ & $\checkmark$ & Deleted \\
\hline 268 & Scenario/contingency planning & [110] & $\checkmark$ & $\checkmark$ & $x$ & $x$ & $\checkmark$ & Deleted \\
\hline 269 & $\begin{array}{c}\text { Local availability of tools/expertise to } \\
\text { address damage }\end{array}$ & [110] & $x$ & $\checkmark$ & $x$ & $x$ & $\checkmark$ & Deleted \\
\hline 270 & Load shedding and load factor & [110] & $\checkmark$ & $\checkmark$ & $x$ & $x$ & $\checkmark$ & Deleted \\
\hline
\end{tabular}


Table A1. Cont.

\begin{tabular}{|c|c|c|c|c|c|c|c|c|}
\hline No. & Primary Index & Ref. & S & U & $\mathbf{F}$ & $\mathbf{O}$ & $\mathbf{R}$ & Result \\
\hline 271 & Estimated lifespan of generation plant & [110] & $\checkmark$ & $\checkmark$ & $x$ & $\checkmark$ & $\checkmark$ & Deleted \\
\hline 272 & $\begin{array}{l}\text { Fortification and robustness } \\
\text { (physical security) }\end{array}$ & $\begin{array}{c}{[62,89,96,98,143,} \\
157-159]\end{array}$ & $\checkmark$ & $x$ & $\checkmark$ & $\checkmark$ & $\checkmark$ & Deleted \\
\hline 273 & $\begin{array}{l}\text { Operational system protection, e.g., system } \\
\text { relief, circuit breakers }\end{array}$ & [31] & $\checkmark$ & $x$ & $x$ & $x$ & $\checkmark$ & Deleted \\
\hline 274 & $\begin{array}{l}\text { Diversification of energy supply-fuel mix, } \\
\text { multisourcing, type of generation }\end{array}$ & $\begin{array}{c}{[16,17,31,62,86-} \\
91,94-108]\end{array}$ & $\checkmark$ & $x$ & $\checkmark$ & $\checkmark$ & $\checkmark$ & Deleted \\
\hline 275 & $\begin{array}{l}\text { Spatially distributed generation (and } \\
\text { critical facilities) }\end{array}$ & $\begin{array}{c}{[31,95,96,99,109,138} \\
139,141,160-163]\end{array}$ & $\checkmark$ & $x$ & $x$ & $x$ & $\checkmark$ & Deleted \\
\hline 276 & $\begin{array}{l}\text { Energy production near point of use } \\
\text { (colocation of supply and demand) }\end{array}$ & {$[96,164,165]$} & $\checkmark$ & $\checkmark$ & $x$ & $x$ & $\checkmark$ & Deleted \\
\hline 277 & $\begin{array}{l}\text { On-site energy production (photovoltaics, } \\
\text { micro-combined heat and power, } \\
\text { trigeneration, thermal panels, small wind } \\
\text { turbines mounted at the corners of the roof) }\end{array}$ & $\begin{array}{l}{[16,70,99,102,147-} \\
150,158,159,161 \\
166-175]\end{array}$ & $\checkmark$ & $x$ & $x$ & $\checkmark$ & $\checkmark$ & Deleted \\
\hline 278 & Solar absorption cooling & {$[176,177]$} & $x$ & $\checkmark$ & $x$ & $\checkmark$ & $\checkmark$ & Deleted \\
\hline 279 & $\begin{array}{l}\text { Large wind turbines located outside the } \\
\text { built-up area }\end{array}$ & {$[162,178,179]$} & $x$ & $\checkmark$ & $x$ & $\checkmark$ & $\checkmark$ & Deleted \\
\hline 280 & Large solar thermal collectors & {$[149,178]$} & $x$ & $\checkmark$ & $x$ & $\checkmark$ & $\checkmark$ & Deleted \\
\hline 281 & $\begin{array}{l}\text { Smart microgrids fed by microturbines and } \\
\text { solar panels (photovoltaics, building } \\
\text { integrated photovoltaics) and storage facilities }\end{array}$ & $\begin{array}{c}{[62,104,109,136,138} \\
141,142,144,151,152 \\
158,180-183]\end{array}$ & $x$ & $\checkmark$ & $x$ & $\checkmark$ & $\checkmark$ & Deleted \\
\hline 282 & $\begin{array}{l}\text { Building-integrated photovoltaic/thermal for } \\
\text { recovery of heat loss form photovoltaics and } \\
\text { building integrated photovoltaics }\end{array}$ & [180] & $x$ & $\checkmark$ & $x$ & $\checkmark$ & $\checkmark$ & Deleted \\
\hline 283 & Ground source heat pumps & {$[149,150,178,184,185]$} & $x$ & $\checkmark$ & $x$ & $\checkmark$ & $\checkmark$ & Deleted \\
\hline 284 & $\begin{array}{l}\text { Waste heat or biomass-fueled combined heat } \\
\text { and power plants }\end{array}$ & {$[138,178,186]$} & $\checkmark$ & $\checkmark$ & $\checkmark$ & $\checkmark$ & $\checkmark$ & Retained \\
\hline 285 & $\begin{array}{l}\text { Biofuel energy (food waste, second generation } \\
\text { cellulosic biofuels, third generation using } \\
\text { algae, etc.) }\end{array}$ & $\begin{array}{c}{[139,182,184,187-} \\
190]\end{array}$ & $\checkmark$ & $\checkmark$ & $\checkmark$ & $\checkmark$ & $\checkmark$ & Retained \\
\hline 286 & Biomass supply chain, wood pellet systems & {$[101,139]$} & $x$ & $\checkmark$ & $x$ & $\checkmark$ & $\checkmark$ & Deleted \\
\hline 287 & $\begin{array}{l}\text { Interdependency and interconnection of } \\
\text { infrastructures and their networks }\end{array}$ & $\begin{array}{c}{[95,96,99,115,159} \\
160,165,191]\end{array}$ & $\checkmark$ & $\checkmark$ & $\checkmark$ & $\checkmark$ & $\checkmark$ & Retained \\
\hline 288 & Regular maintenance & {$[31,33,88,96]$} & $\checkmark$ & $\checkmark$ & $x$ & $\checkmark$ & $\checkmark$ & Deleted \\
\hline 289 & $\begin{array}{l}\text { Generation, transmission, and distribution } \\
\text { efficiency (leakages, etc.) }\end{array}$ & {$[62,86,87,98,192]$} & $\checkmark$ & $x$ & $\checkmark$ & $\checkmark$ & $\checkmark$ & Deleted \\
\hline 290 & Age of the fleet (feeder lines, etc.) & {$[62,193]$} & $x$ & $\checkmark$ & $x$ & $\checkmark$ & $\checkmark$ & Deleted \\
\hline 291 & $\begin{array}{l}\text { Type of feeder lines (overhead/underground } \\
\text { cables; looped/interconnected or } \\
\text { radial configuration) }\end{array}$ & $\begin{array}{l}{[49,95,146,158,159,} \\
\quad 193,194]\end{array}$ & $x$ & $\checkmark$ & $x$ & $\checkmark$ & $\checkmark$ & Deleted \\
\hline 292 & $\begin{array}{l}\text { Natural gas distribution: continuous (grid) vs. } \\
\text { discontinuous (propane tanks) }\end{array}$ & [195] & $x$ & $\checkmark$ & $x$ & $\checkmark$ & $\checkmark$ & Deleted \\
\hline 293 & $\begin{array}{l}\text { Alternative and safer energy sources for } \\
\text { critical infrastructure such as parking gates, } \\
\text { traffic lights, subway, etc. }\end{array}$ & {$[96,191]$} & $\checkmark$ & $\checkmark$ & $x$ & $\checkmark$ & $\checkmark$ & Deleted \\
\hline 294 & $\begin{array}{l}\text { Intelligent ICT infrastructure and } \\
\text { cybersecurity thereof for maintaining } \\
\text { grid operation }\end{array}$ & $\begin{array}{c}{[31,33,49,96,133,158,} \\
191,196,197]\end{array}$ & $\checkmark$ & $\checkmark$ & $x$ & $\checkmark$ & $\checkmark$ & Deleted \\
\hline 295 & Flexible network architecture & [31] & $x$ & $\checkmark$ & $x$ & $x$ & $\checkmark$ & Deleted \\
\hline 296 & $\begin{array}{l}\text { Number of configuration of nodes and links } \\
\text { in the transmission and distribution grid }\end{array}$ & {$[17,22,198]$} & $\checkmark$ & $\checkmark$ & $\checkmark$ & $\checkmark$ & $\checkmark$ & Retained \\
\hline 297 & Backup energy sources and stocks of energy & {$[17,33,96]$} & $\checkmark$ & $x$ & $\checkmark$ & $\checkmark$ & $\checkmark$ & Deleted \\
\hline 298 & $\begin{array}{c}\text { Energy storage facilities involving } \\
\text { electro-chemical batteries, flow batteries, } \\
\text { hydrogen, etc. }\end{array}$ & $\begin{array}{c}{[16,49,70,86,90,109,} \\
138,144,146,199]\end{array}$ & $x$ & $\checkmark$ & $x$ & $\checkmark$ & $\checkmark$ & Deleted \\
\hline 299 & Distributed storage & {$[95,158]$} & $\checkmark$ & $x$ & $x$ & $\checkmark$ & $\checkmark$ & Deleted \\
\hline
\end{tabular}


Table A1. Cont.

\begin{tabular}{|c|c|c|c|c|c|c|c|c|}
\hline No. & Primary Index & Ref. & S & $\mathbf{U}$ & $\mathbf{F}$ & O & $\mathbf{R}$ & Result \\
\hline 300 & $\begin{array}{l}\text { Connectivity of generation and storage } \\
\text { infrastructure }\end{array}$ & {$[88,89,200]$} & $x$ & $\checkmark$ & $x$ & $x$ & $\checkmark$ & Deleted \\
\hline 301 & $\begin{array}{l}\text { Backup data of the utility infrastructure } \\
\text { (information networks, data sharing, etc.) }\end{array}$ & {$[31,157]$} & $x$ & $\checkmark$ & $x$ & $x$ & $\checkmark$ & Deleted \\
\hline 302 & $\begin{array}{l}\text { Spare capacity and reserve margins-resources, } \\
\text { transmission lines, etc. }\end{array}$ & $\begin{array}{l}{[31,49,62,98,100,191} \\
201,202]\end{array}$ & $\checkmark$ & $x$ & $x$ & $x$ & $\checkmark$ & Deleted \\
\hline 303 & $\begin{array}{l}\text { Vehicle-to-grid and vehicle-to-community } \\
\text { selling of surplus power }\end{array}$ & {$[70,150,203]$} & $x$ & $\checkmark$ & $x$ & $x$ & $\checkmark$ & Deleted \\
\hline 304 & $\begin{array}{c}\text { Parks and open space, bioswales, etc. (attention } \\
\text { to regular trimming of trees) }\end{array}$ & {$[193,204-218]$} & $\checkmark$ & $x$ & $\checkmark$ & $\checkmark$ & $\checkmark$ & Deleted \\
\hline 305 & Indigenous (native) vs. invasive plants & {$[138,208]$} & $x$ & $\checkmark$ & $x$ & $\checkmark$ & $x$ & Deleted \\
\hline 306 & Deciduous trees for cold climate & [168] & $x$ & $\checkmark$ & $x$ & $\checkmark$ & $x$ & Deleted \\
\hline 307 & Xeriscape for hot and arid climates & {$[207,219]$} & $x$ & $\checkmark$ & $x$ & $\checkmark$ & $x$ & Deleted \\
\hline 308 & $\begin{array}{c}\text { Urban agriculture (vacant lands, marginal } \\
\text { lands, etc.) }\end{array}$ & [220] & $x$ & $\checkmark$ & $x$ & $x$ & $x$ & Deleted \\
\hline 309 & Green area ration & [213] & $\checkmark$ & $x$ & $\checkmark$ & $\checkmark$ & $\checkmark$ & Deleted \\
\hline 310 & Green wall (vegetative covering, green façade) & {$[213,221-223]$} & $x$ & $\checkmark$ & $x$ & $x$ & $x$ & Deleted \\
\hline 311 & Green roof (living roof) & $\begin{array}{l}{[138,206,215,219,} \\
224-227]\end{array}$ & $x$ & $\checkmark$ & $x$ & $x$ & $x$ & Deleted \\
\hline 312 & $\begin{array}{c}\text { Rainwater harvesting, decentralized water } \\
\text { harvesting systems }\end{array}$ & {$[137,147,204,228]$} & $x$ & $\checkmark$ & $x$ & $x$ & $x$ & Deleted \\
\hline 313 & Water conservation & {$[147,219]$} & $x$ & $\checkmark$ & $x$ & $x$ & $x$ & Deleted \\
\hline 314 & $\begin{array}{l}\text { Heat recovery and energy generation } \\
\text { from sewage }\end{array}$ & {$[204,229]$} & $x$ & $\checkmark$ & $x$ & $\checkmark$ & $\checkmark$ & Deleted \\
\hline 315 & $\begin{array}{c}\text { Separation of used water into grey and } \\
\text { black flows }\end{array}$ & [219] & $x$ & $\checkmark$ & $x$ & $\checkmark$ & $x$ & Deleted \\
\hline 316 & $\begin{array}{l}\text { Removing and recovering ammonium and } \\
\text { phosphate from wastewater }\end{array}$ & [219] & $x$ & $\checkmark$ & $x$ & $\checkmark$ & $x$ & Deleted \\
\hline 317 & Waterscape as a natural heat sink & {$[209,215,230]$} & $x$ & $\checkmark$ & $x$ & $x$ & $x$ & Deleted \\
\hline 318 & Roof ponds & $\begin{array}{l}{[99,122,136,231]} \\
{[113,115,139,148}\end{array}$ & $x$ & $\checkmark$ & $x$ & $x$ & $x$ & Deleted \\
\hline 319 & Redesign and refurbishment (retrofit) & $\begin{array}{c}149,151,164,207,219, \\
232-235]\end{array}$ & $x$ & $\checkmark$ & $x$ & $x$ & $x$ & Deleted \\
\hline 320 & Glazing & $\begin{array}{c}149,151,164,207,219 \\
232-235]\end{array}$ & $x$ & $\checkmark$ & $x$ & $x$ & $x$ & Deleted \\
\hline 321 & Net zero- and net positive-energy buildings & {$[148,163,235,236]$} & $\checkmark$ & $\checkmark$ & $x$ & $x$ & $\checkmark$ & Deleted \\
\hline 322 & Insulation and dynamic insulation of buildings & $\begin{array}{c}{[104,109,139,141,} \\
147-149,152,153, \\
159,168,176,214,219, \\
233,235,237-239]\end{array}$ & $x$ & $\checkmark$ & $x$ & $x$ & $\checkmark$ & Deleted \\
\hline 323 & Cut-off air conditioning waste heat discharge & [223] & $x$ & $\checkmark$ & $x$ & $x$ & $x$ & Deleted \\
\hline 324 & Net zero-energy neighborhoods & [148] & $x$ & $\checkmark$ & $x$ & $x$ & $\checkmark$ & Deleted \\
\hline 325 & $\begin{array}{l}\text { Pooling of the built environment (shared walls) } \\
\text { District energy systems-using }\end{array}$ & {$[148,217]$} & $x$ & $\checkmark$ & $x$ & $x$ & $\checkmark$ & Deleted \\
\hline 326 & $\begin{array}{l}\text { low-temperature heat from renewable sources } \\
\text { and industrial waste heat }\end{array}$ & {$[87,137,138,151,184]$} & $\checkmark$ & $\checkmark$ & $x$ & $x$ & $\checkmark$ & Deleted \\
\hline 327 & Infrastructure for active transportation modes & $\begin{array}{l}{[136,138,164,168,} \\
196,220,240-244]\end{array}$ & $x$ & $\checkmark$ & $x$ & $x$ & $x$ & Deleted \\
\hline 328 & Modal split & {$[87,241]$} & $x$ & $\checkmark$ & $x$ & $x$ & $x$ & Deleted \\
\hline 329 & Size of cars & [196] & $x$ & $\checkmark$ & $x$ & $x$ & $x$ & Deleted \\
\hline 330 & Fuel efficiency of cars & {$[115,196,243]$} & $x$ & $\checkmark$ & $x$ & $x$ & $x$ & Deleted \\
\hline 331 & $\begin{array}{l}\text { Supporting promotion of hybrid vehicles and } \\
\text { installing electric vehicle plug-ins in locations } \\
\text { where multiple use can be achieved }\end{array}$ & {$[31,70,99,136-138]$} & $\checkmark$ & $\checkmark$ & $\checkmark$ & $\checkmark$ & $\checkmark$ & Retained \\
\hline
\end{tabular}


Table A1. Cont.

\begin{tabular}{|c|c|c|c|c|c|c|c|c|}
\hline No. & Primary Index & Ref. & S & U & $\mathbf{F}$ & $\mathbf{O}$ & $\mathbf{R}$ & Result \\
\hline 332 & $\begin{array}{l}\text { Enhancing energy efficiency through } \\
\text { innovation and technology (building, } \\
\text { industry, transportation) }\end{array}$ & $\begin{array}{c}331,62,69,94,96,99, \\
117,143,144,147,150 \\
164,165,180,184,186, \\
228,237,241,243,245]\end{array}$ & $\checkmark$ & $\checkmark$ & $x$ & $x$ & $\checkmark$ & Deleted \\
\hline 333 & Energy conservation & [139] & $\checkmark$ & $x$ & $\checkmark$ & $\checkmark$ & $\checkmark$ & Deleted \\
\hline 334 & Energy self sufficiency & {$[91,99,160]$} & $x$ & $\checkmark$ & $\checkmark$ & $\checkmark$ & $x$ & Deleted \\
\hline 335 & Energy cycling & {$[70,142]$} & $x$ & $\checkmark$ & $x$ & $x$ & $\checkmark$ & Deleted \\
\hline 336 & Waste management and waste incineration & {$[86,108,147,184]$} & $\checkmark$ & $x$ & $\checkmark$ & $\checkmark$ & $\checkmark$ & Deleted \\
\hline 337 & $\begin{array}{l}\text { Environmental and socioeconomic impacts of } \\
\text { energy system }\end{array}$ & {$[86,98,99,108]$} & $x$ & $\checkmark$ & $x$ & $x$ & $\checkmark$ & Deleted \\
\hline 338 & $\begin{array}{l}\text { Reducing energy footprint of water } \\
\text { production, treatment, and distribution }\end{array}$ & $\begin{array}{l}{[95,116,138,192,228} \\
229,246,247]\end{array}$ & $x$ & $\checkmark$ & $x$ & $x$ & $\checkmark$ & Deleted \\
\hline 339 & $\begin{array}{l}\text { Provision of less energy-intensive rainwater } \\
\text { harvesting systems in buildings }\end{array}$ & [228] & $x$ & $\checkmark$ & $x$ & $x$ & $\checkmark$ & Deleted \\
\hline 340 & Water and energy resource coupling & [109] & $x$ & $\checkmark$ & $x$ & $x$ & $\checkmark$ & Deleted \\
\hline 341 & $\begin{array}{l}\text { Reducing energy footprint of wastewater } \\
\text { collection, treatment, and discharge }\end{array}$ & [138] & $x$ & $\checkmark$ & $x$ & $x$ & $\checkmark$ & Deleted \\
\hline 342 & $\begin{array}{l}\text { Reducing water footprint of energy production } \\
\text { and transmission }\end{array}$ & {$[95,116,192,246,247]$} & $\checkmark$ & $\checkmark$ & $x$ & $x$ & $\checkmark$ & Deleted \\
\hline 343 & $\begin{array}{l}\text { Improving the efficiency of energy production } \\
\text { by enhancing water quality }\end{array}$ & [187] & $\checkmark$ & $\checkmark$ & $x$ & $x$ & $\checkmark$ & Deleted \\
\hline 344 & $\begin{array}{l}\text { Understanding the water intensity of fuels } \\
\text { used for electricity generation }\end{array}$ & [247] & $x$ & $\checkmark$ & $x$ & $x$ & $\checkmark$ & Deleted \\
\hline 345 & $\begin{array}{l}\text { Less water-intensive technologies for cooling } \\
\text { purposes in thermoelectric plants }\end{array}$ & {$[95,192,246]$} & $x$ & $\checkmark$ & $x$ & $x$ & $\checkmark$ & Deleted \\
\hline 346 & $\begin{array}{l}\text { Use of natural gas for steamed turbines and } \\
\text { combined cycle plants }\end{array}$ & {$[192,246]$} & $\checkmark$ & $\checkmark$ & $x$ & $x$ & $\checkmark$ & Deleted \\
\hline 347 & $\begin{array}{l}\text { Use of wet cooling towers instead of } \\
\text { once-through cooling }\end{array}$ & [246] & $\checkmark$ & $\checkmark$ & $x$ & $x$ & $\checkmark$ & Deleted \\
\hline 348 & $\begin{array}{l}\text { Knowing groundwater implications of energy } \\
\text { (technologies, extraction, etc.) }\end{array}$ & {$[86,187,229]$} & $x$ & $\checkmark$ & $x$ & $x$ & $\checkmark$ & Deleted \\
\hline 349 & $\begin{array}{l}\text { Scenario-based energy planning and } \\
\text { risk management }\end{array}$ & {$[31,133,229]$} & $x$ & $x$ & $x$ & $x$ & $\checkmark$ & Deleted \\
\hline 350 & $\begin{array}{l}\text { Risk communication and energy response of } \\
\text { urban governance }\end{array}$ & [96] & $x$ & $x$ & $x$ & $x$ & $\checkmark$ & Deleted \\
\hline 351 & $\begin{array}{l}\text { Community involvement in and/or ownership } \\
\text { of renewable energy generation }\end{array}$ & [96] & $\checkmark$ & $\checkmark$ & $x$ & $x$ & $\checkmark$ & Deleted \\
\hline 352 & $\begin{array}{l}\text { Institutional coordination on water, food, } \\
\text { health, and energy nexus }\end{array}$ & [116] & $\checkmark$ & $\checkmark$ & $x$ & $x$ & $\checkmark$ & Deleted \\
\hline 353 & Reliance on nuclear energy & {$[31,154]$} & $\checkmark$ & $\checkmark$ & $\checkmark$ & $\checkmark$ & $\checkmark$ & Retained \\
\hline 354 & $\begin{array}{l}\text { Regular publication of energy planning } \\
\text { documents and statistics }\end{array}$ & [99] & $x$ & $\checkmark$ & $x$ & $x$ & $\checkmark$ & Deleted \\
\hline 355 & $\begin{array}{l}\text { Market competitiveness and investment risk of } \\
\text { decentralized renewable energy }\end{array}$ & {$[99,139,150,239]$} & $x$ & $\checkmark$ & $x$ & $x$ & $\checkmark$ & Deleted \\
\hline 356 & $\begin{array}{l}\text { Requirement for suppliers to source a } \\
\text { proportion of electricity from renewables }\end{array}$ & [239] & $x$ & $\checkmark$ & $x$ & $x$ & $\checkmark$ & Deleted \\
\hline 357 & $\begin{array}{l}\text { Legal and regulatory frameworks to encourage } \\
\text { technological development and transition } \\
\text { towards energy resilience }\end{array}$ & {$[161,180,248]$} & $x$ & $\checkmark$ & $x$ & $x$ & $\checkmark$ & Deleted \\
\hline 358 & Measures against electricity theft & [249] & $x$ & $\checkmark$ & $x$ & $x$ & $\checkmark$ & Deleted \\
\hline 359 & $\begin{array}{l}\text { Attracting private sector's investment in } \\
\text { low-carbon development }\end{array}$ & {$[95,115-117]$} & $x$ & $\checkmark$ & $x$ & $x$ & $\checkmark$ & Deleted \\
\hline 360 & $\begin{array}{l}\text { Financial and nonfinancial mechanisms and } \\
\text { incentives for promoting green products and } \\
\text { renewable energy technologies and } \\
\text { enhancing affordability }\end{array}$ & {$[95,115-117]$} & $x$ & $\checkmark$ & $x$ & $x$ & $\checkmark$ & Deleted \\
\hline
\end{tabular}


Table A2. Aggregated index selection for CI.

\begin{tabular}{|c|c|c|c|c|c|c|c|c|}
\hline No. & Primary Index & Ref. & $\mathbf{S}$ & $\mathbf{U}$ & $\mathbf{F}$ & $\mathbf{O}$ & $\mathbf{R}$ & Result \\
\hline 1 & Train transportation & [250] & $\checkmark$ & $\checkmark$ & $\checkmark$ & $\checkmark$ & $\checkmark$ & Retained \\
\hline 2 & $\begin{array}{l}\text { Emergency organization and infrastructure in } \\
\text { place and critical functions identified }\end{array}$ & {$[44,118]$} & $\checkmark$ & $\checkmark$ & $x$ & $x$ & $\checkmark$ & Deleted \\
\hline 3 & Waste and disposal & $\begin{array}{l}{[41,120,} \\
122]\end{array}$ & $\checkmark$ & $\checkmark$ & $\checkmark$ & $\checkmark$ & $\checkmark$ & Retained \\
\hline 4 & Land use requirement & [120] & $x$ & $\checkmark$ & $x$ & $x$ & $\checkmark$ & Deleted \\
\hline 5 & Level of public resistance/opposition & [120] & $x$ & $\checkmark$ & $x$ & $x$ & $x$ & Deleted \\
\hline 6 & Market size-domestic/potential export & [120] & $\checkmark$ & $\checkmark$ & $x$ & $x$ & $\checkmark$ & Deleted \\
\hline 7 & Permeable pavement and bioswales & [121] & $\checkmark$ & $\checkmark$ & $x$ & $x$ & $\checkmark$ & Deleted \\
\hline 8 & Urban tree canopy & [121] & $x$ & $\checkmark$ & $x$ & $x$ & $x$ & Deleted \\
\hline 9 & Water demand and consumption & $\begin{array}{l}{[8,121,122,} \\
251,252]\end{array}$ & $\checkmark$ & $\checkmark$ & $\checkmark$ & $\checkmark$ & $\checkmark$ & Retained \\
\hline 10 & Water-efficient landscaping & {$[8,41,121]$} & $x$ & $\checkmark$ & $x$ & $x$ & $x$ & Deleted \\
\hline 11 & Protection of water-sensitive lands & [121] & $\checkmark$ & $\checkmark$ & $\checkmark$ & $x$ & $x$ & Deleted \\
\hline 13 & Water quality and quantity monitoring & {$[121,252]$} & $x$ & $\checkmark$ & $\checkmark$ & $\checkmark$ & $x$ & Deleted \\
\hline 14 & High-efficiency irrigation & {$[8,121]$} & $x$ & $\checkmark$ & $\checkmark$ & $\checkmark$ & $x$ & Deleted \\
\hline 15 & $\begin{array}{l}\text { High-frequency schedule for } \\
\text { public transportation }\end{array}$ & {$[41,42,121]$} & $x$ & $\checkmark$ & $\checkmark$ & $x$ & $x$ & Deleted \\
\hline 16 & Principle arterial miles per square mile & [121] & $X$ & $\checkmark$ & $\checkmark$ & $x$ & $x$ & Deleted \\
\hline 17 & Vehicle ownership & $\begin{array}{l}{[8,10,121} \\
251,253]\end{array}$ & $\checkmark$ & $\checkmark$ & $\checkmark$ & $\checkmark$ & $\checkmark$ & Retained \\
\hline 18 & Parks & {$[8,121]$} & $x$ & $\checkmark$ & $\checkmark$ & $\checkmark$ & $x$ & Deleted \\
\hline 19 & Forest conservation & {$[8,121]$} & $x$ & $\checkmark$ & $\checkmark$ & $x$ & $x$ & Deleted \\
\hline 20 & Waste management & {$[8,121]$} & $\checkmark$ & $x$ & $\checkmark$ & $\checkmark$ & $\checkmark$ & Deleted \\
\hline 21 & Provision of open space for shelter & {$[8,121,122]$} & $\checkmark$ & $\checkmark$ & $\checkmark$ & $\checkmark$ & $\checkmark$ & Retained \\
\hline 22 & Percentage of vacant rental units & [121] & $x$ & $\checkmark$ & $x$ & $\checkmark$ & $x$ & Deleted \\
\hline 23 & Number of hotels/motels per square mile & {$[8,121]$} & $x$ & $\checkmark$ & $\checkmark$ & $\checkmark$ & $x$ & Deleted \\
\hline 24 & Evacuation route & {$[8,121]$} & $x$ & $\checkmark$ & $\checkmark$ & $x$ & $x$ & Deleted \\
\hline 25 & Building insulation, layout, and orientation & [121] & $x$ & $\checkmark$ & $x$ & $\checkmark$ & $x$ & Deleted \\
\hline 26 & Reducing air infiltration and thermal bridging & [121] & $x$ & $\checkmark$ & $x$ & $\checkmark$ & $\checkmark$ & Deleted \\
\hline 27 & Natural ventilation & [121] & $x$ & $\checkmark$ & $x$ & $x$ & $x$ & Deleted \\
\hline 28 & Preservation of housing & [121] & $x$ & $\checkmark$ & $x$ & $x$ & $x$ & Deleted \\
\hline 29 & Building codes & [121] & $x$ & $\checkmark$ & $\checkmark$ & $\checkmark$ & $x$ & Deleted \\
\hline 30 & Housing age & [121] & $x$ & $\checkmark$ & $\checkmark$ & $\checkmark$ & $x$ & Deleted \\
\hline 31 & Generating and making use of information & [121] & $\checkmark$ & $\checkmark$ & $x$ & $x$ & $\checkmark$ & Deleted \\
\hline 32 & $\begin{array}{l}\text { Geospatial information and communication } \\
\text { technology }\end{array}$ & [121] & $\checkmark$ & $\checkmark$ & $x$ & $x$ & $\checkmark$ & Deleted \\
\hline 33 & Volunteered geographic information & [121] & $x$ & $\checkmark$ & $x$ & $x$ & $\checkmark$ & Deleted \\
\hline 34 & Visualization technologies & [121] & $x$ & $\checkmark$ & $x$ & $x$ & $\checkmark$ & Deleted \\
\hline 35 & Alerts and emergency notification systems & [121] & $\checkmark$ & $\checkmark$ & $x$ & $x$ & $\checkmark$ & Deleted \\
\hline 36 & Embracing e-commerce & [121] & $x$ & $\checkmark$ & $x$ & $x$ & $\checkmark$ & Deleted \\
\hline 37 & Biodiversity & {$[8,121]$} & $x$ & $\checkmark$ & $x$ & $x$ & $\checkmark$ & Deleted \\
\hline 38 & Restoration of hydrologic flows & {$[8,121]$} & $x$ & $\checkmark$ & $x$ & $x$ & $\checkmark$ & Deleted \\
\hline 39 & Conservation of ecologically vulnerable areas & {$[121,254]$} & $x$ & $\checkmark$ & $x$ & $x$ & $\checkmark$ & Deleted \\
\hline 40 & Proximity of different habitats & [121] & $x$ & $\checkmark$ & $x$ & $x$ & $\checkmark$ & Deleted \\
\hline 41 & Erosion rates & [121] & $\checkmark$ & $\checkmark$ & $x$ & $x$ & $\checkmark$ & Deleted \\
\hline 42 & Urban green commons & {$[121,122]$} & $\checkmark$ & $\checkmark$ & $\checkmark$ & $\checkmark$ & $\checkmark$ & Retained \\
\hline 43 & Culture of cooperation & [121] & $x$ & $\checkmark$ & $x$ & $x$ & $x$ & Deleted \\
\hline 44 & Balance demographic distribution & [121] & $x$ & $x$ & $\checkmark$ & $\checkmark$ & $\checkmark$ & Deleted \\
\hline 45 & Aging population & [121] & $x$ & $\checkmark$ & $x$ & $x$ & $\checkmark$ & Deleted \\
\hline 46 & Responsive health systems & [121] & $\checkmark$ & $\checkmark$ & $\checkmark$ & $\checkmark$ & $\checkmark$ & Retained \\
\hline 47 & Health coverage and access & {$[8,121,253]$} & $\checkmark$ & $\checkmark$ & $\checkmark$ & $\checkmark$ & $\checkmark$ & Retained \\
\hline 48 & Road density & {$[10,45,251]$} & $\checkmark$ & $\checkmark$ & $\checkmark$ & $\checkmark$ & $\checkmark$ & Retained \\
\hline 49 & Distribution of fire stations & [45] & $\checkmark$ & $\checkmark$ & $x$ & $\checkmark$ & $\checkmark$ & Deleted \\
\hline 50 & Distribution of police stations & {$[45]$} & $\checkmark$ & $\checkmark$ & $x$ & $\checkmark$ & $\checkmark$ & Deleted \\
\hline
\end{tabular}


Table A2. Cont.

\begin{tabular}{ccccccccc}
\hline No. & Primary Index & Ref. & S & U & F & O & R & Result \\
\hline 51 & Distribution of civil air defense facilities & {$[45]$} & $\checkmark$ & $\checkmark$ & $X$ & $\checkmark$ & $\checkmark$ & Deleted \\
52 & Distribution of emergency shelters & {$[45]$} & $\checkmark$ & $\checkmark$ & $X$ & $\checkmark$ & $\checkmark$ & Deleted \\
53 & Land types & {$[45]$} & $X$ & $\checkmark$ & $X$ & $X$ & $X$ & Deleted \\
54 & College students & {$[251]$} & $X$ & $\checkmark$ & $\checkmark$ & $\checkmark$ & $\checkmark$ & Deleted \\
55 & Hospital distribution & {$[10,45]$} & $\checkmark$ & $\checkmark$ & $\checkmark$ & $\checkmark$ & $\checkmark$ & Retained \\
56 & Medical rescue capability & {$[10,45,251]$} & $\checkmark$ & $\checkmark$ & $\checkmark$ & $\checkmark$ & $\checkmark$ & Retained \\
57 & Ecological restoration capacity-green & {$[10,45,251]$} & $\checkmark$ & $\checkmark$ & $\checkmark$ & $\checkmark$ & $\checkmark$ & Retained \\
58 & coverage ratio & {$[45]$} & $\checkmark$ & $\checkmark$ & $\checkmark$ & $\checkmark$ & $\checkmark$ & Retained \\
59 & Social security & {$[10]$} & $\checkmark$ & $\checkmark$ & $\checkmark$ & $\checkmark$ & $\checkmark$ & Retained \\
60 & Gas supply pipeline & {$[10,41]$} & $\checkmark$ & $\checkmark$ & $\checkmark$ & $\checkmark$ & $\checkmark$ & Retained \\
61 & Drainage pipeline & {$[10,251]$} & $\checkmark$ & $\checkmark$ & $\checkmark$ & $\checkmark$ & $\checkmark$ & Retained \\
62 & Internet users & {$[41,251,253]$} & $\checkmark$ & $\checkmark$ & $\checkmark$ & $\checkmark$ & $\checkmark$ & Retained \\
63 & Mobile phone users & {$[251,253]$} & $\checkmark$ & $\checkmark$ & $\checkmark$ & $\checkmark$ & $\checkmark$ & Retained \\
64 & Medical insurance coverage & {$[251]$} & $X$ & $\checkmark$ & $\checkmark$ & $\checkmark$ & $X$ & Deleted \\
\hline
\end{tabular}

Table A3. Aggregated index selection for CV.

\begin{tabular}{|c|c|c|c|c|c|c|c|c|}
\hline No. & Primary Index & Ref. & $S$ & $\mathrm{U}$ & $\mathbf{F}$ & $\mathrm{O}$ & $\mathbf{R}$ & Result \\
\hline 1 & $\begin{array}{l}\text { Human health impact-the degree to which a } \\
\text { disruption in the system might feasibly harm } \\
\text { the health of employees or the public }\end{array}$ & [52] & $\checkmark$ & $\checkmark$ & $\checkmark$ & $\checkmark$ & $\checkmark$ & Retained \\
\hline 2 & Electricity consumption per capita & [112] & $\checkmark$ & $\checkmark$ & $\checkmark$ & $\checkmark$ & $\checkmark$ & Retained \\
\hline 3 & Climate resilience & [120] & $x$ & $\checkmark$ & $x$ & $x$ & $\checkmark$ & Deleted \\
\hline 4 & Noise pollution & [120] & $x$ & $\checkmark$ & $x$ & $\checkmark$ & $\checkmark$ & Deleted \\
\hline 5 & Aesthetic/functional impact & [120] & $x$ & $\checkmark$ & $x$ & $x$ & $\checkmark$ & Deleted \\
\hline 6 & Mortality and morbidity due to air pollution & [120] & $x$ & $\checkmark$ & $x$ & $x$ & $\checkmark$ & Deleted \\
\hline 7 & Accident fatalities & [120] & $x$ & $\checkmark$ & $\checkmark$ & $\checkmark$ & $\checkmark$ & Deleted \\
\hline 8 & $\begin{array}{l}\text { Ecosystem damages due to acidification and } \\
\text { eutrophication caused by pollution from } \\
\text { electricity production }\end{array}$ & [120] & $x$ & $\checkmark$ & $x$ & $x$ & $\checkmark$ & Deleted \\
\hline 9 & Seismic risk & [45] & $x$ & $\checkmark$ & $\checkmark$ & $\checkmark$ & $\checkmark$ & Deleted \\
\hline 10 & Flood risk & {$[45,122]$} & $x$ & $\checkmark$ & $\checkmark$ & $\checkmark$ & $\checkmark$ & Deleted \\
\hline 11 & Meteorological hazard & [45] & $x$ & $\checkmark$ & $\checkmark$ & $\checkmark$ & $\checkmark$ & Deleted \\
\hline 12 & Geological hazard risk & [45] & $x$ & $\checkmark$ & $\checkmark$ & $\checkmark$ & $\checkmark$ & Deleted \\
\hline 13 & Hazard of industrial disaster & [45] & $x$ & $\checkmark$ & $x$ & $x$ & $\checkmark$ & Deleted \\
\hline 14 & Population density & {$[45,251]$} & $\checkmark$ & $\checkmark$ & $\checkmark$ & $\checkmark$ & $\checkmark$ & Retained \\
\hline 15 & Demographic structure & $\begin{array}{c}{[45,251,} \\
253]\end{array}$ & $\checkmark$ & $\checkmark$ & $\checkmark$ & $\checkmark$ & $\checkmark$ & Retained \\
\hline 16 & Demographic change & {$[45,251]$} & $\checkmark$ & $\checkmark$ & $\checkmark$ & $\checkmark$ & $\checkmark$ & Retained \\
\hline 17 & Distribution of important buildings & [45] & $\checkmark$ & $\checkmark$ & $\checkmark$ & $\checkmark$ & $\checkmark$ & Retained \\
\hline 18 & GDP per capita & {$[10,45,251]$} & $\checkmark$ & $\checkmark$ & $\checkmark$ & $\checkmark$ & $\checkmark$ & Retained \\
\hline 19 & Affected elements and components & [110] & $x$ & $\checkmark$ & $\checkmark$ & $\checkmark$ & $\checkmark$ & Deleted \\
\hline 20 & Number of households affected & [110] & $x$ & $\checkmark$ & $\checkmark$ & $\checkmark$ & $\checkmark$ & Deleted \\
\hline
\end{tabular}

\section{References}

1. Foreign Ministry Spokesperson Wang Wenbin's Regular Press Conference on 23 September, in the State Council Information Office of the People's Republic of China. Available online: http:/ / www.scio.gov.cn (accessed on 1 February 2022).

2. Elmqvist, T.; Andersson, E.; Frantzeskaki, N.; McPhearson, T.; Olsson, P.; Gaffney, O.; Takeuchi, K.; Folke, C. Sustainability and resilience for transformation in the urban century. Nat. Sustain. 2019, 2, 267-273. [CrossRef]

3. Presidential Policy Directive-Critical Infrastructure Security and Resilience. In White House. Available online: http://www. whitehouse.gov/the-press-office/2013/02/12/presidential-policy-directive-criticalinfrastructure-security-and-resil (accessed on 1 February 2022). 
4. Gholami, A.; Shekari, T.; Amirioun, M.H.; Aminifar, F.; Amini, M.H.; Sargolzaei, A. Toward a Consensus on the Definition and Taxonomy of Power System Resilience. IEEE Access 2018, 6, 32035-32053. [CrossRef]

5. Baik, S.; Davis, A.L.; Park, J.W.; Sirinterlikci, S.; Morgan, M.G. Estimating what US residential customers are willing to pay for resilience to large electricity outages of long duration. Nat. Energy 2020, 5, 250-258. [CrossRef]

6. Jones, L.; Constas, A.M.; Matthews, N.; Verkaart, S. Advancing resilience measurement. Nat. Sustain. 2021, 4, 288-289. [CrossRef]

7. Desouza, K.; Flanery, T.H. Designing, planning, and managing resilient cities: A conceptual framework. Cities 2013, 35, 89-99. [CrossRef]

8. Zang, X.; Wang, Q. The Evolution of the Urban Resilience Concept, and Its Research Contents and Development Trend. Sci. Technol. Rev. 2019, 37, 11.

9. Li, G.; Xu, B. Measurement and improvement of urban resilience in China. J. Shandong Univ. Sci. Technol. 2018, 20, 8.

10. Zhang, M.; Feng, X. Comprehensive resilience evaluation of cities in China. Urban Probl. 2018, 10, 10.

11. Sharifi, A. Urban Resilience Assessment: Mapping Knowledge Structure and Trends. Sustainability 2020, 12, 5918. [CrossRef]

12. Zhao, P.; Chapman, R.; Randal, E.; Howden-Chapman, P. Understanding Resilient Urban Futures: A Systemic Modelling Approach. Sustainability 2013, 5, 3202-3223. [CrossRef]

13. Wang, L.; Xue, X.; Zhang, Y.; Luo, X. Exploring the Emerging Evolution Trends of Urban Resilience Research by Scientometric Analysis. Int. J. Environ. Res. Public Health 2018, 15, 2181. [CrossRef] [PubMed]

14. Afgan, N.; Veziroglu, A. Sustainable resilience of hydrogen energy system. Int. J. Hydrog. Energy 2012, 37, 5461-5467. [CrossRef]

15. Sharifi, A.; Yamagata, Y. Principles and criteria for assessing urban energy resilience: A literature review. Renew. Sustain. Energy Rev. 2016, 60, 1654-1677. [CrossRef]

16. O'Brien, G.; Hope, A. Localism and energy: Negotiating approaches to embedding resilience in energy systems. Energy Policy 2010, 38, 7550-7558. [CrossRef]

17. Chaudry, M.; Ekins, P.; Ramachandran, K.; Shakoor, A.; Skea, J.; Strbac, G.; Wang, X.; Whitaker, J. Building a Resilient UK Energy System; UK Energy Research Centre: London, UK, 2011; p. 120.

18. Erker, S.; Stangl, R.; Stoeglehner, G. Resilience in the light of energy crises-Part II: Application of the regional energy resilience assessment. J. Clean. Prod. 2017, 164, 495-507. [CrossRef]

19. Wang, H.; Fang, J.; He, J.; Mo, W.; Li, H.; Qin, Y.; Miao, X. A Review of Resilient Distribution Network under Extreme Disasters. Distrib. Util. 2019, 10, 20-29. [CrossRef]

20. Shetyy, S.; Krishnappa, B.; Nikol, D.M. Cyber Resilience Metrics for Bulk Power Systems. Industrial Control Systems Joint Working Group (ICSJWG) Quarterly Newsletter. 2017, p. 5. Available online: https:/ / cred-c.org/publications/cyber-resilience-metrics-bulkpower-systems (accessed on 1 February 2022).

21. Lin, Y.; Bie, Z. Study on the Resilience of the Integrated Energy System. Energy Procedia 2016, 103, 171-176. [CrossRef]

22. Ouyang, M.; Dueñas-Osorio, L.; Min, X. A three-stage resilience analysis framework for urban infrastructure systems. Struct. Saf. 2012, 36-37, 23-31. [CrossRef]

23. Panteli, M.; Trakas, D.N.; Mancarella, P.; Hatziargyriou, N. Power Systems Resilience Assessment: Hardening and Smart Operational Enhancement Strategies. Proc. IEEE 2017, 105, 1202-1213. [CrossRef]

24. Bao, M.; Ding, Y.; Sang, M.; Li, D.; Shao, C.; Yan, J. Modeling and evaluating nodal resilience of multi-energy systems under windstorms. Appl. Energy 2020, 270, 115136. [CrossRef]

25. Cresta, M.; Gatta, F.; Geri, A.; Maccioni, M.; Paulucci, M. Resilience Assessment in Distribution Grids: A Complete Simulation Model. Energies 2021, 14, 4303. [CrossRef]

26. Bragatto, T.; Cresta, M.; Cortesi, F.; Gatta, F.M.; Geri, A.; Maccioni, M.; Paulucci, M. Assessment and Possible Solution to Increase Resilience: Flooding Threats in Terni Distribution Grid. Energies 2019, 12, 744. [CrossRef]

27. Brugnetti, E.; Coletta, G.; De Caro, F.; Vaccaro, A.; Villacci, D. Enabling Methodologies for Predictive Power System Resilience Analysis in the Presence of Extreme Wind Gusts. Energies 2020, 13, 3501. [CrossRef]

28. Mustafa, A.; Barabadi, A. Resilience Assessment of Wind Farms in the Arctic with the Application of Bayesian Networks. Energies 2021, 14, 4439. [CrossRef]

29. Mutani, G.; Todeschi, V.; Beltramino, S. Energy Consumption Models at Urban Scale to Measure Energy Resilience. Sustainability 2020, 12, 5678. [CrossRef]

30. Petit, F.; Phillips, J.; Verner, D. Resilience: Theory and Applications. In Decision and Information Sciences Division, Argonne National Laboratory. Available online: http:/ / www.dis.anl.gov/pubs/72218.Pdf (accessed on 1 February 2022).

31. Roege, P.E.; Collier, Z.A.; Mancillas, J.; McDonagh, J.A.; Linkov, I. Metrics for energy resilience. Energy Policy 2014, $72,249-256$. [CrossRef]

32. Orencio, P.M.; Fujii, M. A localized disaster-resilience index to assess coastal communities based on an analytic hierarchy process (AHP). Int. J. Disaster Risk Reduct. 2013, 3, 62-75. [CrossRef]

33. Watson, J.-P.; Guttromson, R.; Silva-Monroy, C.; Jeffers, R.; Jones, K.; Ellison, J.; Rath, C.; Gearhart, J.; Jones, D.; Corbet, T.; et al. Conceptual Framework for Developing Resilience Metrics for the Electricity, Oil, and Gas Sectors in the United States; Sandia National Labaratories: Albuquerque, NM, USA; Livermore, CA, USA, 2014.

34. Kharrazi, A.; Sato, M.; Yarime, M.; Nakayama, H.; Yu, Y.; Kraines, S. Examining the resilience of national energy systems: Measurements of diversity in production-based and consumption-based electricity in the globalization of trade networks. Energy Policy 2015, 87, 455-464. [CrossRef] 
35. Shannon, C.E. A mathematical theory of communication. Bell Syst. Tech. J. 1948, 27, 379-423. [CrossRef]

36. Simpson, E.H. Measurement of diversity. Nature 1949, 163, 688. [CrossRef]

37. Heino, O.; Takala, A.; Jukarainen, P.; Kalalahti, J.; Kekki, T.; Verho, P. Critical Infrastructures: The Operational Environment in Cases of Severe Disruption. Sustainability 2019, 11, 838. [CrossRef]

38. Páez-Curtidor, N.; Keilmann-Gondhalekar, D.; Drewes, J.E. Application of the Water-Energy-Food Nexus Approach to the Climate-Resilient Water Safety Plan of Leh Town, India. Sustainability 2021, 13, 10550. [CrossRef]

39. Kong, J.; Simonovic, S.P.; Zhang, C. Resilience Assessment of Interdependent Infrastructure Systems: A Case Study Based on Different Response Strategies. Sustainability 2019, 11, 6552. [CrossRef]

40. Chen, Z.; Liang, Y.; Jin, F. Simulation of city network accessibility and its influence on regional development pattern in China based on integrated land transport system. Prog. Geogr. 2021, 40, 183-193. [CrossRef]

41. Barreiro, J.; Lopes, R.; Ferreira, F.; Brito, R.; Telhado, M.J.; Matos, J.S.; Matos, R.S. Assessing Urban Resilience in Complex and Dynamic Systems: The RESCCUE Project Approach in Lisbon Research Site. Sustainability 2020, 12, 8931. [CrossRef]

42. Sharifi, A.; Roosta, M.; Javadpoor, M. Urban Form Resilience: A Comparative Analysis of Traditional, Semi-Planned, and Planned Neighborhoods in Shiraz, Iran. Urban Sci. 2021, 5, 18. [CrossRef]

43. Afgan, N.H. Sustainability Paradigm: Intelligent Energy System. Sustainability 2010, 2, 3812-3830. [CrossRef]

44. Pei, J.; Liu, W.; Han, L. Research on Evaluation Index System of Chinese City Safety Resilience Based on Delphi Method and Cloud Model. Int. J. Environ. Res. Public Health 2019, 16, 3802. [CrossRef]

45. Tan, L. Study on Comprehensive Risk Assessment of Urban Disaster in Xiamen from the Perspective of Resilience. In Proceedings of the Annual National Planning Conference 2019, Chonqing, China, 13-16 April 2019; p. 12.

46. Ouyang, X. Composite Risk Assessment of Urban Disaster; Jiangxi University of Science and Technology: Ganzhou, China, 2010.

47. Chen, L.; Huang, Y.-C.; Bai, R.-Z.; Chen, A. Regional disaster risk evaluation of China based on the universal risk model. Nat. Hazards 2017, 89, 647-660. [CrossRef]

48. Lawson, E.; Farmani, R.; Woodley, E.; Butler, D. A Resilient and Sustainable Water Sector: Barriers to the Operationalisation of Resilience. Sustainability 2020, 12, 1797. [CrossRef]

49. Willis, H.H.; Loa, K. Measuring the Resilience of Energy Distribution Systems; RAND Corporation: Santa Monica, CA, USA, 2015.

50. Erker, S.; Stangl, R.; Stoeglehner, G. Resilience in the light of energy crises-Part I: A framework to conceptualise regional energy resilience. J. Clean. Prod. 2017, 164, 420-433. [CrossRef]

51. Division Method of East, West, Middle, and Northeast Regions in National Bureau of Statistics of China. Available online: http:/ / www.stats.gov.cn (accessed on 1 February 2022).

52. McCarthy, R.W.; Ogden, J.M.; Sperling, D. Assessing reliability in energy supply systems. Energy Policy 2007, 35, $2151-2162$. [CrossRef]

53. Brancucci Martínez-Anido, C.; Bolado, R.; De Vries, L.; Fulli, G.; Vandenbergh, M.; Masera, M. European power grid reliability indicators, what do they really tell? Electr. Power Syst. Res. 2012, 90, 79-84. [CrossRef]

54. Bhatnagar, D.; Currier, A.; Hernandez, J.; Ma, O.; Kirby, B. Market and Policy Barriers to Energy Storage Deployment: A Study for the Energy Storage Systems Program; Sandia National Labaratories: Livermore, CA, USA, 2013.

55. Keogh, M.; Cody, C.; Grants, N. Resilience in Regulated Utilities; The National Association of Regulatory Utility Commissioners: Washington, DC, USA, 2013; p. 24.

56. Doukas, H.; Karakosta, C.; Flamos, A.; Psarras, J. Electric power transmission: An overview of associated burdens. Int. J. Energy Res. 2011, 35, 979-988. [CrossRef]

57. Lin, Y.; Bie, Z.; Qiu, A. A review of key strategies in realizing power system resilience. Glob. Energy Interconnect. 2018, 1, 70-78.

58. Dvorak, Z.; Chovancikova, N.; Bruk, J.; Hromada, M. Methodological Framework for Resilience Assessment of Electricity Infrastructure in Conditions of Slovak Republic. Int. J. Environ. Res. Public Health 2021, 18, 8286. [CrossRef]

59. Ward, D.M. The effect of weather on grid systems and the reliability of electricity supply. Clim. Chang. 2013, 121, 103-113. [CrossRef]

60. Bompard, E.; Napoli, R.; Xue, F. Extended topological approach for the assessment of structural vulnerability in transmission networks. IET Gener. Transm. Distrib. 2010, 4, 716-724. [CrossRef]

61. Willis, K.; Garrod, G. Electricity supply reliability: Estimating the value of lost load. Energy Policy 1997, 25, 97-103. [CrossRef]

62. Molyneaux, L.; Wagner, L.; Froome, C.; Foster, J. Resilience and electricity systems: A comparative analysis. Energy Policy 2012, 47, 188-201. [CrossRef]

63. Wang, Y.; Guo, C. The Study on Effect of Random Maintenance Quality of Components on Composite Power System Reliability. In Proceedings of the 2013 IEEE PES Asia-Pacific Power and Energy Engineering Conference (APPEEC), Hong Kong, China, 8-11 December 2013; pp. 1-6. [CrossRef]

64. Tao, S.; Hadjsaid, N.; Xiao, X.; Kieny, C. Power Quality \& Reliability Assessment of Distribution System Considering Voltage Interruptions and Sags. In Proceedings of the IEEE 15th International Conference on Harmonics and Quality of Power, Hong Kong, China, 17-20 June 2012; pp. 751-757.

65. Billinton, R.; Wangdee, W. Utilizing Bulk Electric System Reliability Performance Index Probability Distributions in a Performance Based Regulation Framework. In Proceedings of the 2006 International Conference on Probabilistic Methods Applied to Power Systems, Stocholm, Sweden, 11-15 June 2006; pp. 1-6. 
66. Voorspools, K.R.; D'Haeseleer, W.D. Reliability of power stations: Stochastic versus derated power approach. Int. J. Energy Res. 2003, 28, 117-129. [CrossRef]

67. Gnansounou, E. Assessing the energy vulnerability: Case of industrialised countries. Energy Policy 2008, 36, 3734-3744. [CrossRef]

68. Wang, H.; Lin, Z.; Wen, F.; Huang, J. A comprehensive evaluation index system for power system operation. In Proceedings of the International Conference on Sustainable Power Generation and Supply (SUPERGEN 2012), Hangzhou, China, 8-9 September 2012; pp. 1-6.

69. Pasimeni, M.R.; Petrosillo, I.; Aretano, R.; Semeraro, T.; De Marco, A.; Zaccarelli, N.; Zurlini, G. Scales, strategies and actions for effective energy planning: A review. Energy Policy 2014, 65, 165-174. [CrossRef]

70. Nathwani, J.; Chen, Z.; Case, M.P.; Collier, Z.A.; Roege, C.P.E.; Thorne, S.; Goldsmith, W.; Ragnarsdóttir, K.V.; Marks, P.M.; Ogrodowski, M. Sustainable Energy Pathways for Smart Urbanization and Off Grid Access: Options and Policies for Military Installations and Remote Communities. In Sustainable Cities and Military Installations; Linkov, I., Ed.; Springer: Dordrecht, The Netherlands, 2014; pp. 229-261.

71. Afgan, N.; Cvetinović, D. Wind power plant resilience. Therm. Sci. 2010, 14, 533-540. [CrossRef]

72. Fisher, R.E.; Bassett, G.W.; Buehring, W.A.; Collins, M.J.; Dickinson, D.C.; Eaton, L.K.; Haffenden, R.A.; Hussar, N.E.; Klett, M.S.; Lawlor, M.A.; et al. Constructing a Resilience Index for the Enhanced Critical Infrastructure Protection Program; Argonne National Lab (ANL): Chicago, IL, USA, 2010. Available online: https://www.osti.gov/servlets/purl/991101 (accessed on 1 February 2022). [CrossRef]

73. Afgan, N.; Cvetinovic, D. Resilience Evaluation of the Southeast European Natural Gas Routes System Catastrophe. Int. J. Eng. Innov. Technol. 2013, 3, 6 .

74. Eto, J.H.; Lacommare, K.H. Tracking the Reliability of the U.S. Electric Power System: An Assessment of Publicly Available Information Reported to State Public Utility Commissions; Lawrence Berkeley National Lab. (LBNL): Berkeley, CA, USA, 2008 ; p. 52.

75. Bagchi, A.; Sprintson, A.; Singh, C. Modeling the impact of fire spread on an electrical distribution network. Electr. Power Syst. Res. 2013, 100, 15-24. [CrossRef]

76. Roe, E.; Schulman, P.R. Toward a Comparative Framework for Measuring Resilience in Critical Infrastructure Systems. J. Comp. Policy Anal. Res. Pr. 2012, 14, 114-125. [CrossRef]

77. Carvalho, R.; Buzna, L.; Bono, F.; Masera, M.; Arrowsmith, D.K.; Helbing, D. Resilience of Natural Gas Networks during Conflicts, Crises and Disruptions. PLoS ONE 2014, 9, e90265. [CrossRef]

78. Poljanšek, K.; Bono, F.; Gutiérrez, E. Seismic risk assessment of interdependent critical infrastructure systems: The case of European gas and electricity networks. Earthq. Eng. Struct. Dyn. 2011, 41, 61-79. [CrossRef]

79. Nadeau, J. Improving the Resiliency of the Natural Gas Supply and Distribution Network; Naval Postgraduate School: Monterey, CA, USA, 2007.

80. Turnquist, M.; Vugrin, E. Design for resilience in infrastructure distribution networks. Environ. Syst. Decis. 2013, 33, 104-120. [CrossRef]

81. Ellison, J.F.; Corbet, T.F.; Brooks, R.E. Natural Gas Network Resiliency to a "Shakeout Scenario" Earthquake; Sandia National Laboratory: Albuquerque, NM, USA, 2013.

82. Hsu, B.-M.; Shu, M.-H.; Tsao, M. Reliability Measures for Liquefied Natural Gas Receiving Terminal Based on the Failure Information of Emergency Shutdown System; InTech: Shanghai, China, 2010.

83. Vugrin, E.D.; Warren, D.E.; Ehlen, M.A. A resilience assessment framework for infrastructure and economic systems: Quantitative and qualitative resilience analysis of petrochemical supply chains to a hurricane. Process Saf. Prog. 2011, 30, 280-290. [CrossRef]

84. Ellison, J.; Kelic, A.; Corbet, T.F. Is a Natural Gas Strategic Reserve for the US Necessary? A System Dynamics Approach; Sandia National Laboratory: Albuquerque, NM, USA, 2007.

85. Reymond, M. European key issues concerning natural gas: Dependence and vulnerability. Energy Policy 2007, 35, 4169-4176. [CrossRef]

86. Vera, I.; Langlois, L. Energy indicators for sustainable development. Energy 2007, 32, 875-882. [CrossRef]

87. Ang, B.; Choong, W.; Ng, A. A framework for evaluating Singapore's energy security. Appl. Energy 2015, 148, 314-325. [CrossRef]

88. Gracceva, F.; Zeniewski, P. A systemic approach to assessing energy security in a low-carbon EU energy system. Appl. Energy 2014, 123, 335-348. [CrossRef]

89. Månsson, A.; Johansson, B.; Nilsson, L.J. Assessing energy security: An overview of commonly used methodologies. Energy 2014, 73, 1-14. [CrossRef]

90. Skea, J.; Ekins, P.; Winskel, M. Energy 2050: Making the Transition to a Secure Low Carbon Energy System; Routledge: Oxfordshire, UK, 2011.

91. Martchamadol, J.; Kumar, S. Thailand's energy security indicators. Renew. Sustain. Energy Rev. 2012, 16, 6103-6122. [CrossRef]

92. Pachauri, S.; Spreng, D. Measuring and monitoring energy poverty. Energy Policy 2011, 39, 7497-7504. [CrossRef]

93. Pike, A.; Dawley, S.; Tomaney, J. Resilience, Adaptation and Adaptability. Camb. J. Reg. Econ. Soc. 2010, 3, 59-70. [CrossRef]

94. Kruyt, B.; van Vuuren, D.; de Vries, H.; Groenenberg, H. Indicators for energy security. Energy Policy 2009, 37, $2166-2181$. [CrossRef]

95. Newell, E.B.; Marsh, D.M.; Sharma, D. Enhancing the Resilience of the Australian National Electricity Market: Taking a Systems Approach in Policy Development. Ecol. Soc. 2011, 16, 15. [CrossRef] 
96. Farrell, A.E.; Zerriffi, H.; Dowlatabadi, H. Energy infrastructure and security. Annu. Rev. Environ. Resour. 2004, 29, 421-469. [CrossRef]

97. Wardekker, J.A.; de Jong, A.; Knoop, J.M.; van der Sluijs, J.P. Operationalising a resilience approach to adapting an urban delta to uncertain climate changes. Technol. Forecast. Soc. Chang. 2010, 77, 987-998. [CrossRef]

98. Ang, B.W.; Choong, W.L.; Ng, T.S. Energy security: Definitions, dimensions and indexes. Renew. Sustain. Energy Rev. 2015, 42, 1077-1093. [CrossRef]

99. Sovacool, B.K.; Mukherjee, I. Conceptualizing and measuring energy security: A synthesized approach. Energy 2011, 36, 5343-5355. [CrossRef]

100. Blum, H.; Legey, L.F. The challenging economics of energy security: Ensuring energy benefits in support to sustainable development. Energy Econ. 2012, 34, 1982-1989. [CrossRef]

101. Toka, A.; Iakovou, E.; Vlachos, D.; Tsolakis, N.; Grigoriadou, A.-L. Managing the diffusion of biomass in the residential energy sector: An illustrative real-world case study. Appl. Energy 2014, 129, 56-69. [CrossRef]

102. Hoggett, R. Technology scale and supply chains in a secure, affordable and low carbon energy transition. Appl. Energy 2014, 123, 296-306. [CrossRef]

103. Dassisti, M.; Carnimeo, L. A small-world methodology of analysis of interchange energy-networks: The European behaviour in the economic crisis. Energy Policy 2013, 63, 887-899. [CrossRef]

104. Agudelo-Vera, C.M.; Leduc, W.R.; Mels, A.R.; Rijnaarts, H.H. Harvesting urban resources towards more resilient cities Resour. Conserv. Recycl. 2012, 64, 3-12. [CrossRef]

105. Opitz-Stapleton, S.; Seraydarian, L.; MacClune, K.; Guibert, G.; Reed, S.; Uennatornwaranggoon, F.; del Rio, C.R. Building Resilience to Climate Change in Asian Cities. In Resilient Cities, Dordrecht; Otto-Zimmermann, K., Ed.; Springer: Dordrecht, The Netherlands, 2011; pp. 401-409.

106. Beck, M.B.; Villarroel Walker, R. Nexus security: Governance, innovation and the resilient city. Front. Environ. Sci. Eng. 2013, 7, 640-657. [CrossRef]

107. Matthews, E.C.; Sattler, M.; Friedland, C.J. A critical analysis of hazard resilience measures within sustainability assessment frameworks. Environ. Impact Assess. Rev. 2014, 49, 59-69. [CrossRef]

108. McLellan, B.; Zhang, Q.; Farzaneh, H.; Utama, N.A.; Ishihara, K.N. Resilience, Sustainability and Risk Management: A Focus on Energy. Challenges 2012, 3, 153-182. [CrossRef]

109. Esteban, M.; Portugal-Pereira, J. Post-disaster resilience of a 100\% renewable energy system in Japan. Energy 2014, 68, 756-764. [CrossRef]

110. Mazur, C.; Hoegerle, Y.; Brucoli, M.; van Dam, K.; Guo, M.; Markides, C.; Shah, N. A holistic resilience framework development for rural power systems in emerging economies. Appl. Energy 2018, 235, 219-232. [CrossRef]

111. De Castro, D.; Kim, A. Adaptive or Absent: A Critical Review of Building System Resilience in the LEED Rating System. Sustainability 2021, 13, 6697. [CrossRef]

112. Exner, A.; Politti, E.; Schriefl, E.; Erker, S.; Stangl, R.; Baud, S.; Warmuth, H.; Matzenberger, J.; Kranzl, L.; Paulesich, R.; et al. Measuring regional resilience towards fossil fuel supply constraints. Adaptability and vulnerability in socio-ecological Transformations-the case of Austria. Energy Policy 2016, 91, 128-137. [CrossRef]

113. McGuirk, P.; Dowling, R.; Bulkeley, H. Repositioning urban governments? Energy efficiency and Australia's changing climate and energy governance regimes. Urban Stud. 2014, 51, 2717-2734. [CrossRef]

114. Gatto, A.; Drago, C. Measuring and modeling energy resilience. Ecol. Econ. 2020, 172, 106527. [CrossRef]

115. Kennedy, C.; Corfee-Morlot, J. Past performance and future needs for low carbon climate resilient infrastructure-An investment perspective. Energy Policy 2013, 59, 773-783. [CrossRef]

116. Scott, C.A.; Pierce, S.A.; Pasqualetti, M.J.; Jones, A.L.; Montz, B.E.; Hoover, J.H. Policy and institutional dimensions of the water-energy nexus. Energy Policy 2011, 39, 6622-6630. [CrossRef]

117. van Renssen, S. Energy security vs climate policy. Nat. Clim. Change 2014, 4, 756-757. [CrossRef]

118. Bruneau, M.; Chang, S.E.; Eguchi, R.T.; Lee, G.C.; O’Rourke, T.D.; Reinhorn, A.M.; Shinozuka, M.; Tierney, K.; Wallace, W.A.; Von Winterfeldt, D. A Framework to Quantitatively Assess and Enhance the Seismic Resilience of Communities. Earthq. Spectra 2003, 19, 733-752. [CrossRef]

119. Gautam, P.; Piya, P.; Karki, R. Resilience Assessment of Distribution Systems Integrated with Distributed Energy Resources. IEEE Trans. Sustain. Energy 2020, 12, 338-348. [CrossRef]

120. Grafakos, S.; Enseñado, E.M.; Flamos, A. Developing an integrated sustainability and resilience framework of indicators for the assessment of low-carbon energy technologies at the local level. Int. J. Sustain. Energy 2016, 36, 945-971. [CrossRef]

121. Sharifi, A.; Yamagata, Y. Major principles and criteria for development of an urban resilience assessment index. In Proceedings of the International Conference and Utility Exhibition 2014 on Green Energy for Sustainable Development (ICUE 2014), Pattaya City, Thailand, 19-21 March 2014; p. 5.

122. Charoenkit, S.; Kumar, S. Environmental sustainability assessment tools for low carbon and climate resilient low income housing settlements. Renew. Sustain. Energy Rev. 2014, 38, 509-525. [CrossRef]

123. Flynn, S.; Burke, S. Powering America's Energy Resilience; Center for National Policy: Washington, DC, USA, 2012.

124. Hay, A.H. Surviving catastrophic events: Stimulating community resilience. In Infrastructure Risk and Resilience: Transportation, Institution of Engineering and Technology; IET: London, UK, 2013; pp. 41-46. [CrossRef] 
125. Thomas, S.; Kerner, D. Defense Energy Resilience: Lessons from Ecology; U.S. Army War College, Strategic Studies Institute: Carlisle, PA, USA, 2010; p. 53.

126. PEER: Performance Excellence in Electricity Renewal. Standard-Criteria Document Detailing Metrics Included in the Performance Category: Reliability, Power Quality, and Safety; Perfect Power Institute: Wexford, PA, USA, 2013.

127. Ko, Y.; Barrett, B.F.D.; Copping, A.E.; Sharifi, A.; Yarime, M.; Wang, X. Energy Transitions Towards Low Carbon Resilience: Evaluation of Disaster-Triggered Local and Regional Cases. Sustainability 2019, 11, 6801. [CrossRef]

128. National Infrastructure Advisory Council. Strengthening Regional Resilience through National, Regional, and Sector Partnerships; DRAFT Report and Recommendations; National Infrastructure Advisory Council: Washington, DC, USA, 2013.

129. U.S.-Canada Power System Outage Task Force. Final Report on the 14 August 2003 Blackout in the United States and Canada: Causes and Recommendations; U.S.-Canada Power System Outage Task Force: Washington, DC, USA, 2004; p. 238. Available online: http: / / cybercemetery.unt.edu/archive/energyreport/20090701154100/https:/ / reports.energy.gov/ (accessed on 1 February 2022).

130. Holling, C. Engineering resilience versus ecological resilience. Eng. Ecol. Constraints 1996, 31, 32.

131. Walker, B.; Holling, C.S.; Carpenter, S.R.; Kinzig, A. Resilience, Adaptability and Transformability in Social-ecological Systems. Ecol. Soc. 2004, 9, 5. [CrossRef]

132. Cutter, S.L.; Burton, C.G.; Emrich, C.T. Disaster Resilience Indicators for Benchmarking Baseline Conditions. J. Homel. Secur. Emerg. Manag. 2010, 7, 1-22. [CrossRef]

133. Linkov, I.; Eisenberg, D.A.; Plourde, K.; Seager, T.P.; Allen, J.; Kott, A. Resilience metrics for cyber systems. Environ. Syst. Decis. 2013, 33, 471-476. [CrossRef]

134. Cutter, S.L.; Barnes, L.; Berry, M.; Burton, C.; Evans, E.; Tate, E.; Webb, J. A place-based model for understanding community resilience to natural disasters. Glob. Environ. Chang. 2008, 18, 598-606. [CrossRef]

135. Campanella, T.J. Urban Resilience and the Recovery of New Orleans. J. Am. Plan. Assoc. 2006, 72, 141-146. [CrossRef]

136. Dodson, J. Suburbia under an Energy Transition: A Socio-technical Perspective. Urban Stud. 2013, 51, 1487-1505. [CrossRef]

137. Shaw, A.; Burch, S.; Kristensen, F.; Robinson, J.; Dale, A. Accelerating the sustainability transition: Exploring synergies between adaptation and mitigation in British Columbian communities. Glob. Environ. Chang. 2014, 25, 41-51. [CrossRef]

138. Minne, L.; Pandit, A.; Crittenden, J.C.; Begovic, M.M.; Kim, I.; Jeong, H.; James, J.-A.; Lu, Z.; Xu, M.; French, S.; et al. Energy and Water Interdependence, and Their Implications for Urban Areas. In Encyclopedia of Sustainability Science and Technology; Meyers, R.A., Ed.; Springer: New York, NY, USA, 2012; pp. 3449-3471.

139. Lee, T.; Lee, T.; Lee, Y. An experiment for urban energy autonomy in Seoul: The One 'Less' Nuclear Power Plant policy. Energy Policy 2014, 74, 311-318. [CrossRef]

140. Pederson, P.; Dudenhoeffer, D.; Hartley, S.; Permann, M. Critical Infrastructure Interdependency Modeling: A Survey of U.S. and International Research; Idaho National Lab (INL): Idaho Falls, ID, USA, 2006. Available online: https://www.osti.gov/servlets/ purl/911792 (accessed on 1 February 2022). [CrossRef]

141. Rutherford, J.; Coutard, O. Urban Energy Transitions: Places, Processes and Politics of Socio-technical Change. Urban Stud. 2014, 51, 1353-1377. [CrossRef]

142. Bristow, D.N.; Kennedy, C.A. Urban Metabolism and the Energy Stored in Cities. J. Ind. Ecol. 2013, 17, 656-667. [CrossRef]

143. Mulugetta, Y.; Urban, F. Deliberating on low carbon development. Energy Policy 2010, 38, 7546-7549. [CrossRef]

144. Esteban, M.; Zhang, Q.; Utama, A. Estimation of the energy storage requirement of a future $100 \%$ renewable energy system in Japan. Energy Policy 2012, 47, 22-31. [CrossRef]

145. Coaffee, J. Risk, resilience, and environmentally sustainable cities. Energy Policy 2008, 36, 4633-4638. [CrossRef]

146. Ritchie, H.; Hardy, M.; Lloyd, M.G.; McGreal, S. Big Pylons: Mixed signals for transmission. Spatial planning for energy distribution. Energy Policy 2013, 63, 311-320. [CrossRef]

147. Bahaj, A.; James, P. Urban energy generation: The added value of photovoltaics in social housing. Renew. Sustain. Energy Rev. 2007, 11, 2121-2136. [CrossRef]

148. Marique, A.-F.; Reiter, S. A simplified framework to assess the feasibility of zero-energy at the neighbourhood/community scale. Energy Build. 2014, 82, 114-122. [CrossRef]

149. Lloyd-Jones, T. Retrofitting sustainability to historic city core areas. Proc. Inst. Civ. Eng. Munic. Eng. 2010, 163, 179-188. [CrossRef]

150. Manfren, M.; Caputo, P.; Costa, G. Paradigm shift in urban energy systems through distributed generation: Methods and models. Appl. Energy 2011, 88, 1032-1048. [CrossRef]

151. Martin, C.; Taylor, P.G.; Upham, P.; Ghiasi, G.; Bale, C.S.; James, H.; Owen, A.; Gale, W.F.; Slack, R.J.; Helmer, S. Energy in low carbon cities and social learning: A process for defining priority research questions with UK stakeholders. Sustain. Cities Soc. 2014, 10, 149-160. [CrossRef]

152. Van den Dobbelsteen, A.A.J.F.; Keeffe, G.; Tillie, N.M.J.D. Cities ready for energy crisis: Building urban energy resilience. In Proceedings of the SASBE 2012: 4th CIB International Conference on Smart and Sustainable Built Environments; Sao Paulo, Brasil, 27-30 June 2012, CIB International Council for Research and Innovation in Building and Construction: Ottawa, ON, Canada; pp. 1-10.

153. Akbari, H.; Matthews, H.D. Global cooling updates: Reflective roofs and pavements. Energy Build. 2012, 55, 2-6. [CrossRef]

154. Gleeson, B. Critical Commentary. Waking from the Dream: An Australian Perspective on Urban Resilience. Urban Stud. 2008, 45, 2653-2668. [CrossRef]

155. Hussain, A.; Bui, V.-H.; Kim, H.-M. Microgrids as a resilience resource and strategies used by microgrids for enhancing resilience. Appl. Energy 2019, 240, 56-72. [CrossRef] 
156. Li, Z.; Shahidehpour, M.; Aminifar, F.; AlAbdulwahab, A.; Al-Turki, Y. Networked Microgrids for Enhancing the Power System Resilience. Proc. IEEE 2017, 105, 1289-1310. [CrossRef]

157. Moss, T. Divided City, Divided Infrastructures: Securing Energy and Water Services in Postwar Berlin. J. Urban Hist. 2009, 35, 923-942. [CrossRef]

158. Arghandeh, R.; Brown, M.; Del Rosso, A.; Ghatikar, G.; Stewart, E.; Vojdani, A.; Von Meier, A. The Local Team: Leveraging Distributed Resources to Improve Resilience. IEEE Power Energy Mag. 2014, 12, 76-83. [CrossRef]

159. NIST. Disaster Resilience Framework, 75\% Draft for San Diego, CA Workshop; National Institute of Standards and Technology: Gaithersburg, MD, USA, 2015.

160. Linkov, I.; Bridges, T.S.; Creutzig, F.; Decker, J.; Fox-Lent, C.; Kröger, W.; Lambert, J.H.; Levermann, A.; Montreuil, B.; Nathwani, J.; et al. Changing the resilience paradigm. Nat. Clim. Chang. 2014, 4, 407-409. [CrossRef]

161. Nostrand, J. Keeping the Lights on During Superstorm Sandy: Climate Change Adaptation and the Resiliency Benefits of Distributed Generation. SSRN Electron. J. 2015, 23, 63. [CrossRef]

162. Warren, C.R.; McFadyen, M. Does community ownership affect public attitudes to wind energy? A case study from south-west Scotland. Land Use Policy 2010, 27, 204-213. [CrossRef]

163. Miller, W. What does build environment research have to do with risk mitigation, resilience and disaster recovery? Sustain. Cities Soc. 2015, 19, 91-97. [CrossRef]

164. Grubler, A.; Bai, X.; Buettner, T.; Dhakal, S.; Fisk, D.; Ichinose, T. Urban Energy Systems. In Global Energy Assessment-Toward a Sustainable Future; Cambridge University Press: Cambridge, UK; New York, NY, USA; International Institute for Applied Systems Analysis: Laxenburg, Austria, 2012; pp. 1307-1400.

165. O'Brien, G. Vulnerability and Resilience in the European Energy System. Energy Environ. 2009, 20, 399-410. [CrossRef]

166. Jabareen, Y. Planning the resilient city: Concepts and strategies for coping with climate change and environmental risk. Cities 2013, 31, 220-229. [CrossRef]

167. Adams, C.; Bell, S. Local energy generation projects: Assessing equity and risks. Local Environ. 2014, 20, 1473-1488. [CrossRef]

168. Caputo, S.; Caserio, M.; Coles, R.; Jankovic, L.; Gaterell, M.R. Testing energy efficiency in urban regeneration. Proc. Inst. Civ. Eng. Eng. Sustain. 2012, 165, 69-80. [CrossRef]

169. Collier, M.J.; Nedović-Budić, Z.; Aerts, J.; Connop, S.; Foley, D.; Foley, K.; Newport, D.; McQuaid, S.; Slaev, A.D.; Verburg, P. Transitioning to resilience and sustainability in urban communities. Cities 2013, 32, S21-S28. [CrossRef]

170. Bourdic, L.; Salat, S.; Nowacki, C. Assessing cities: A new system of cross-scale spatial indicators. Build. Res. Inf. 2012, 40, 592-605. [CrossRef]

171. Broto, V.C.; Bulkeley, H. A survey of urban climate change experiments in 100 cities. Glob. Environ. Chang. 2012, $23,92-102$. [CrossRef] [PubMed]

172. Xiao, L.; Li, X.; Wang, R. Integrating climate change adaptation and mitigation into sustainable development planning for Lijiang City. Int. J. Sustain. Dev. World Ecol. 2011, 18, 515-522. [CrossRef]

173. Wei, T.; Tang, Z. Building low-carbon cities: Assessing the fast growing U.S. cities' land use comprehensive plans. J. Environ. Assess. Policy Manag. 2014, 16, 1450003. [CrossRef]

174. Su, M.; Fath, B.D. Spatial distribution of urban ecosystem health in Guangzhou, China. Ecol. Indic. 2012, 15, 122-130. [CrossRef]

175. Schuetze, T.; Lee, J.-W.; Lee, T.-G. Sustainable Urban (re-)Development with Building Integrated Energy, Water and Waste Systems. Sustainability 2013, 5, 1114-1127. [CrossRef]

176. Mourshed, M. The impact of the projected changes in temperature on heating and cooling requirements in buildings in Dhaka, Bangladesh. Appl. Energy 2011, 88, 3737-3746. [CrossRef]

177. Mittal, V.; Kasana, K.; Thakur, N. The study of solar absorption air-conditioning systems. J. Energy South. Afr. 2005, 16, 59-66. [CrossRef]

178. Georgiadou, M.-C.; Hacking, T. Future-Proofed Design for Sustainable Communities. In Sustainability in Energy and Buildings; Howlett, R.J., Jain, L.C., Lee, S.H., Eds.; Springer: Berlin/Heidelberg, Germany, 2011; pp. 179-188.

179. McIntyre, J.H.; Lubitz, W.D.; Stiver, W.H. Local wind-energy potential for the city of Guelph, Ontario (Canada). Renew. Energy 2011, 36, 1437-1446. [CrossRef]

180. Temby, O.; Kapsis, K.; Berton, H.; Rosenbloom, D.; Gibson, G.; Athienitis, A.; Meadowcroft, J. Building-Integrated Photovoltaics: Distributed Energy Development for Urban Sustainability. Environ. Sci. Policy Sustain. Dev. 2014, 56, 4-17. [CrossRef]

181. Woo, C.; Li, R.; Shiu, A.; Horowitz, I. Residential winter kWh responsiveness under optional time-varying pricing in British Columbia. Appl. Energy 2013, 108, 288-297. [CrossRef]

182. Hodbod, J.; Adger, W.N. Integrating social-ecological dynamics and resilience into energy systems research. Energy Res. Soc. Sci. 2014, 1, 226-231. [CrossRef]

183. Mulyono, N.B. Mutual Support in Energy Sector: Toward Energy Resilience. Procedia Comput. Sci. 2015, 60, 1041-1050. [CrossRef]

184. Rezaie, B.; Rosen, M.A. District heating and cooling: Review of technology and potential enhancements. Appl. Energy 2011, 93, 2-10. [CrossRef]

185. Spinney, J. Sustainable Home Refurbishment: The Earthscan Expert Guide to Retrofitting Homes for Efficiency. Hous. Stud. 2011, 26, 800-802. [CrossRef]

186. Brand, U.; Von Gleich, A. Transformation toward a Secure and Precaution-Oriented Energy System with the Guiding Concept of Resilience-Implementation of Low-Exergy Solutions in Northwestern Germany. Energies 2015, 8, 6995-7019. [CrossRef] 
187. Perrone, D.; Hornberger, G.M. Water, food, and energy security: Scrambling for resources or solutions? WIREs Water 2014, 1, 49-68. [CrossRef]

188. Sage, C. The interconnected challenges for food security from a food regimes perspective: Energy, climate and malconsumption. J. Rural Stud. 2013, 29, 71-80. [CrossRef] 
189. Khan, S.; Hanjra, M.A. Footprints of water and energy inputs in food production-Global perspectives. Food Policy 2009, 34, 130-140. [CrossRef]

190. Saha, M.; Eckelman, M.J. Geospatial assessment of potential bioenergy crop production on urban marginal land. Appl. Energy 2015, 159, 540-547. [CrossRef]

191. Byrd, H.; Matthewman, S. Exergy and the City: The Technology and Sociology of Power (Failure). J. Urban Technol. 2014, 21, 85-102. [CrossRef]

192. Stillwell, A.S.; King, C.W.; Webber, M.E.; Duncan, I.J.; Hardberger, A. The Energy-Water Nexus in Texas. Ecol. Soc. $2011,16$. [CrossRef]

193. Maliszewski, P.J.; Perrings, C. Factors in the resilience of electrical power distribution infrastructures. Appl. Geogr. 2012, 32, 668-679. [CrossRef]

194. Majithia, S. Improving Resilience Challenges and Linkages of the Energy Industry in Changing Climate. In Weather Matters for Energy; Troccoli, A., Dubus, L., Haupt, S.E., Eds.; Springer: New York, NY, USA, 2014; pp. 113-131.

195. Oliver-Solà, J.; Gabarrell, X.; Rieradevall, J. Environmental impacts of natural gas distribution networks within urban neighborhoods. Appl. Energy 2009, 86, 1915-1924. [CrossRef]

196. Bragdon, C.R.; Hronszky, I.; Nelson, G.L. Resilient Communities: From Sustainable to Secure. AIP Conf. Proc. 2009, 1157, 184-200. [CrossRef]

197. Begovic, M.M. Electrical Transmission Systems and Smart Grids-Selected Entries from the Encyclopedia of Sustainability Science and Technology; Springer: New York, NY, USA, 2012.

198. Cuadra, L.; Salcedo-Sanz, S.; Del Ser, J.; Jiménez-Fernández, S.; Geem, Z.W. A Critical Review of Robustness in Power Grids Using Complex Networks Concepts. Energies 2015, 8, 9211-9265. [CrossRef]

199. Bouffard, F.; Kirschen, D.S. Centralised and distributed electricity systems. Energy Policy 2008, 36, 4504-4508. [CrossRef]

200. Portugal Pereira, J.; Troncoso Parady, G.; Castro Dominguez, B. Japan's energy conundrum: Post-Fukushima scenarios from a life cycle perspective. Energy Policy 2014, 67, 104-115. [CrossRef]

201. Sovacool, B.K. Evaluating energy security in the Asia pacific: Towards a more comprehensive approach. Energy Policy 2011, 39, 7472-7479. [CrossRef]

202. Molyneaux, L.; Brown, C.; Foster, J.; Wagner, L. Measuring Resilience to Energy Shocks; MPRA Paper: Munich, Germany, 2015.

203. Yamagata, Y.; Seya, H. Spatial electricity sharing system for making city more resilient against X-Events. Innov. Supply Chain Manag. 2013, 7, 75-82. [CrossRef]

204. Lucey, W.P.; Barraclough, C.L.; Buchanan, S.E. Closed-Loop Water and Energy Systems: Implementing Nature's Design in Cities of the Future. In Water Infrastructure for Sustainable Communities: China and The World; Hao, X., Novotny, V., Nelson, V., Eds.; IWA: London, UK, 2010; p. 12.

205. Hubbart, J.A.; Kellner, E.; Hooper, L.; Lupo, A.R.; Market, P.S.; Guinan, P.E.; Stephan, K.; Fox, N.I.; Svoma, B.M. Localized Climate and Surface Energy Flux Alterations across an Urban Gradient in the Central U.S. Energies 2014, 7, 1770-1791. [CrossRef]

206. Stone, B.; Hess Jeremy, J.; Frumkin, H. Urban Form and Extreme Heat Events: Are Sprawling Cities More Vulnerable to Climate Change Than Compact Cities? Environ. Health Perspect. 2010, 118, 1425-1428. [CrossRef] [PubMed]

207. Gill, S.E.; Handley, J.F.; Ennos, A.R.; Pauleit, S. Adapting Cities for Climate Change: The Role of the Green Infrastructure. Built Environ. 2007, 33, 115-133. [CrossRef]

208. Cavan, G.; Lindley, S.; Jalayer, F.; Yeshitela, K.; Pauleit, S.; Renner, F.; Gill, S.; Capuano, P.; Nebebe, A.; Woldegerima, T.; et al. Urban morphological determinants of temperature regulating ecosystem services in two African cities. Ecol. Indic. 2014, 42, 43-57. [CrossRef]

209. Voskamp, I.; Van de Ven, F. Planning support system for climate adaptation: Composing effective sets of blue-green measures to reduce urban vulnerability to extreme weather events. Build. Environ. 2015, 83, 159-167. [CrossRef]

210. Akbari, H.; Pomerantz, M.; Taha, H. Cool surfaces and shade trees to reduce energy use and improve air quality in urban areas. Sol. Energy 2001, 70, 295-310. [CrossRef]

211. Akbari, H.; Rose, L.S. Urban Surfaces and Heat Island Mitigation Potentials. J. Hum.-Environ. Syst. 2008, 11, 85-101. [CrossRef]

212. Bretz, S.; Akbari, H.; Rosenfeld, A. Practical issues for using solar-reflective materials to mitigate urban heat islands. Atmos. Environ. 1998, 32, 95-101. [CrossRef]

213. Ong, B.L. Green plot ratio: An ecological measure for architecture and urban planning. Landsc. Urban Plan. 2003, 63, 197-211. [CrossRef]

214. Gómez-Muñoz, V.M.; Porta-Gándara, M.A.; Fernández, J.L. Effect of tree shades in urban planning in hot-arid climatic regions. Landsc. Urban Plan. 2010, 94, 149-157. [CrossRef]

215. House-Peters, L.A.; Chang, H. Modeling the impact of land use and climate change on neighborhood-scale evaporation and nighttime cooling: A surface energy balance approach. Landsc. Urban Plan. 2011, 103, 139-155. [CrossRef]

216. Millward, A.A.; Torchia, M.; Laursen, A.E.; Rothman, L.D. Vegetation Placement for Summer Built Surface Temperature Moderation in an Urban Microclimate. Environ. Manag. 2014, 53, 1043-1057. [CrossRef] [PubMed]

217. Bahadori, M.N. Passive Cooling Systems in Iranian Architecture. Sci. Am. 1978, 238, 144-154. [CrossRef]

218. Jamei, E.; Rajagopalan, P.; Seyedmahmoudian, M.; Jamei, Y. Review on the impact of urban geometry and pedestrian level greening on outdoor thermal comfort. Renew. Sustain. Energy Rev. 2016, 54, 1002-1017. [CrossRef] 
219. Novotny, V. Water-energy nexus: Retrofitting urban areas to achieve zero pollution. Build. Res. Inf. 2013, 41, 589-604. [CrossRef]

220. Chatterton, P. Towards an Agenda for Post-carbon Cities: Lessons from Lilac, the UK's First Ecological, Affordable Cohousing Community. Int. J. Urban Reg. Res. 2013, 37, 1654-1674. [CrossRef]

221. Malys, L.; Musy, M.; Inard, C. A hydrothermal model to assess the impact of green walls on urban microclimate and building energy consumption. Build. Environ. 2014, 73, 187-197. [CrossRef]

222. Alexandri, E.; Jones, P. Temperature decreases in an urban canyon due to green walls and green roofs in diverse climates. Build. Environ. 2008, 43, 480-493. [CrossRef]

223. Kikegawa, Y.; Genchi, Y.; Kondo, H.; Hanaki, K. Impacts of city-block-scale countermeasures against urban heat-island phenomena upon a building's energy-consumption for air-conditioning. Appl. Energy 2006, 83, 649-668. [CrossRef]

224. La Roche, P.; Berardi, U. Comfort and energy savings with active green roofs. Energy Build. 2014, 82, 492-504. [CrossRef]

225. Razzaghmanesh, M.; Beecham, S.; Brien, C. Developing resilient green roofs in a dry climate. Sci. Total Environ. 2014, 490, 579-589. [CrossRef] [PubMed]

226. Berardi, U.; GhaffarianHoseini, A.; GhaffarianHoseini, A. State-of-the-art analysis of the environmental benefits of green roofs. Appl. Energy 2014, 115, 411-428. [CrossRef]

227. Santamouris, M. Cooling the cities-A review of reflective and green roof mitigation technologies to fight heat island and improve comfort in urban environments. Sol. Energy 2012, 103, 682-703. [CrossRef]

228. Vieira, A.S.; Beal, C.; Ghisi, E.; Stewart, R. Energy intensity of rainwater harvesting systems: A review. Renew. Sustain. Energy Rev. 2014, 34, 225-242. [CrossRef]

229. Hussey, K.; Pittock, J. The Energy-Water Nexus: Managing the Links between Energy and Water for a Sustainable Future. Ecol. Soc. 2012, 17, 31. [CrossRef]

230. Dolman, N.; Savage, A.; Ogunyoye, F. Water-sensitive urban design: Learning from experience. Proc. Inst. Civ. Eng. Munic. Eng. 2013, 166, 86-97. [CrossRef]

231. Pisello, A.L.; Pignatta, G.; Castaldo, V.L.; Cotana, F. Experimental Analysis of Natural Gravel Covering as Cool Roofing and Cool Pavement. Sustainability 2014, 6, 4706-4722. [CrossRef]

232. Bouzarovski, S. Energy poverty in the European Union: Landscapes of vulnerability. WIREs Energy Environ. 2013, 3, 276-289. [CrossRef]

233. Jim, C. Air-conditioning energy consumption due to green roofs with different building thermal insulation. Appl. Energy 2014, 128, 49-59. [CrossRef]

234. Atkinson, J.G.; Jackson, T.; Mullings-Smith, E. Market influence on the low carbon energy refurbishment of existing multiresidential buildings. Energy Policy 2009, 37, 2582-2593. [CrossRef]

235. McKenna, R.; Merkel, E.; Fehrenbach, D.; Mehne, S.; Fichtner, W. Energy efficiency in the German residential sector: A bottom-up building-stock-model-based analysis in the context of energy-political targets. Build. Environ. 2013, 62, 77-88. [CrossRef]

236. Cole, R.J.; Fedoruk, L. Shifting from net-zero to net-positive energy buildings. Build. Res. Inf. 2014, 43, 111-120. [CrossRef]

237. Shimoda, Y.; Asahi, T.; Taniguchi, A.; Mizuno, M. Evaluation of city-scale impact of residential energy conservation measures using the detailed end-use simulation model. Energy 2007, 32, 1617-1633. [CrossRef]

238. Berdahl, P.; Bretz, S.E. Preliminary survey of the solar reflectance of cool roofing materials. Energy Build. 1997, 25, 149-158. [CrossRef]

239. Banfill, P.F.G.; Peacock, A.D. Energy-efficient new housing-The UK reaches for sustainability. Build. Res. Inf. 2007, 35, 426-436. [CrossRef]

240. Rendall, S.; Page, S.; Reitsma, F.; Van Houten, E.; Krumdieck, S. Quantifying Transport Energy Resilience: Active Mode Accessibility. Transp. Res. Rec. 2011, 2242, 72-80. [CrossRef]

241. Mindali, O.; Raveh, A.; Salomon, I. Urban density and energy consumption: A new look at old statistics. Transp. Res. Part A Policy Pr. 2004, 38, 143-162. [CrossRef]

242. Unger, N.; Bond, T.C.; Wang, J.S.; Koch, D.M.; Menon, S.; Shindell, D.T.; Bauer, S. Attribution of climate forcing to economic sectors. Proc. Natl. Acad. Sci. USA 2010, 107, 3382. [CrossRef]

243. Marshall, J.D. Energy-Efficient Urban Form. Environ. Sci. Technol. 2008, 42, 3133-3137. [CrossRef] [PubMed]

244. Luederitz, C.; Lang, D.J.; Von Wehrden, H. A systematic review of guiding principles for sustainable urban neighborhood development. Landsc. Urban Plan. 2013, 118, 40-52. [CrossRef]

245. Abdallah, K.B.; Belloumi, M.; De Wolf, D. Indicators for sustainable energy development: A multivariate cointegration and causality analysis from Tunisian road transport sector. Renew. Sustain. Energy Rev. 2013, 25, 34-43. [CrossRef]

246. Scanlon, B.R.; Duncan, I.; Reedy, R.C. Drought and the water-energy nexus in Texas. Environ. Res. Lett. 2013, 8, 045033. [CrossRef]

247. Perrone, D.; Murphy, J.; Hornberger, G.M. Gaining Perspective on the Water-Energy Nexus at the Community Scale. Environ. Sci. Technol. 2011, 45, 4228-4234. [CrossRef]

248. Webb, J. Enabling Urban Energy: Governance of Innovation in Two UK Cities. In Beyond the Networked City; Routledge: Oxfordshire, UK, 2015; p. 23.

249. Mashima, D.; Cárdenas, A.A. Evaluating Electricity Theft Detectors in Smart Grid Networks, Research in Attacks, Intrusions, and Defenses; Balzarotti, D., Stolfo, S.J., Cova, M., Eds.; Springer: Berlin/Heidelberg, Germany, 2012; pp. 210-229. 
250. Shih Chung, Y.; Scown Corinne, D.; Soibelman, L.; Matthews, H.S.; Garrett James, H.; Dodrill, K.; McSurdy, S. Data Management for Geospatial Vulnerability Assessment of Interdependencies in U.S. Power Generation. J. Infrastruct. Syst. 2009, 15, 179-189. [CrossRef]

251. Li, Y.; Qu, G. Urban disaster resilience evaluation and improvement strategies in China. Planners $2017,8,7$.

252. Milman, A.; Short, A. Incorporating resilience into sustainability indicators: An example for the urban water sector. Milman, A.; Short, A. Incorporating resilience into sustainability indicators: An example for the urban water sector. Glob. Environ. Chang. 2008, 18, 758-767. [CrossRef]

253. Rifat, S.; Liu, W. Measuring Community Disaster Resilience in the Conterminous Coastal United States. ISPRS Int. J. Geo Inf. 2020, 9, 469. [CrossRef]

254. Brunetta, G.; Salata, S. Mapping Urban Resilience for Spatial Planning-A First Attempt to Measure the Vulnerability of the System. Sustainability 2019, 11, 2331. [CrossRef] 